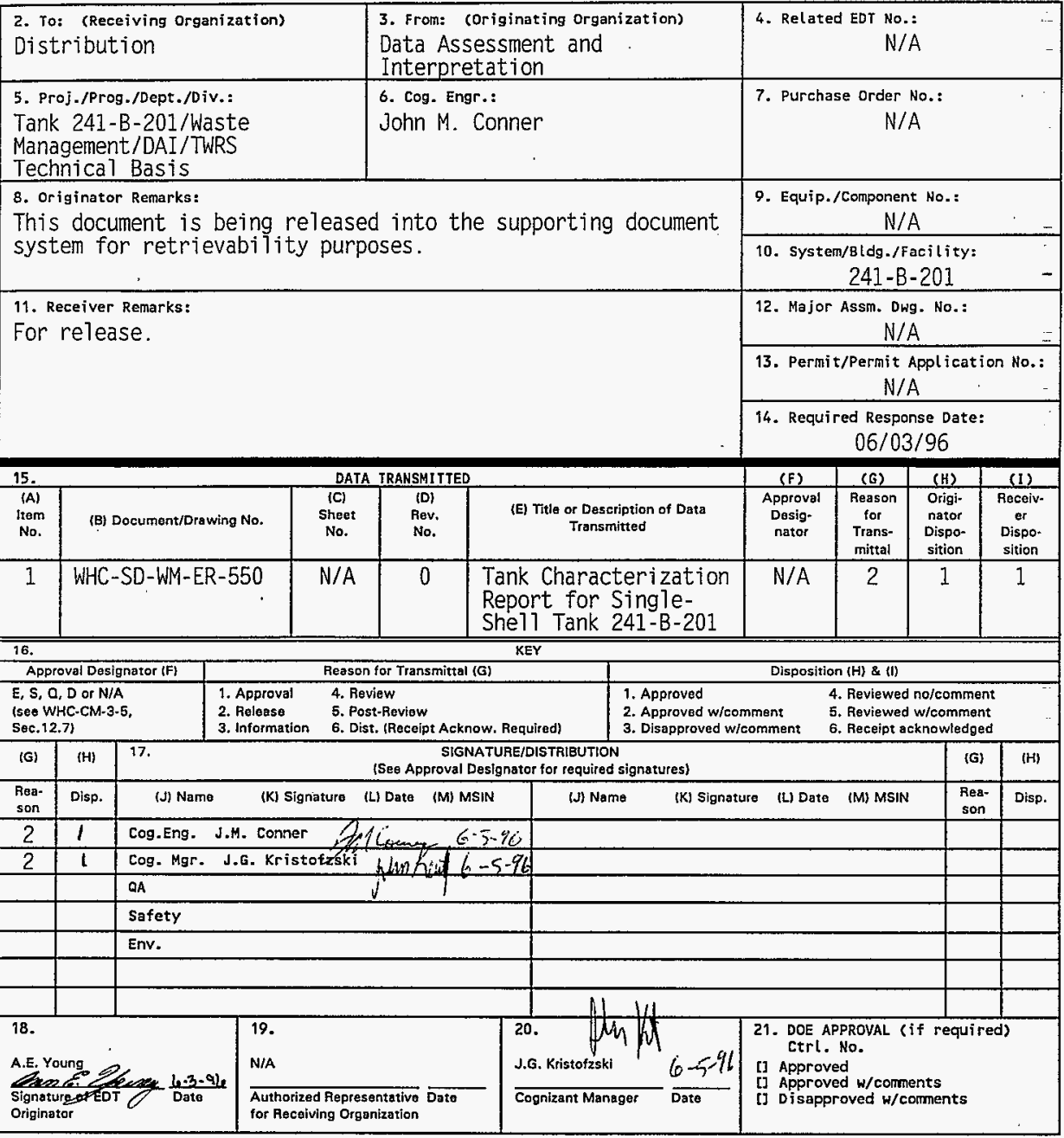

BD-7400-172-2 (04/94) GEF097 


\section{Tank Characterization Report for Single-Shell Tank 241-B-201}

John M. Conner

Westinghouse Hanford Company, Richland, WA 99352

U.S. Department of Energy Contract DE-AC06-87RL10930

EDT/ECN: EDT-615398

Org Code: 79400

UC: 2070

B\&R Code: EW 3120074 Total Pages: //8

Key Words: Tank 241-B-201, Tank B-201, B-201, B Farm, Tank Characterization Report. TCR. Single-Shel1

Abstract: This tank characterization report for Tank 241-B-201 was initially released as PNL-10100. This document is now being released as WHC-SD-WM-ER-550 in order to accommodate internet publishing.

TRADEMARK DISCLAIMER. Reference herein to any specific commercial product, process, or service by trade name, trademark, manufacturer, or otherwise, does not necessarily constitute or imply its endorsement, recommendation, or favoring by the United States Government or any agency thereof or its contractors or subcontractors.

Printed in the United States of America. To obtain copies of this document, contact: WHC/BCS Document Control Services, P.O. BoX 1970, Mailstop H6-08, Richland WA 99352, Phone (509) 372\%2420; Fax (509) 376-4989.
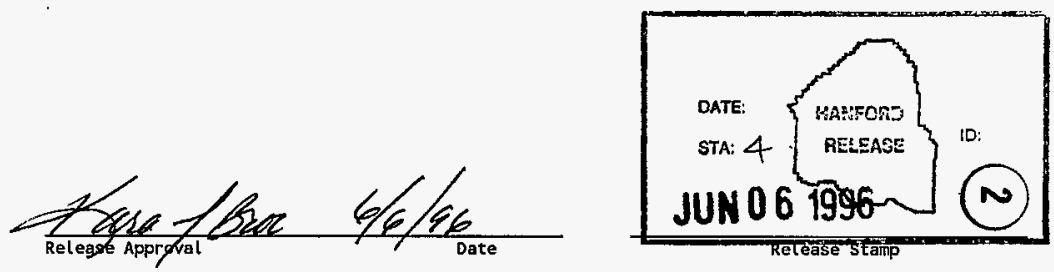

\section{Approved for Public Release}




\title{
Tank Characterization Report for Single-Shell Tank B-201
}

\author{
P. G. Heasler \\ K. M. Remund \\ J. M. Tingey \\ D. B. Baird \\ F. M. Ryan
}

Pacific Northwest National Laboratory

J. M. Conner

Westinghouse Hanford Company

Date Published

June 1996

Prepared for the U.S. Department of Energy

Assistant Secretary for Environmental Management

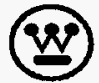

Westinghouse

P.O Box 1970

Hanford Company Richland, Washington

Management and Operations Contractor for the

U.S. Department of Energy under Contract DE-ACO6-87RL10930

Approved for Public Release; Distribution is Unlimited 


\section{Executive Summary}

Tank 241-B-201 (hereafter referred to as B-201) is a 208,000 liter $(55,000$ gallon) single-shell waste tank located in the 200 East B Tank Farm at Hanford. Two cores were taken from this tank in 1991 and analysis of the cores was conducted by Battelle's 325-A Laboratory in 1992. Characterization of the waste in this tank is being done to support the Hanford Federal Facility Agreement and Consent Order (Tri-Party Agreement), Milestone M-44-05 (Ecology 1993).

Tank B-201, located in the 200 East Area B Tank Farm, was constructed in 1943 and put into use in 1947. The history of this tank is relatively straightforward, compared to other tanks. For its entire lifetime, B-201 was used to hold wastes from the 224 facility, a lanthanum fluoride-based separation process that discharged wastes containing low concentration fission products. Waste from the 224 facility was pumped into this tank, allowed to settle, and the liquid effluent was allowed to overflow into cribs (long trenches engineered to receive waste material disposed into the soil). This tank was retired from service in 1975, and in 1980 surveillance data indicated the tank leaked 4,500 liters (1,189 gallons). The tank was consequently interim stabilized in 1981 . The tank presently consists of 110,000 liters $(29,000$ gallons) of sludge-like waste. Historically, there are no unreviewed safety questions associated with this tank and none were revealed by a review of the data from the latest core sampling event in 1991.

There were no difficulties encountered during the core sampling of this tank. Core recoveries were generally $100 \%$, and an extensive set of analytical measurements was run on 
the core composites. Core 26 was taken from riser 2 and core 27 was taken from riser 7 (see Figure A-1). Since core 26 was taken near a waste inlet/outlet (i.e., a place where the waste was frequently disturbed) and the core 27 was on the side of the tank far from the inlet, these two cores should represent the extremes of sampling conditions in the tank. In fact, the two cores exhibited many significant differences, and this core-to-core variability is the principal contributor to the uncertainty in the tank inventory estimates. For example, in the case of aluminum, the relative standard deviation (RSD) of the mean estimate is $74 \%$, caused in large part by a core-to-core RSD of $102 \%$.

The constituents found in highest concentration $(>10,000 \mu \mathrm{g} / \mathrm{g})$ in the B-201 core samples were water, bismuth, nitrate, sodium, silicon, manganese, lanthanum, iron, and calcium in descending order of concentration.

The results of the chemical analyses have been compared to the dangerous waste codes in the Washington Dangerous Waste Regulations (WAC 173-303). This assessment was conducted by comparing tank analyses against dangerous waste characteristics (" $D$ " waste codes) and against state waste codes. The comparison did not include checking tank analyses against "U," "P," "F," or "K" waste codes, since application of these codes is dependent on the source of the waste and not on particular constituent concentrations. The results indicate that the waste in this tank is adequately described in the Dangerous Waste Permit Application for the Single-Shell Tank System; this permit is discussed in De Lorenzo et al. (1994). 
Figure ES-1. Top View of Tank B-201.

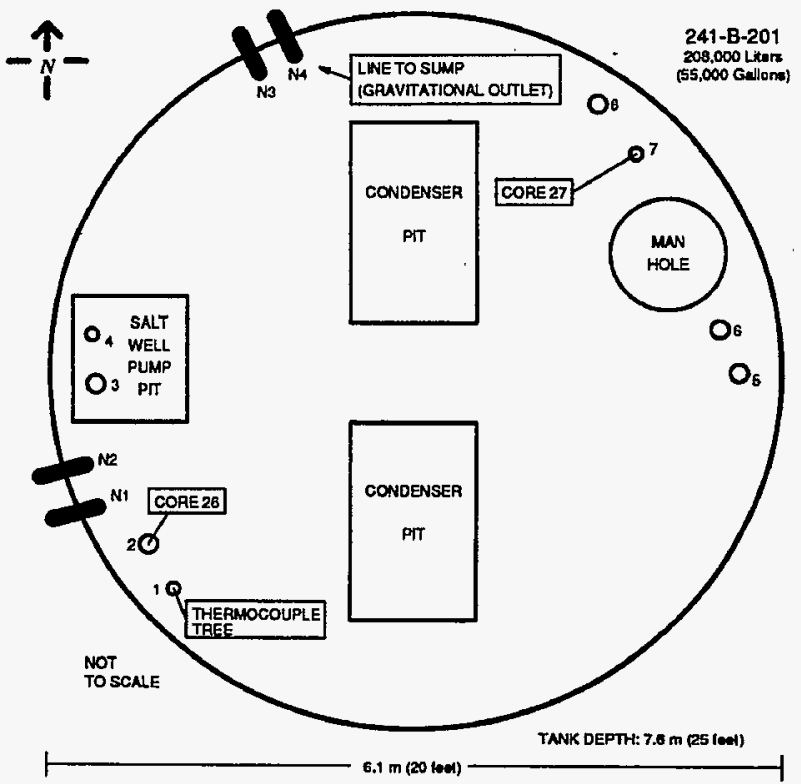

Table ES-1. Engineering Data Summary of Tank B-201.

\begin{tabular}{|c|c|c|c|}
\hline \multicolumn{2}{|c|}{ Tank Engineering Description } & \multicolumn{2}{|c|}{ Tank Status as of 1994} \\
\hline Type: & Single-shell tank & Watch List: & None \\
\hline Construction: & $1943-1944$ & Interim Stabilized: & 1981 \\
\hline In-Service: & 1947 & Interim Isolated: & 1981 \\
\hline Out of Service: & 1975 & Contents: & 224 waste* \\
\hline Diameter: & $6.1 \mathrm{~m}(20 \mathrm{ft})$ & Tank Integrity: & Assumed leaker $(1980)(4,500 \mathrm{~L})$ \\
\hline Depth: & $7.6 \mathrm{~m}(25 \mathrm{ft})$ & & \\
\hline Nominal Capacity: & $208,000 \mathrm{~L}(55,000 \mathrm{gal})$ & & \\
\hline Bottom Shape: & Dished & & \\
\hline Hanford Coordinates: & N45537.5, W52727.5 & & \\
\hline Ventilation: & Passive & & \\
\hline
\end{tabular}

*Non-complexed waste. 
Table ES-2. Inventory Summary for Tank B-201.

\begin{tabular}{|c|c|c|c|}
\hline \multicolumn{4}{|c|}{ Physical Properties of Waste } \\
\hline Total Waste: & $110,000 \mathrm{~L}(29000 \mathrm{gal})$ & Supernate Volume: & $3,800 \mathrm{~L}(1,000 \mathrm{gal})$ \\
\hline Drainable Inter. Liquid: & $11,400 \mathrm{~L}(3,000 \mathrm{gal})$ & Density: & $1.25 \mathrm{~g} / \mathrm{mL}$ \\
\hline $\mathrm{H}_{2} \mathrm{O}$ Average: & $60.6 \%$ & Temperature Average: & $17^{\circ} \mathrm{C}\left(63^{\circ} \mathrm{F}\right)$ \\
\hline pH: & 8.53 & Maximum Exotherm: & No Exotherms \\
\hline Heat Load: & $0.006 \mathrm{~kW}$ & & \\
\hline \multicolumn{4}{|c|}{ Chemical Properties of Waste } \\
\hline Bismuth: & $13,000 \mathrm{~kg}(9.45 \mathrm{wt} \%)$ & Calcium: & $1,670 \mathrm{~kg}(1.22 \mathrm{wt} \%)$ \\
\hline Nitrate: & $6,770 \mathrm{~kg}(4.93 \mathrm{wt} / \%)$ & Fluoride: & $800 \mathrm{~kg}(0.58 \mathrm{wt} \%)$ \\
\hline Sodium: & $5,240 \mathrm{~kg}(3.82 \mathrm{wt} \%)$ & Potassium: & $797 \mathrm{~kg}(0.58 \mathrm{wt} \%)$ \\
\hline Silicon: & $2,770 \mathrm{~kg}(2.02 \mathrm{wt} \%)$ & Phosphorus: & $748 \mathrm{~kg}(0.55 \mathrm{wt} \%)$ \\
\hline Manganese: & $2,630 \mathrm{~kg}(1.92 \mathrm{wt} \%)$ & TOC: & $71 \mathrm{~kg}(5.18 \mathrm{e}-02 \mathrm{wt} \%)$ \\
\hline Lanthanum: & $2,070 \mathrm{~kg}(1.51 \mathrm{wt} \%)$ & TIC: & $287 \mathrm{~kg}(0.21 \mathrm{wt} \%)$ \\
\hline Iron: & $1,840 \mathrm{~kg}(1.34 \mathrm{wt} \%)$ & & \\
\hline \multicolumn{4}{|c|}{ Radionuclides in the Waste } \\
\hline Total Plutonium: & $156 \mathrm{Ci}$ & Strontium-90: & $287 \mathrm{Ci}$ \\
\hline Cesium-137: & $110 \mathrm{Ci}$ & Total Uranium & $21.4 \mathrm{~kg}(1.56 \mathrm{e}-04 \mathrm{wt} \%)$ \\
\hline
\end{tabular}




\section{TABLE OF CONTENTS}

1.0 INTRODUCTION $\ldots \ldots \ldots \ldots \ldots \ldots \ldots \ldots \ldots \ldots \ldots \ldots \ldots \ldots \ldots \ldots$

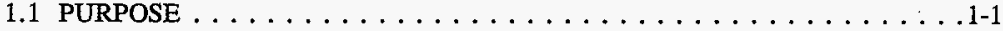

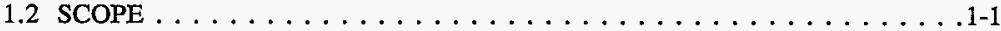

2.0 HISTORICAL TANK INFORMATION $\ldots \ldots \ldots \ldots \ldots \ldots \ldots \ldots \ldots \ldots \ldots$

2.1 TANK DESCRIPTION $\ldots \ldots \ldots \ldots \ldots \ldots \ldots \ldots \ldots \ldots \ldots \ldots \ldots \ldots .2-1$

2.2 PROCESS KNOWLEDGE $\ldots \ldots \ldots \ldots \ldots \ldots \ldots \ldots \ldots \ldots \ldots \ldots \ldots \ldots \ldots .2-1$

2.3 SURVEILLANCE DATA $\ldots \ldots \ldots \ldots \ldots \ldots \ldots \ldots \ldots \ldots \ldots \ldots \ldots \ldots .2-3$

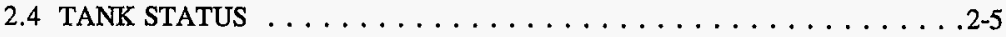

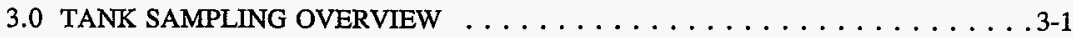

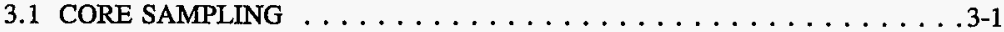

3.2 ADDITIONAL TANK SAMPLING $\ldots \ldots \ldots \ldots \ldots \ldots \ldots \ldots$

4.0 SAMPLE HANDLING AND ANALYTICAL SCHEME $\ldots \ldots \ldots \ldots \ldots \ldots$ 4-1

4.1 WASTE DESCRIPTION $\ldots \ldots \ldots \ldots \ldots \ldots \ldots \ldots \ldots . \ldots \ldots .1$

4.2 HOLDING TIME CONSIDERATIONS $\ldots \ldots \ldots \ldots \ldots \ldots \ldots .4 .4$

4.3 SAMPLE PREPARATION AND ANALYTICAL METHODS . . . . . . . 4-4

5.0 ANALYTICAL RESULTS AND WASTE INVENTORY . . . . . . . . . . 5-1

5.1 CHEMICAL ANALYSES AND RADIOLOGICAL DETERMINATIONS . . . 5-1

5.2 PHYSICAL MEASUREMENTS $\ldots \ldots \ldots \ldots \ldots \ldots \ldots \ldots \ldots$ 5-12

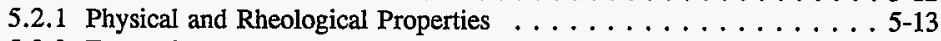

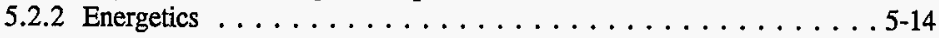

5.2 .3 Particle Size Analysis $\ldots \ldots \ldots \ldots \ldots \ldots \ldots \ldots \ldots \ldots$. . . . . . . . .

5.3 HEAT LOAD ANALYSIS $\ldots \ldots \ldots \ldots \ldots \ldots \ldots \ldots \ldots \ldots \ldots$

6.0 INTERPRETATION OF ANALYTICAL RESULTS . . . . . . . . . . 6-1

6.1 TANK WASTE PROFILE $\ldots \ldots \ldots \ldots \ldots \ldots \ldots \ldots \ldots \ldots \ldots .1$

6.2 WASTE SUMMARY AND CONDITIONS $\ldots \ldots \ldots \ldots \ldots \ldots \ldots$

7.0 STATISTTCAL QUALITY ASSURANCE TESTS $\ldots \ldots \ldots \ldots \ldots \ldots \ldots$ 7-1

7.1 MASS AND CHARGE BALANCE $\ldots \ldots \ldots \ldots \ldots \ldots \ldots \ldots \ldots$.1

7.2 HOMOGENIZATION TESTS $\ldots \ldots \ldots \ldots \ldots \ldots \ldots \ldots .7 .4$

7.3 EVALUATION OF SPIKES AND BLANKS $\ldots \ldots \ldots \ldots \ldots \ldots 7-6$

7.3.1 Blank Measurements . . . . . . . . . . . . . . .7-7

7.3.2 Spike Measurements . . . . . . . . . . . . . . 7-8

7.3.3 Quality Assurance Flags $\ldots \ldots \ldots \ldots \ldots \ldots \ldots \ldots$. . . . . . .

8.0 CONCLUSIONS AND RECOMMENDATIONS $\ldots \ldots \ldots \ldots \ldots \ldots$. 1 


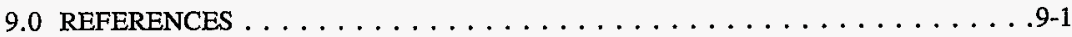

10.0 BIBLIOGRAPHY $\ldots \ldots \ldots \ldots \ldots \ldots \ldots \ldots \ldots \ldots \ldots \ldots \ldots \ldots \ldots \ldots$

\section{APPENDIXES}

A TANK ENGINEERING DATA AND WASTE SUMMARY $\ldots \ldots \ldots \ldots$ A-1

B COMPOSITE ESTIMATES AND VARIABILITY SUMMARY . . . . . . B-1

C SEGMENT-LEVEL ESTIMATES AND VARIABILITY SUMMARY $\ldots \ldots$ C-1

D RAW DATA SET SUMMARY $\ldots \ldots \ldots \ldots \ldots \ldots \ldots \ldots \ldots \ldots$ D- 1

E RHEOLOGICAL PROPERTIES TABLES AND PLOTS $\ldots \ldots \ldots \ldots$ E-1

\section{LIST OF FIGURES}

2-1 Tank Waste Level Summary for B-201 . . . . . . . . . . . . . . 2-3

2-2 Thermocouple Data for B-201 . . . . . . . . . . . . . 2-4

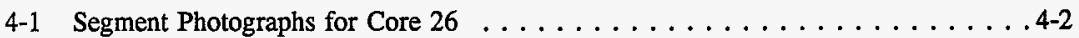

4-2 Segment Photographs for Core $27 \ldots \ldots \ldots \ldots \ldots \ldots \ldots \ldots .4 \ldots \ldots \ldots$

4-3 Sample Preparation Flowchart $\ldots \ldots \ldots \ldots \ldots \ldots \ldots \ldots . . \ldots \ldots .4$

5-1 RSD Distributions for Variance Components Calculated from the Composite-Level Data $\ldots \ldots \ldots \ldots \ldots \ldots \ldots \ldots \ldots \ldots$. . . . . . . . . .

5-2 Residuals Plot for ICP Acid Digestion Aluminum Analysis $\ldots \ldots \ldots \ldots$ 5-11

6-1 RSD Distributions for Variance Components Calculated from the Segment-Level Data . . . . . . . . . . . . . . . . 6-7

7-1 Boxplots of Blank/Actual for Each Analytical Method ( $<D L$ Measurements Excluded) . . . . . . . . . . . . . . . . 7-8

7-2 Boxplots \% Recovery Calculated from Spike Measurements . . . . . . . . 7-9 


\section{LIST OF TABLES}

2-1 Estimated Composition of B-201 Contents $\ldots \ldots \ldots \ldots \ldots \ldots \ldots \ldots \ldots \ldots$

3-1 Actual Percent Recovery in B-201 . . . . . . . . . . . . . . 3-2

3-2 Historical Core Sample Analytical Data for B-201 . . . . . . . . . . 3-3

4-1 Sample Preparation and Analytical Methods Used on B-201 Samples . . . . . . 4-6

5-1 Summary of B-201 Analytical Result Counts . . . . . . . . . . . 5-1

5-2 Summary of the Composite Level Results for Anions, Metals, Organics, and Radionuclides . . . . . . . . . . . . . . . . . . . . 5-2

5-3 Analytes Grouped According to Concentration Differences Between Cores . . . . 5 5-9

5-4 Composite Values Omitted from Analyses as Suspect . . . . . . . . . . . 5-10

5-5 Summary of Core 26 Physical Measurements . . . . . . . . . 5-12

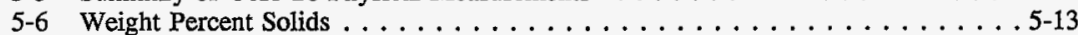

5-7 Core 27 Differential Scanning Calorimetry Thermal Measurements . . . . . 5-15

5-8 Particle Size Distribution for Core $27 \ldots \ldots \ldots \ldots \ldots \ldots \ldots \ldots$. . . . . . . . . . .

5-9 Radionuclide Inventory and Projected Heat Load . . . . . . . . . . . 5-16

6-1 Available Segment-Level Measurements . . . . . . . . . . . . . . 6-2

6-2 Summary of RSD Values . . . . . . . . . . . . . . . . 6-3

6-3 Analytes Classified by Dominant RSD . . . . . . . . . . . . . . 6-4

6-4 Comparison of Mean Tank Estimates (Historical versus Sampling Results) . . . . . 6-5

6-5 Alpha and Beta Energy Checks $\ldots \ldots \ldots \ldots \ldots \ldots \ldots \ldots \ldots .6 \ldots \ldots .6 \ldots$

7-1 Mass/Charge Balance for Metals . . . . . . . . . . . . . . 7-1

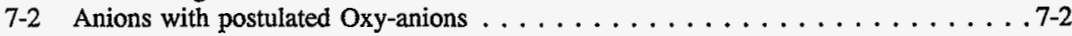

7-3 Phosphate Solubility . . . . . . . . . . . . . . . . . .

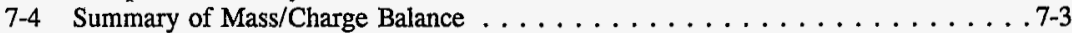

7-5 Homogenization Test Results $\ldots \ldots \ldots \ldots \ldots \ldots \ldots \ldots \ldots . . \ldots \ldots$

7-6 Illustration of Contamination Problem for ICP fusion on Core $26 \ldots \ldots \ldots \ldots 7-6$

7-7 Summary of Blank Measurements . . . . . . . . . . . . . 7-7

7-8 Summary of Recoveries Calculated from Spike Measurements $\ldots \ldots \ldots \ldots$ 7-10 


\section{LIST OF TERMS}

AA

ANOVA

CVAA

GC

GEA

HAS

ICP

LANL

PNNL

QA

RPD

RSD

SAL

SST

TCLP

TIC

TOC

TRAC

Tri-Party Agreement

WHC atomic absorption

analysis of variance

cold vapor atomic absorption

gas chromatography

gamma energy analysis

Hanford Analytical Services

inductively coupled plasma atomic emmission spectrometry

Los Alamos National Laboratory

Pacific Northwest National Laboratory

quality asssurance

relative percent difference

relative standard deviation

Shielded Analytical Laboratory

single-shell tank

toxicity characteristic leach procedure

total inorganic carbon

total organic carbon

Track Radioactive Components (model)

Hanford Federal Facility Agreement and Consent Order

Westinghouse Hanford Company 


\section{Acknowledgements}

It is appropriate to acknowledge individuals that made important contributions to this report. Brett Simpson (Westinghouse Hanford Company) provided many valuable comments and recommendations that helped prepare this report. Stacey Hartley spent many hours preparing this report for its final edition. Daryl Baird wrote several computer programs that helped in statistical analysis of the B-201 data. We also appreciate the help of Chris Brevick (ICF Kaiser Hanford Company) and Todd Brown (Westinghouse Hanford Company) for providing much of the historical information that was needed for this report. 
This page intentionally left blank. 


\subsection{INTRODUCTION}

Analysis was conducted on materials obtained from tank B-201 to complete the Hanford Federal Facility Agreement and Consent Order (Tri-Party Agreement), Milestone M-44-05 (Ecology 1993) to sample, analyze, and report on 20 tanks. Other objectives that these measurements and inventory estimates support are as follows:

- Obtain estimates of both the concentration and total quantity of key analytes relating to safety issues such as organics and radionuclides.

- Provide input to risk-assessment-based decisions for disposal of the waste.

- Implement physical property measurements such as rheology, bulk density, and particle size.

These measurements are necessary for the design and fabrication of retrieval, pretreatment, and final waste disposal systems.

\subsection{PURPOSE}

The purpose of this report is to characterize the waste in single-shell tank (SST) B-201. "Characterization" includes the determination of the physical, chemical (e.g., concentrations of elements and organic species), and radiological properties of the waste. These determinations are made using analytical results from B-201 core samples as well as historical information about the tank. The main objective is to determine average waste properties; but in some cases, concentrations of analytes as a function of depth were also determined.

This report also consolidates the available historical information regarding tank B-201, arranges the analytical information from the recent core sampling in a useful format, and provides an interpretation of the data within the context of what is known about the tank.

\subsection{SCOPE}

The waste properties are determined from core samples, which were chemically analyzed by the Pacific Northwest National Laboratory (PNNL, 325-A Laboratory). Additional relevant information on the waste has been compiled from historical sources. Types of historical information that are routinely checked include:

- Past sampling events

- Routine tank surveillance measurements

- Tank transfer records. 
This historical information has been reviewed and compared with the laboratory data to help interpret the laboratory data correctly. However, the characterization estimates presented are derived from the laboratory data unless otherwise indicated. The laboratory data is considered to provide the most authoritative description of the tank waste.

The minimum required sampling and analysis was performed on tank B-201. This sampling is intended to determine mean concentrations (through composite analysis) and is thus intended to meet process design characterization objectives for waste treatment. Process design generally requires knowledge of total inventories. However, a few measurements were made to predict the spatial distribution of specific analytes in the tank. 


\subsection{HISTORICAL TANK INFORMATION}

Since 1944, underground storage tanks in Hanford's 200 Areas have been used to store radioactive waste generated by processing plants and laboratories on the Hanford Site. A study of waste management operation records yields information about the process waste types transferred into a tank and the physical state of the waste. By reviewing the plant effluent stream composition, transfer records, and the subsequent history of the service life of a tank, hypotheses about the expected contents of the tank can be made.

The B Tank Farm is located in the 200 East Area and was constructed during 1943 and 1944. The B Tank Farm is one of the original four tank farms (B, C, T, and U) made up of SSTs. There are 16 waste tanks in B Farm. Four tanks (B-201 to B-204) have a nominal capacity of 208,000 liters (55,000 gallons). Tanks B-202, B-203, and B-204 are connected together via tie lines whereas tank B-201 is not. The remaining 12 tanks (B-101 to B-112) have a capacity of $2,006,000$ liters $(530,000$ gallons).

\subsection{TANK DESCRIPTION}

A summary of the basic design for tank B-201 is presented in Appendix A. As can be seen from the schematics presented in the appendix, B-201 is a small tank with a capacity of 208,000 liters. The tank is buried under approximately 2 meters ( 6.5 feet) of earth for shielding purposes and is not connected in cascade or by tie lines with any other tanks. The tank was classified as a leaker in 1980 and it is estimated to have leaked approximately 4,500 liters ( 1,189 gallons) of waste.

\subsection{PROCESS KNOWLEDGE}

The process history for this tank is relatively straightforward. Tank B-201, as well as the other three 208,000-liter tanks in B Farm (B-202 through B-204), received waste from essentially one source, the $L_{a F_{3}}$ Plutonium Concentration Process (one of the final steps in the Bismuth Phosphate Process) located in the 224 Building. This "224-waste" has a very low radionuclide content (low enough to permit ground disposal). When tank B-201 was in operation, the waste from the 224 Building was run into the tank and the solids were allowed to settle out. The liquid waste was then directed into a crib, a long trench engineered to receive wastes for disposal into the soil. An estimated 110,000 liters (29,000 gallons) of this waste is currently in the tank and its historical composition is as described in Table 2-1. These estimates were derived by Los Alamos National Laboratory (LANL) and are tabulated in Brevick 1994. The numbers given in Table 2-1 are the estimated composition of the waste in B-201, rather than the composition of the waste stream from the 224 Building. 
Table 2-1. Estimated Composition of B-201 Contents.

\begin{tabular}{|c|c|c|c|c|c|}
\hline Analyte & $\overline{\mathbf{M}}$ & $\mu \mathrm{g} / \mathrm{g}$ & Analyte & $\mathbf{M}$ & $\mu \mathrm{g} / \mathrm{g}$ \\
\hline Sodium & 1.906 & 39018 & Free Hydroxide & 0.015 & 230 \\
\hline Nitrate & 1.292 & 71304 & Carbonate & 0.000 & 0 \\
\hline Hydroxide & 0.556 & 8419 & Sulfate & 0.000 & 0 \\
\hline Fluoride & 0.303 & 5132 & Calcium & 0.000 & 0 \\
\hline Potassium & 0.259 & 9014 & Chloride & 0.000 & 0 \\
\hline Oxalate & 0.214 & 16800 & Nitrite & 0.000 & 0 \\
\hline Phosphate & 0.201 & 17001 & Acetate & 0.000 & 0 \\
\hline Bismuth & 0.159 & 29581 & Uranium & 0.000 & 0 \\
\hline Manganese & 0.117 & 5770 & TOC & 0.458 wt\% & 4580 \\
\hline Chromium & 0.023 & 1068 & & & \\
\hline Plutonium & & $0.964 \mu \mathrm{Ci} / \mathrm{g}$ & Density & $1.123 \mathrm{~g} / \mathrm{mL}$ & \\
\hline Cesium & & $0.000 \mu \mathrm{Ci} / \mathrm{L}$ & Water & $80.140 \%$ & \\
\hline Strontium & & $0.000 \mu \mathrm{Ci} / \mathrm{L}$ & & & \\
\hline
\end{tabular}

Based on LANL's historical tank composition estimates, it is expected that the waste in B-201 should be very similar to the waste in B-202, B-203, and B-204.

The Hanford Works Monthly Reports for February and March of 1948 (HW-9191 and $\mathrm{HW}-9595)$ indicate that $\mathrm{B}-201$ received cell drainage waste from cell 5 , tank 6 in B Plant (i.e., 5-6 waste). When any of the subprocesses of the Bismuth Phosphate Process boiled over, the waste drained to cell 5 , tank 6 . Since the cell could have received waste from several of these subprocesses, little is known about the composition of this waste type. The amount of 5-6 waste that was received by B-201 is also unknown. No attempt was made to determine the impact that the 5-6 waste had on the tank contents.

B-201 started receiving waste from the 224 Building in 1947. By the end of the first quarter of 1952 , B-201 had received at least 206,000 liters $(54,500$ gallons) of 224-waste, and was actively cascading into a crib. In the second quarter of 1958 , the solid portion of the waste was estimated to be 106,000 liters $(28,000$ gallons). In the second quarter of $1971,83,000$ liters (22,000 gallons) of supernate were transferred out of tank B-201 to tank B-106. During 1974 and 1975, five smaller supernate transfers (4,000 to 23,000 liters [1,057 to 6,076 gallons]) were made from B-201 to B-109. In the fourth quarter of 1974 , 15,000 liters $(3,963$ gallons) of water were added to the tank and in the third quarter of 1975, B-201 was removed from service.

The total tank waste volume remained basically constant at 110,000 liters from the third quarter of 1975 to the present time, with the exception of the assumed leak of 
4,500 liters in 1980. The total volume of solids in the tank is 106,000 liters and the total volume of supernate liquid is approximately 3,800 liters (1,004 gallons). Jungfleisch and Simpson 1993, also obtained tank content estimates for B-201 (via the Track Radioactive Components model [TRAC]). However, all of the estimates supplied by TRAC were zero, and therefore, TRAC results are not included here for comparison to the LANL historical estimates. (See Figure 2-1).

Figure 2-1. Tank Waste Level Summary for B-201.

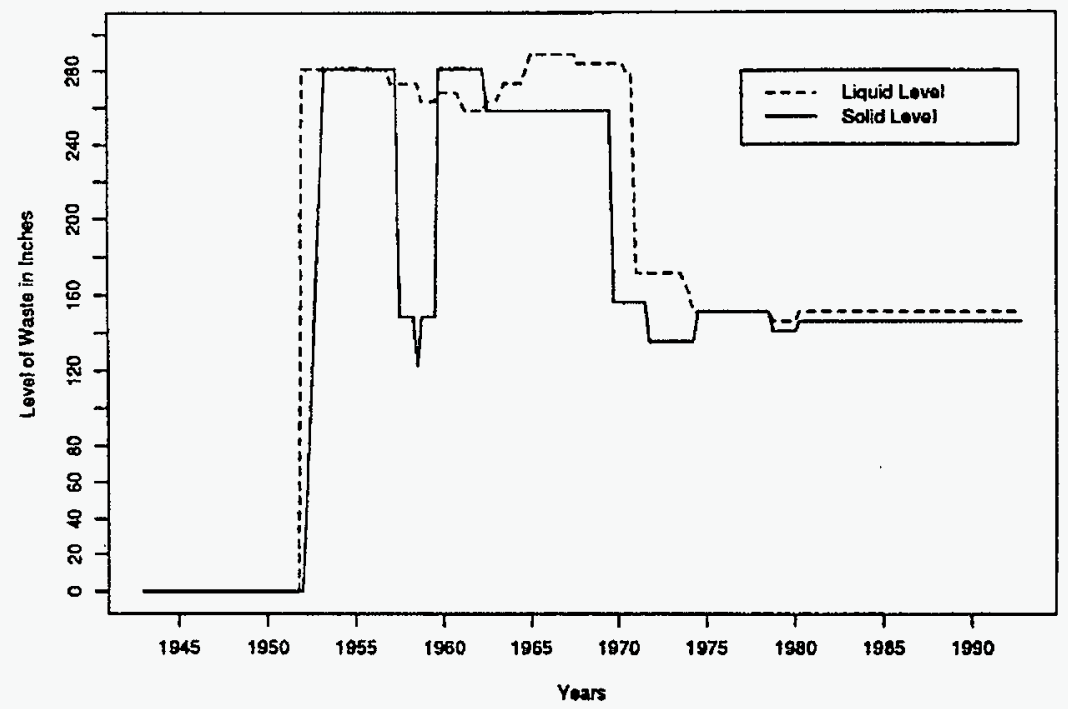

\subsection{SURVEILLANCE DATA}

Each of the 177 underground tanks at the Hanford Site is routinely monitored for changes in supernate levels, solid waste levels, and temperature readings. A monthly surveillance report lists the results of this monitoring and the status of each tank (e.g., watch lists, leak status, unusual events).

A possible discrepancy can be seen in the data between 1952 and 1972 . The solids levels reported over this time period rise as high as 7.3 meters $(204,000$ liters). It is doubtful that the solids level ever rose above 3.8 meters (approximately 110,000 liters). This is due to the fact that approximately 117,000 liters $(30,908$ gallons) of supernate were 
transferred from B-201 to B-106 and B-109 between 1971 and 1974 (Anderson 1990). After these transfers, the reported levels for supernate and solids dropped to the same level now observed (110,000 liters). Given that supernate was all that was removed from the tank, it is reasonable to assume that the solids level never rose above 3.8 meters. Another point that adds credence to the last statement is that the reported solids levels during 1958 and 1959 dropped to approximately 110,000 liters, even though no tank transfer activity is reported during that same time period.

Figure 2-2 is a plot of the B-201 temperature data recorded from 1975 to the present. With the exception of a group of readings in July 1989, the temperatures have remained relatively stable over time. Note that there are no recorded temperature readings from 1980 through 1988. In the last group of data from 1990 to the present, temperatures deviate from $17^{\circ} \mathrm{C}\left(63^{\circ} \mathrm{F}\right)$ by $\pm 5.5^{\circ} \mathrm{C}\left( \pm 10^{\circ} \mathrm{F}\right)$. Within a given year during this time period, temperatures deviate from the average reading by $\pm 3^{\circ} \mathrm{C}\left( \pm 6^{\circ} \mathrm{F}\right)$. The range of temperatures measured in B-201 is not high enough to warrant any concern about high heat evolution.

Figure 2-2. Thermocouple Data for B-201.

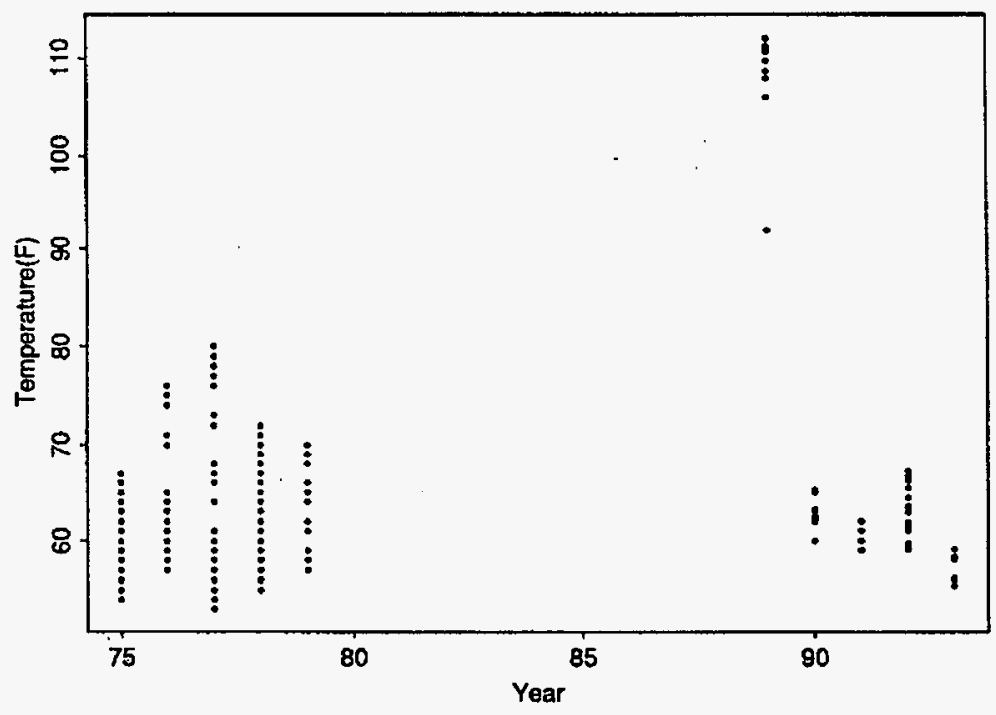




\subsection{TANK STATUS}

B-201 is presently not on any watch list and has no unreviewed safety issues. B-201 is an interim isolated tank, which means that all access to the tank not required for long-term surveillance has been sealed in a way that provides at least one barrier to the inadvertent addition of liquid. This tank is also interim stabilized. This means that B-201 is an inactive waste storage tank, and that as much of the free liquid as possible has been removed with a salt well pump. B-201 was put on the assumed leaker list after an observed drop in the waste surface level (approximately 4,500 liters) was detected in 1980. 
WHC-SD-WM-ER-550 Rev 0

This page intentionally left blank. 


\subsection{TANK SAMPLING OVERVIEW}

This section describes the latest tank B-201 core sampling event (1991) and another core sampling event in 1978. For the 1991 sampling of tank B-201, details are given about the core sampling equipment, the sampling process, the location of samples taken from the tank, the sample recoveries, and the transfer of the core samples to the 325-A Laboratory.

\subsection{CORE SAMPLING}

All Hanford waste tanks are underground tanks, usually buried under 2 meters ( 6.5 feet) of earth. Consequently, access to the waste is limited to existing risers, as illustrated in Appendix A. Special core sampling equipment, which is mounted on trucks, is used to take cores from the tank. During sampling, the truck is positioned over the desired riser and a "drill-string" containing the sampler is lowered into the tank. Two types of samplers are used at Hanford, push mode and rotary mode. The rotary mode samplers are much more effective in producing a complete sample of the waste (a rotary mode sampler can actually be used in push or rotary mode). However, the rotary mode sampler takes more time to set up, and there are some safety concerns associated with it (e.g., generation of heat at the drill bit and potential ignition of the waste). Therefore, push mode sampling is used whenever possible. Since the waste was expected to be relatively soft, push mode sampling was used in tank B-201.

Several factors enter into the decision of which risers to sample. When possible, samples are taken from risers that are widely spaced, usually on opposite sides of the tank. This choice provides some information on the lateral variation of the waste.

The sampler is constructed of stainless steel and is $48 \mathrm{~cm}$ (19 in.) long, with a $2.2 \mathrm{~cm}$ (7/8 in.) inside diameter, and has a volume of $187 \mathrm{~mL}$. The sampler is fitted with a piston inside the cylindrical sample reservoir to control entry of the waste. As the drill string is lowered, the piston recedes from the sample reservoir and creates a partial vacuum that pulls the sample into the waste column. The sampler fits into a 5-cm (2-in.) drill pipe fitted with a blunt drill bit. After each segment is filled, the sampler is extracted from the drill pipe and another sampler is inserted. The drill string is then lowered another $48 \mathrm{~cm}$ (19 in.).

Some deficiencies associated with the sampler can have an impact on the representativeness of the sample. For example, because of the location of the risers, the sampler cannot extract the waste in the dished portion of the tank. Since the waste at the bottom of the tank may be significantly different from the rest, this can cause an important bias. Also, the sampler sometimes gets plugged and may gather much less than the standard $48 \mathrm{~cm}$ sample. Percent recovery estimates on a core or segment basis, express how completely the material has been sampled. 
After a segment is captured by the sampler, it is sealed within a stainless steel liner and placed in a shipping cask. The casks are transported to the analytical laboratory for analysis and storage.

Two core samples were taken from tank B-201 at opposite ends of the tank in July, 1991 (core 26) and August, 1991 (core 27). Core 26 was taken through riser 2, which is near the waste inlet pipe. Core 27 was taken through riser 7 . The strategy behind this selection of risers is simple; since the tank history indicates that the tank was used to settle solids from 224-waste and that this waste has not been disturbed by sluicing or by any tank transfers, the coarser solids should have settled out at core 26 and the finer at core 27 . The two cores should therefore provide us with estimates of the extremes occurring in the waste. Ideally, the waste make-up between the two cores should vary in a linear fashion. If these assumptions concerning the waste are correct, these core locations should be optimal for producing an estimate of average waste make-up.

The waste in the tank is relatively deep ( 4 meters [13 feet]). In both cores, 8 segments were required to produce a complete column of the waste. Segment 1 is taken from the top of the waste, and segment 8 is a bottom sample.

In this tank, there were no recovery problems, and the sampler recovered essentially $100 \%$ of the core and segment samples. Segment 1 of core 26 has the lowest recovery, $65 \%$. While this may seem low, the typical sampling protocol is to only partially fill the first segment of a sample, so that the rest of the waste column can be divided into whole segments. In fact, the planned recovery for this segment was $70 \%$. The planned recovery for all other segments was $100 \%$. Table $3-1$ presents the percent recoveries achieved, along with the planned recovery for the first segment.

Table 3-1. Actual Percent Recovery in B-201.

\begin{tabular}{|c|rr|}
\hline Segment & Core 26 & \multicolumn{1}{c|}{ Core 27 } \\
\hline 1 & $65 \% / 70 \% *$ & $100 \% / 100 \% *$ \\
\hline 2 & $95 \%$ & $100 \%$ \\
\hline 3 & $100 \%$ & $100 \%$ \\
\hline 4 & $100 \%$ & $100 \%$ \\
\hline 5 & $100 \%$ & $100 \%$ \\
\hline 6 & $100 \%$ & $100 \%$ \\
\hline 7 & $100 \%$ & $100 \%$ \\
\hline 8 & $100 \%$ & $100 \%$ \\
\hline
\end{tabular}

*Actual/Planned. 
The sample casks were transported to the 325-A Laboratory for extrusion and characterization analysis. The 325-A Laboratory is operated by Battelle-PNNL in the 300 Area of the Hanford Site. Core 26 segments 1,2 , and 3 were delivered to the 325-A Laboratory on July 25, 1991; core 26 segments 7 and 8 were delivered on July 29, 1991; core 26 segments 4,5 , and 6 were delivered on July 30, 1991; core 27 segments 1, 2, and 3 were delivered on August 5, 1991; core 27 segments 4, 5, and 6 were delivered on August 7, 1991; and core 27 segments 7 and 8 were delivered on August 8, 1991.

\subsection{ADDITIONAL TANK SAMPLING}

In 1978, core samples were taken from all four of the 55,000-gallon tanks in B Tank Farm (B-201, B-202, B-203, and B-204). There is no information indicating where the core samples were taken within the tanks. These core samples were sent to the Chemical Sciences Group for characterization (Horton 1978).

The chemists noted that the core samples were black in color and had the consistency of soft grease. There is no information about core recovery percentages. However, personnel who operated core sampling equipment during that time period indicated that the equipment operated reasonably well in the type of waste described by the chemists. Table 3-2 contains the analytical results from the core sample taken from B-201 in 1978. The analytical results reported for the other three tanks are similar to the B-201 results. A comparison of the results from these samples to the historical estimates and the analytical results from the 1991 core sampling event will follow in Section 5.1.

Table 3-2. Historical Core Sample Analytical Data for B-201. (2 pages)

\begin{tabular}{|lrr|}
\hline \multicolumn{1}{|c}{ Analyte } & Water Soluble & Acid (Fusion) \\
\hline $\mathrm{Al}$ & $0.05 \%$ & $1.0 \%$ \\
\hline $\mathrm{Bi}^{3+}$ & $<0.005 \%$ & $3.8 \%$ \\
\hline $\mathrm{CO}_{3}^{2-}$ & $3.3 \%$ & $\mathrm{NR}$ \\
\hline $\mathrm{CrO}_{4}^{-}$ & $0.05 \%$ & $\mathrm{NR}$ \\
\hline $\mathrm{Cl}^{-}$ & $0.01 \%$ & $\mathrm{NR}$ \\
\hline $\mathrm{Fe}$ & $<0.0002 \%$ & $1.8 \%$ \\
\hline $\mathrm{Hg}$ & $0.05 \%$ & $\mathrm{NR}$ \\
\hline $\mathrm{K}$ & $0.3 \%$ & $\mathrm{NR}$ \\
\hline $\mathrm{La}^{3+}$ & $<0.003 \%$ & $1.3 \%$ \\
\hline $\mathrm{Mn}^{3+}$ & $<0.003 \%$ & $\mathrm{NR}$ \\
\hline $\mathrm{Ni}^{2+}$ & & $0.1 \%$ \\
\hline $\mathrm{NO}_{2}^{-}$ & $0.004 \%$ & $\mathrm{NR}$ \\
\hline
\end{tabular}


WHC-SD-WM-ER-550 Rev 0

Table 3-2. Historical Core Sample Analytical Data for B-201. (2 pages)

\begin{tabular}{|lrr|}
\hline \multicolumn{1}{|c}{ Analyte } & Water Soluble & \multicolumn{1}{c|}{ Acid (Fusion) } \\
\hline $\mathrm{NO}_{3}^{-}$ & $3.3 \%$ & $\mathrm{NR}$ \\
\hline $\mathrm{Na}^{+}$ & $2.8 \%$ & $\mathrm{NR}$ \\
\hline $\mathrm{OH}^{-}$ & $0.6 \%$ & $\mathrm{NR}$ \\
\hline $\mathrm{PO}_{4}^{3-}$ & $0.05 \%$ & $1.1 \%$ \\
\hline $\mathrm{SO}_{4}^{2-}$ & $<0.01 \%$ & $<0.06 \%$ \\
\hline $\mathrm{SiO}_{2}^{2-}$ & $0.05 \%$ & $0.3 \%$ \\
\hline $\mathrm{U}$ & $7.41 \mathrm{E}-06 \mathrm{~g} / \mathrm{g}$ & $1.05 \mathrm{E}-05 \mathrm{~g} / \mathrm{g}$ \\
\hline $\mathrm{Pu}$ & $<1.29 \mathrm{E}-10 \mathrm{~g} / \mathrm{g}$ & $5.00 \mathrm{E}-05 \mathrm{~g} / \mathrm{g}$ \\
\hline $\mathrm{Am}$ & $5.69 \mathrm{E}-12 \mathrm{~g} / \mathrm{g}$ & $1.33 \mathrm{E}-09 \mathrm{~g} / \mathrm{g}$ \\
\hline${ }^{89+90} \mathrm{Sr}^{2+}$ & $2.10 \mathrm{E}-03 \mu \mathrm{Ci} / \mathrm{g}$ & $2.70 \mu \mathrm{Ci} / \mathrm{g}$ \\
\hline${ }^{137} \mathrm{Cs}^{+}$ & $0.05 \mu \mathrm{Ci} / \mathrm{g}$ & $0.059 \mu \mathrm{Ci} / \mathrm{g}$ \\
\hline${ }^{155} \mathrm{Eu}^{2+}$ & $\mathrm{NR}$ & $0.028 \mu \mathrm{Ci} / \mathrm{g}$ \\
\hline $\mathrm{Ce}$ & $\mathrm{NR}$ & $0.016 \mu \mathrm{Ci} / \mathrm{g}$ \\
\hline \hline Water Solubility & $\mathrm{NR}$ & $23.0 \%$ \\
\hline Bulk Density & $\mathrm{NR}$ & $1.37 \mathrm{~g} / \mathrm{cc}$ \\
\hline Percent Water & & $72.2 \%$ \\
\hline
\end{tabular}

NR: Not Reported 


\subsection{SAMPLE HANDLING AND ANALYTICAL SCHEME}

The sample handling and sample breakdown process and the analyses performed on each portion of the waste are described in this section. These analyses were performed on two core samples from tank B-201, core 26 and core 27. Each core sample consisted of eight 48-cm (19-in.) segments. The segments are numbered from 1 at the top of the core sample down to number 8 at the bottom.

\subsection{WASTE DESCRIPTION}

Eight segments of material from core 26 were received and extruded at PNNL's HighLevel Radiochemistry Facility (325-A Hot-Cell Facility). Recoveries of $100 \%$ were achieved for all segments except segment 1 (65\% recovery) and segment 2 (95\% recovery). Segment 1 contained $57 \mathrm{~mL}$ of drainable liquid. The mass of the drainable liquid was $55.7 \mathrm{~g}$ and the density was $1.0 \mathrm{~g} / \mathrm{mL}$. No other segments from core 26 contained drainable liquids. The first portion of the drainable liquid to be extruded was tan to gray in color. This liquid was followed by a dark brown liquid. All of the drainable liquid was opaque. The solids obtained from segments 1 and 2 were sticky dark brown sludges which held their shape upon extrusion from the sampler. The consistency of the solids from these two segments varied from soft at the top to crumbly at the bottom of segment 2 . The remainder of the sludge (segments 3 through 8 ) had a smooth texture, but extruded in chunks. The color of the core material gradually changed from dark brown to black as a function of the sample's depth in the tank. Segments 3 and 4 were dark brown to charcoal in color, while segments 5 through 8 were charcoal to black. The core material contained a significant amount of moisture, and in some spots in segment 7 it appeared that there were small pockets (less than $1 \mathrm{~mL}$ ) of liquid trapped in the sludge.

Eight segments of material from core 27 were received and extruded. Recoveries of $100 \%$ were achieved for each of the segments. The majority of the core was a black moist sludge which held its shape upon extrusion. The core material contained a significant amount of moisture, but no drainable liquids were obtained. The top 5 to $7.6 \mathrm{~cm} \mathrm{(2} \mathrm{to}$ $2.5 \mathrm{in.)}$ of the core were dark brown, and the remainder of the core was shiny black. The core material was fairly stiff except for the top $2.5 \mathrm{~cm}$ (1 in.) of segments $2,3,4$, and 5 , which flowed upon extrusion.

Each segment from both cores was photographed in the extrusion tray. Figure 4-1 shows the segments for core 26 and Figure 4-2 shows the segments for core 27 . For core 26, segments 1 through 8 are labeled 91-042 through 91-049, respectively. For core 27 , segments 1 through 8 are labeled $90-050$ through $90-057$, respectively. 
Figure 4-1. Segment Photographs for Core 26.
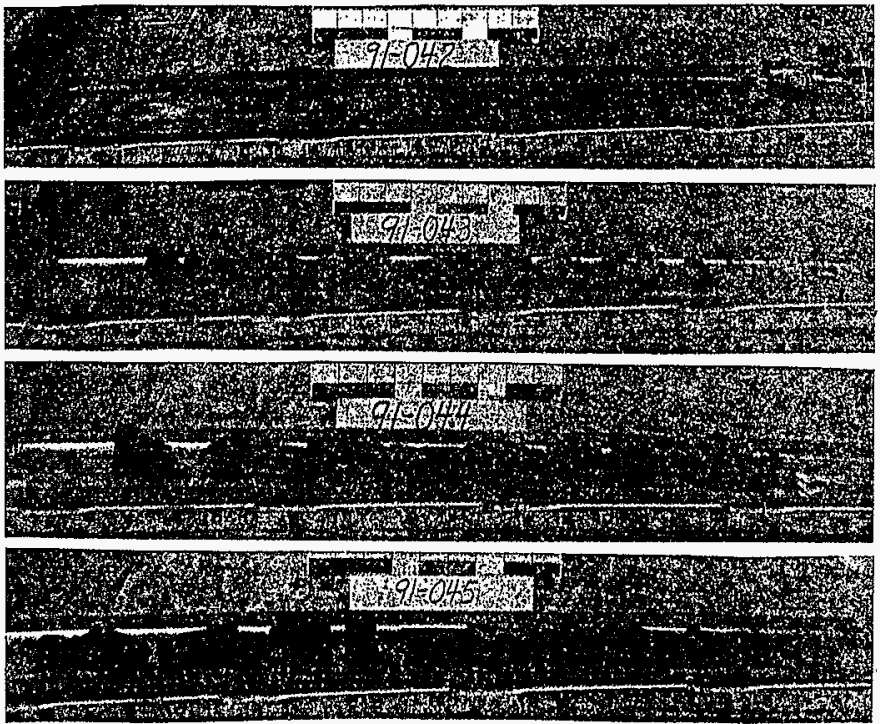

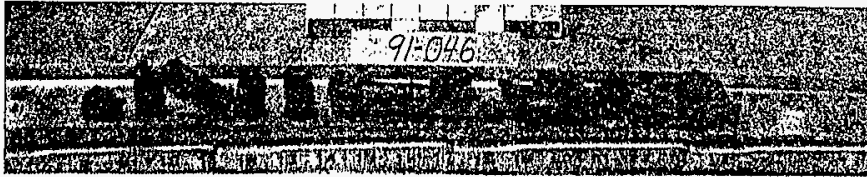
1n

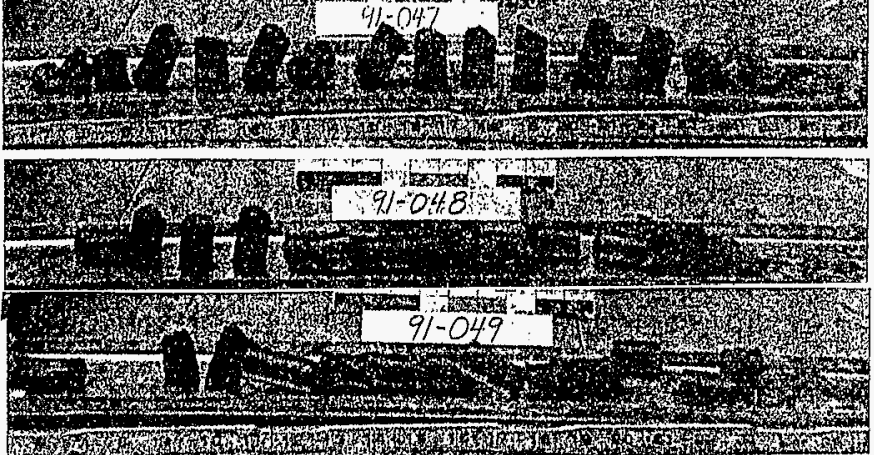


Figure 4-2. Segment Photographs for Core 27.

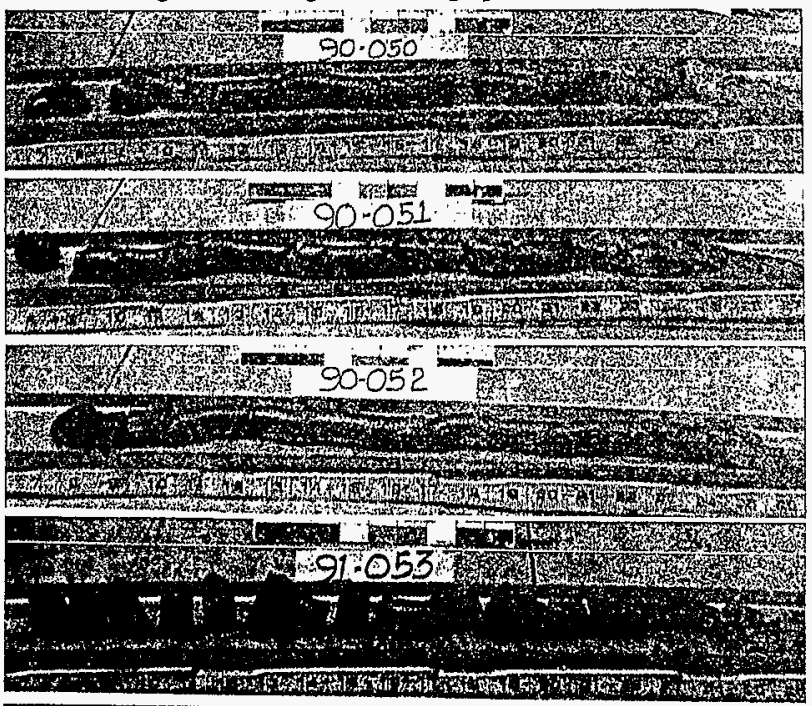

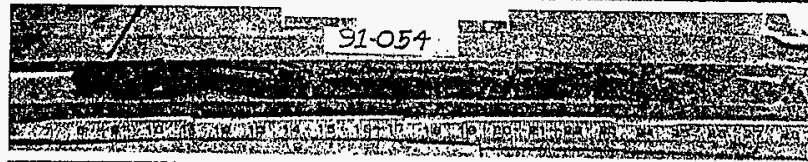
Q (3)

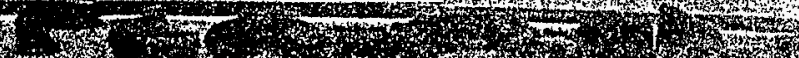
3.

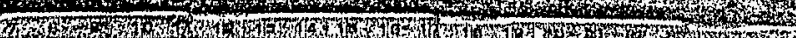

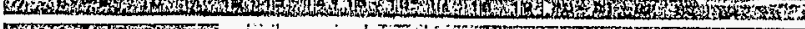
W (3) D.

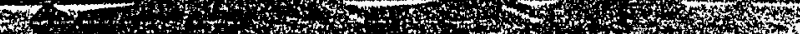

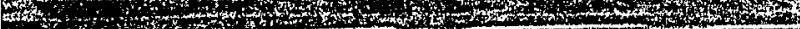

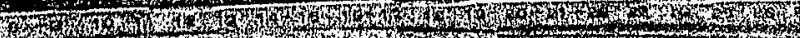

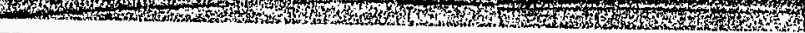

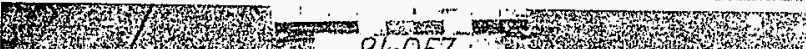
\%

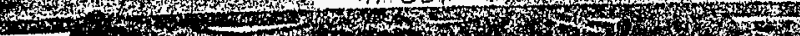

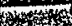
36 7 , of . M. 


\subsection{HOLDING TIME CONSIDERATIONS}

No attempt was made to meet the holding times for these samples. The samples were received from Westinghouse Hanford Company (WHC) on August 8, 1991. Analyses were not started until January, 1992. This delay was due to waste disposal issues in the 325 Building.

\subsection{SAMPLE PREPARATION AND ANALYTICAL METHODS}

Figure 4-3 contains a flowchart of the steps taken by the 325-A Laboratory to analyze tank core samples.

Figure 4-3. Sample Preparation Flowchart.
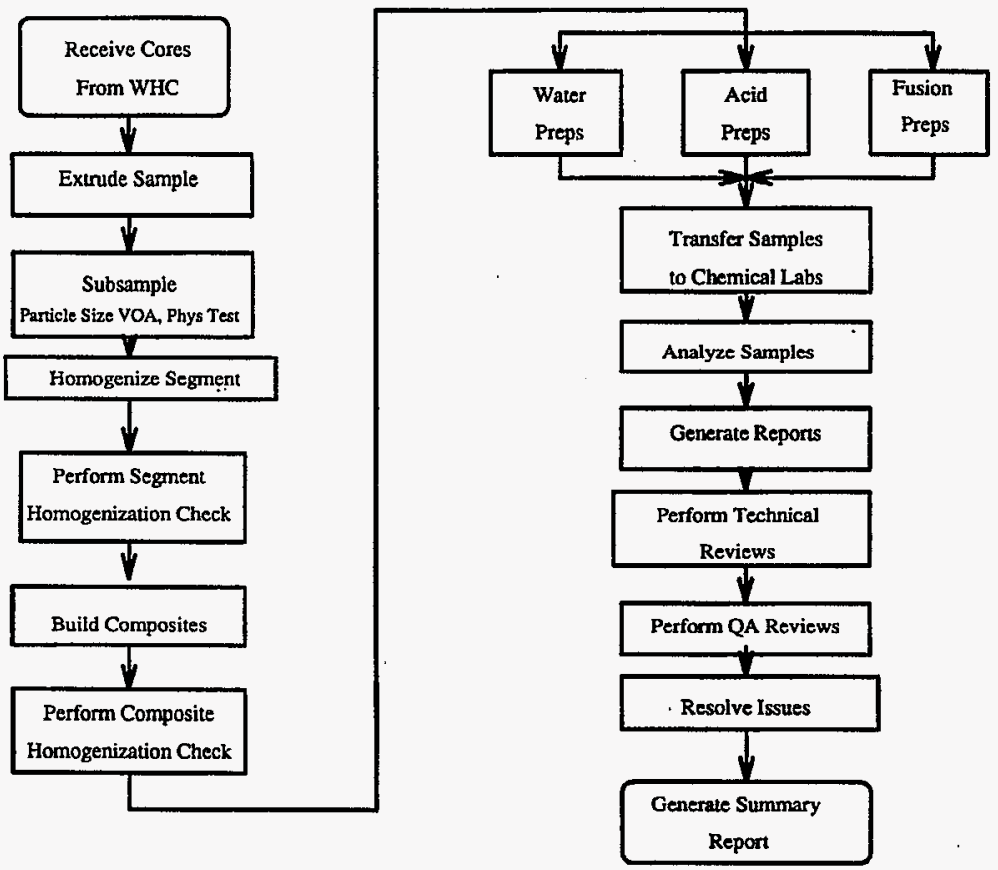
Each of the eight segments from cores 26 and 27 were homogenized. Segments 3 and 7 from core 26 and segments 3 and 6 from core 27 were subsampled for the homogenization test analyses. These subsamples were acid digested and analyzed by two methods: inductively coupled plasma atomic emission spectroscopy (ICP), and gamma energy analysis (GEA).

The core 26 relative percent differences (RPD) for the sample and duplicate within the top and bottom of each segment are significantly higher than normal, indicating either insufficient homogenization, substantial sample heterogeneity, or inadequate subsampling. In general, for both segments from core 26, the "bottom" samples have higher concentrations of most analytes than the "top" samples. This may be caused by settling prior to subsampling. Based on numerous previous homogenization tests of the Hanford tank wastes, it has been determined that fusion preparations provide more consistent and quantitative results than acid digestion; therefore, further homogenization tests (e.g., on core composites) will use fusion preparations.

Typical RPDs of 10 to $20 \%$ for many duplicate pairs are marginally acceptable; however, these high homogenization RPDs potentially compromise the accuracy of the full suite of characterization analyses performed on the core composites 1 and 2 . That is, the accuracy of any "single" analysis is biased by the inability to obtain a truly representative sampling from the blended composites. The homogenization tests are discussed in detail in Section 7.2.

In addition to these subsamples, two core composites were produced from the eight homogenized segments from each core. Homogenization test samples for the four core composites were prepared by caustic fusion and analyzed. Caustic fusion was used to prepare the samples rather than acid digestion, for reasons previously noted.

Each of the eight segments from core 27 were homogenized. Subsamples from segments 3 and 6 were prepared by acid digestion and analyzed by ICP and GEA. The homogenization test results indicated that the homogenization was insufficient. The segments were reblended and subsampled for preparation and analysis in the same manner as before. It was noted by the analyst during the preparation of the core 27 sludge that it had a greasy appearance. It was further noted that during acid digestion of the homogenization check sample, a white precipitate formed when hydrochloric acid was added. No precipitate was present while the sample was in a nitric acid media. Hydrofluoric acid and oxalic acid were added to the digestate and achieved total dissolution.

Similarly, two core composites were produced from the eight homogenized segments of core 27 . Homogenization test samples from both composites were again prepared by caustic fusion. 
The homogenized samples from cores 26 and 27 were prepared in the Shielded Analytical Laboratory (SAL), and included homogenization test samples and two core composites for each core. Due to the low level of radioactivity associated with the sludge from tank B-201, many of the analytical preparations were completed in the 325-A Laboratory (not the SAL).

Table 4-1 lists the preferred methods used to assay tank B-201 samples for the suite of requested analyses. For each analyte, the method listed as preferred produces the best estimate of concentration of that particular constituent.

Table 4-1. Sample Preparation and Analytical Methods Used on B-201 Samples. (2 pages)

\begin{tabular}{|l|l|l|l|l|l|}
\hline \multicolumn{1}{|c|}{ Analyte } & $\begin{array}{c}\text { Sample } \\
\text { Prep. }\end{array}$ & \multicolumn{1}{|c|}{ Preferred Method } & Analyte & $\begin{array}{c}\text { Sample } \\
\text { Prep. }\end{array}$ & Preferred Method \\
\hline Aluminum & A,F,W & ICP:A & Antimony & A,F,W & ICP:A \\
\hline Arsenic & A,F,W & ICP:A & Barium & A,F,W & ICP:A \\
\hline Bismuth & A,F,W & ICP:F & Beryllium & A,F,W & ICP:A \\
\hline Boron & A,F,W & ICP:A & Cadmium & A,F,W & ICP:A \\
\hline Calcium & A,F,W & ICP:A & Cerium & A,F,W & ICP:A \\
\hline Chromium & A,F,W & ICP:A & Cobalt & A,F,W & ICP:A \\
\hline Copper & A,F,W & ICP:A & Dysprosium & A,F,W & ICP:A \\
\hline Europium & A,F,W & ICP:A & Gadolinium & A,F,W & ICP:A \\
\hline Iron & A,F,W & ICP:F & Lanthanum & A,F,W & ICP:A \\
\hline Lead & A,F,W & ICP:A & Lithium & A,F,W & ICP:A \\
\hline Magnesium & A,F,W & ICP:A & Manganese & A,F,W & ICP:A \\
\hline Molybdenum & A,F,W & ICP:A & Neodymium & A,F,W & ICP:A \\
\hline Nickel & A,F,W & ICP:A & Palladium & A,F,W & ICP:A \\
\hline Phosphorus & A,F,W & ICP:F & Potassium & A,F,W & ICP:A \\
\hline Rhodium & A,F,W & ICP:A & Ruthenium & A,F,W & ICP:A \\
\hline Selenium & A,F,W & ICP:A & Silicon & A,F,W & ICP:F \\
\hline Silver & A,F,W & ICP:A & Sodium & A,F,W & ICP:F \\
\hline Strontium & A,F,W & ICP:A & Tellurium & A,F,W & ICP:A \\
\hline Thallium & A,F,W & ICP:A & Thorium & A,F,W & ICP:A \\
\hline Tin & A,F,W & ICP:A & Titanium & A,F,W & ICP:A \\
\hline Tungsten & A,F,W & ICP:A & Vanadium & A,F,W & ICP:A \\
\hline Yttrium & A,F,W & ICP:A & Zinc & A,F,W & ICP:A \\
\hline Zirconium & A,F,W & ICP:A & Sulforide & IC:W \\
\hline Cyanide & W & IC:W & W & IC:W \\
\hline Nitrate & W & IC:W & Mercury & A & IC:W \\
\hline Phosphate & W & ISE:W & WVAA:A \\
\hline Ammonia & & & IC:W \\
\hline
\end{tabular}


WHC-SD-WM-ER-550 Rev 0

Table 4-1. Sample Preparation and Analytical Methods Used on B-201 Samples. (2 pages)

\begin{tabular}{|c|c|c|c|c|c|}
\hline Analyte & $\begin{array}{c}\text { Sample } \\
\text { Prep. }\end{array}$ & Preferred Method & Analyte & $\begin{array}{c}\text { Sample } \\
\text { Prep. }\end{array}$ & Preferred Method \\
\hline Curium-243/244 & $F$ & Alpha Radchem:F & Gross alpha & $\bar{F}$ & Alpha Radchem:F \\
\hline Neptunium-237 & $\mathrm{F}$ & Alpha Radchem:F & Plutonium-238 & $F$ & Alpha Radchem:F \\
\hline Plutonium-239/240 & $\mathrm{F}$ & Alpha Radchem:F & Total alpha & $\mathrm{F}, \mathrm{W}$ & Alpha Radchem:F \\
\hline Gross beta & $\mathbf{F , W}$ & Beta Radchem:F & Strontium-90 & $\mathbf{F}$ & Beta Radchem:F \\
\hline Technetium-99 & $\mathrm{F}$ & Beta Radchem:F & Americium-241 & A,F,W & GEA:F \\
\hline Cerium-144 & $A, F, W$ & GEA:F & Cesium-134 & $\mathbf{A}, \mathbf{F}, \mathbf{W}$ & GEA:F \\
\hline Cesium-137 & A,F,W & GEA:F & Cobalt-60 & $\mathbf{A}, \mathbf{F}, \mathbf{W}$ & GEA:F \\
\hline Europium-154 & $\mathrm{A}, \mathrm{F}, \mathrm{W}$ & GEA:F & Europium-155 & $\mathbf{A}, \mathbf{F}, \mathbf{W}$ & GEA:F \\
\hline Potassium -40 & $A, F, W$ & GEA:F & Uranium & $\mathrm{F}$ & Laser Fluorimetry:F \\
\hline Plutonium-239 & $\mathrm{p}$ & Mass Spectrometry:F & Plutonium-240 & $F$ & Mass Spectrometry:F \\
\hline Plutonium-241 & $\mathbf{F}$ & Mass Spectrometry:F & Plutonium-242 & $\mathbf{F}$ & Mass Spectrometry:F \\
\hline Uranium-234 & F & Mass Spectrometry:F & Uranium-235 & F & Mass Spectrometry:F \\
\hline Uranium-236 & $\mathrm{F}$ & Mass Spectrometry:F & Uranium-238 & $\mathbf{F}$ & Mass Spectrometry:F \\
\hline Tritium & $\bar{W}$ & Liq Scintillation:W & Carbon-14 & & Liq Scintillation:W \\
\hline Nickel-59 & A & Liq Scintillation:A & Nickel-63 & A & Beta Radchem:F \\
\hline TOC & $\mathrm{D}, \mathrm{W}$ & Persulfate Oxidation:D & Hex. Chromium & $\mathbf{w}$ & Calorimetric:W \\
\hline Total carbon & $\mathrm{D}, \mathrm{W}$ & Persulfate Oxidation:W & TIC & $\mathbf{D}, \mathbf{W}$ & Persulfate Oxidation: $W$ \\
\hline SVOA & & GC/Mass Spectrometry & VOA & & GC/Mass Spectrometry \\
\hline
\end{tabular}

A: acid digestion; CVAA: cold vapor atomic absorption; D: direct analysis; F: KOH/Ni fusion; GC: gas chromatography; ISE: ion-specific electrode; SVOA: semi-volatile organics analysis; VOA: volatile organics analysis; W: water digestion.

Caustic fusion, acid digestion, and water leach preparations of all core composites were completed in the SAL. Tests requiring little or no sample preparation - such as weight percent solids, direct total carbon, direct total inorganic carbon (TIC), direct total organic carbon (TOC), carbon-14, and pH - were conducted in-cell. Due to the low level of radioactivity, aliquots were provided directly to the 325-A Laboratory for mercury, toxicity characteristic leach procedure (TCLP), semivolatile organic analysis, and extractable organic halides analysis.

The SAL made deliberate minor deviations to sample preparation procedures for one or more of the following reasons:

Insufficient sample was available to conduct the analyses per the specified procedure, and still maintain the level of quality control requested.

- Sample weights and/or final volumes were reduced to facilitate waste minimization. 
- Sample weights and/or final volumes were altered to increase the concentration of certain analytes of interest. This was done to meet the concentration ranges needed to perform the analyses, as specified in the procedures.

These deviations are not expected to have a substantive impact on the analytical results or on any conclusions derived from them. 


\subsection{ANALYTICAL RESULTS AND WASTE INVENTORY}

A total of 7,598 analytical measurements were made on tank B-201, and Table 5-1 contains a summary of the analytical result counts. As shown, the most complete segmentlevel analyses were performed on physical properties. The majority of the segment-level chemical analyses were homogenization tests. The only exception is for segment 1 of core 26 , which was analyzed for volatile organics. Nearly $33 \%$ of all analytical results in the B-201 dataset are quality assurance (QA) data (i.e., matrix spikes, method blanks, etc.). If the homogenization test data is included as QA data, this percentage goes up to $59 \%$ (i.e., more than one-half of the analytical results in the B-201 dataset were taken for QA reasons).

Table 5-1. Summary of B-201 Analytical Result Counts.

\begin{tabular}{|l|l|rrrrrrrr|r|}
\hline \multicolumn{1}{|c}{} & $\mathbf{1}$ & $\mathbf{2}$ & $\mathbf{3}$ & $\mathbf{4}$ & $\mathbf{5}$ & $\mathbf{6}$ & 7 & $\mathbf{8}$ & Composite \\
\hline \multirow{2}{*}{$\begin{array}{l}\text { Physical } \\
\text { Properties }\end{array}$} & Core 26 & 8 & 43 & 7 & 5 & 43 & 5 & 5 & 43 & 8 \\
\cline { 2 - 12 } & Core 27 & 27 & 27 & 15 & 27 & 27 & 23 & 19 & 19 & 0 \\
\hline \multirow{2}{*}{$\begin{array}{l}\text { Chemical } \\
\text { Analyses }\end{array}$} & Core 26 & 74 & 0 & 283 & 0 & 0 & 0 & 274 & 0 & 1,682 \\
\cline { 2 - 12 } & Core 27 & 0 & 0 & 404 & 0 & 0 & 400 & 0 & 0 & 1,641 \\
\hline \multicolumn{2}{|c|}{ QA Data } & 108 & 0 & 231 & 0 & 0 & 100 & 134 & 0 & 1,907 \\
\hline \multicolumn{2}{|c|}{ Totals } & 217 & 70 & 940 & 32 & 70 & 528 & 432 & 62 & 5,238 \\
\hline
\end{tabular}

The core composite data was used primarily to determine mean concentrations and their associated uncertainties, for the inventory of tank B-201. The segment-level data were used, however, for the analysis of physical properties. A summary of the results from the statistical analysis are given in this section. The complete results are contained in Appendixes B and $\mathrm{C}$.

\subsection{CHEMICAL ANALYSES AND RADIOLOGICAL DETERMINATIONS}

Due to the sampling structure in the B-201 composite data, the following random effects model was fit for each constituent:

$$
Y_{i j k}=\mu+C_{i}+S_{i j}+E_{i j k}
$$


where:

$Y_{i j k}=$ The measured value of concentration of a constituent in composite $j$ of core $i$

$\mu=$ The mean concentration of the constituent

$C_{i}=$ The deviation of core $i$ from the mean (i.e., horizontal variability)

$S_{i j}=$ The deviation of composite $j$ within core $i$ from the mean

$E_{i j k}=$ Represents sampling and analytical deviations.

As can be seen, each term in the model describes the contribution of each step in the sampling and measurement process to the observed analytical value (e.g., its calculated mean and the components of variability). For each constituent, this model can be used to obtain a mean concentration estimate, along with its associated uncertainty. This model can also be used to obtain estimates of horizontal variability $C_{i}$, sampling variability $S_{i}$, and analytical variability $E_{i j k}$ for each constituent.

Table 5-2 lists the tank inventories and concentrations calculated from this analysis of variance (ANOVA) model. Some of the constituents shown in this table were analyzed by more than one method, but only the results from the preferred analytical method are presented. The complete set of constituent results is contained in Appendix B. The historical estimates listed in Table 5-2 are extracted from Table 6-4. The total inventories are calculated using an assumed density of $1.25 \mathrm{~g} / \mathrm{ml}$ and a total volume of 109,777 liters (29,000 gallons).

Table 5-2. Summary of the Composite Level Results for Anions, Metals, Organics, and Radionuclides. (6 pages)

\begin{tabular}{|l|l|r|r|r|r|r|}
\hline \multirow{2}{*}{ Analyte } & \multirow{2}{*}{$\begin{array}{l}\text { Analytical Method: } \\
\text { Sample Preparation }\end{array}$} & \multicolumn{3}{c|}{ Mean Concentration } & Total \\
Inventory
\end{tabular}


Table 5-2. Summary of the Composite Level Results for Anions, Metals, Organics, and Radionuclides. (6 pages)

\begin{tabular}{|c|c|c|c|c|c|}
\hline \multirow{2}{*}{ Analyte } & \multirow{2}{*}{$\begin{array}{l}\text { Analytical Method: } \\
\text { Sample Preparation }\end{array}$} & \multicolumn{3}{|c|}{ Mean Concentration } & \multirow{2}{*}{$\begin{array}{c}\text { Total } \\
\text { Inventory }\end{array}$} \\
\hline & & Composite & RSD & LANL & \\
\hline \multicolumn{6}{|c|}{ Cations } \\
\hline & & $(\mu \mathrm{g} / \mathrm{g})$ & & $(\mu \mathrm{g} / \mathrm{g})$ & $\overline{(\mathrm{kg})}$ \\
\hline Aluminum & ICP:A & $3.44 e+03$ & 74 & NA & $4.72 e+02$ \\
\hline Ammonia & ISE:W & $1.04 e+01$ & 46 & NA & $1.43 e+00$ \\
\hline Antimony & ICP:A & $<3.71 \mathrm{e}+01$ & NA & NA & $<5.09 \mathrm{e}+00$ \\
\hline Arsenic & ICP:A & $5.95 \mathrm{e}+01$ & NA & NA & $8.16 e+00$ \\
\hline Barium & ICP:A & $8.64 \mathrm{e}+01$ & 38 & NA & $1.19 e+01$ \\
\hline Beryllium & ICP:A & $<3.71 \mathrm{e}+00$ & NA & NA & $<5.09 e-01$ \\
\hline Bismuth & ICP:F & $9.45 e+04$ & 3 & $2.96 e+04$ & $1.30 e+04$ \\
\hline Boron & ICP:A & $7.05 \mathrm{e}+01$ & 35 & NA & $9.67 \mathrm{e}+00$ \\
\hline Cadmium & ICP:A & $4.81 \mathrm{e}+00$ & 6 & NA & $6.60 \mathrm{e}-01$ \\
\hline Calcium & ICP:A & $1.22 \mathrm{e}+04$ & 58 & $0.00 c+00$ & $1.67 e+03$ \\
\hline Cerium & ICP:A & $6.96 e+01$ & 11 & NA & $9.55 e+00$ \\
\hline Chromium & ICP:A & $3.34 e+03$ & 4 & $1.07 e+03$ & $4.58 \mathrm{e}+02$ \\
\hline Cobait & ICP:A & $9.60 \mathrm{e}+00$ & 4 & NA & $1.32 \mathrm{e}+00$ \\
\hline Copper & ICP:A & $4.82 \mathrm{e}+01$ & 67 & NA & $6.61 e+00$ \\
\hline Dysprosium & ICP:A & $<2.94 \mathrm{e}+01$ & 31 & NA & $<4.03 e+00$ \\
\hline Europium & ICP:A & $<7.43 \mathrm{e}+00$ & NA & NA & $<1.02 \mathrm{e}+00$ \\
\hline Gadolinium & ICP:A & $1.69 e+02$ & 15 & NA & $2.32 e+01$ \\
\hline Hexavalent Chromium & Calorimetric:W & $7.47 e+02$ & 6 & NA & $1.03 e+02$ \\
\hline Iron & ICP;F & $1.34 \mathrm{e}+04$ & 20 & $\mathrm{NA}$ & $1.84 e+03$ \\
\hline Lanthanum & ICP:A & $1.51 \mathrm{e}+04$ & 10 & NA & $2.07 e+03$ \\
\hline Lead & ICP:A & $1.36 e+03$ & 9 & NA & $1.87 e+02$ \\
\hline Lithium & ICP:A & $<1.49 e+01$ & NA & NA & $<2.04 \mathrm{e}+00$ \\
\hline Magnesium & ICP:A & $1.51 e+03$ & 58 & NA & $2.07 e+02$ \\
\hline Manganese & ICP:A & $1.92 \mathrm{e}+04$ & 29 & $5.77 e+03$ & $2.63 e+03$ \\
\hline Mercury & CVAA:A & $5.99 \mathrm{e}-01$ & 46 & NA & $8.22 \mathrm{e}-02$ \\
\hline Molybdenum & ICP:A & $1.91 \mathrm{e}+01$ & 4 & NA & $2.62 e+00$ \\
\hline Neodymium & ICP:A & $<2.23 \mathrm{e}+01$ & NA & NA & $<3.06 \mathrm{e}+00$ \\
\hline Nickel & ICP:A & $4.79 e+02$ & 3 & NA & $6.57 e+01$ \\
\hline Palladium & ICP:A & $<1.11 \mathrm{e}+02$ & NA & NA & $<1.52 \mathrm{e}+01$ \\
\hline Phosphorus & ICP:F & $5.45 e+03$ & 14 & NA & $7.48 \mathrm{e}+02$ \\
\hline Potassium & ICP:A & $5.81 e+03$ & 13 & $9.01 e+03$ & $7.97 e+02$ \\
\hline Rhodium & ICP:A & $<7.43 e+01$ & NA & NA & $<1.02 \mathrm{e}+01$ \\
\hline Ruthenium & ICP:A & $<3.71 \mathrm{e}+01$ & NA & NA & $<5.09 e+00$ \\
\hline
\end{tabular}


Table 5-2. Summary of the Composite Level Results for Anions, Metals, Organics, and Radionuclides. (6 pages)

\begin{tabular}{|c|c|c|c|c|c|}
\hline \multirow{2}{*}{ Analyte } & \multirow{2}{*}{$\begin{array}{l}\text { Analytical Method: } \\
\text { Sample Preparation }\end{array}$} & \multicolumn{3}{|c|}{ Mean Concentration } & \multirow{2}{*}{$\begin{array}{c}\text { Total } \\
\text { Inventory }\end{array}$} \\
\hline & & Composite & RSD & LANL & \\
\hline Selenium & ICP:A & $6.68 \mathrm{e}+01$ & 9 & NA & $9.17 e+00$ \\
\hline Silicon & ICP:F & $2.02 \mathrm{e}+04$ & 63 & NA & $2.77 \mathrm{e}+03$ \\
\hline Silver & ICP:A & $1.23 \mathrm{e}+01$ & 20 & NA & $1.69 e+00$ \\
\hline Sodium & ICP:F & $3.82 \mathrm{e}+04$ & 2 & $3.90 \mathrm{e}+0.4$ & $5.24 e+03$ \\
\hline Strontium & ICP:A & $9.23 \mathrm{e}+02$ & 5 & NA & $1.27 \mathrm{e}+02$ \\
\hline Tellurium & ICP:A & $<7.43 \mathrm{e}+01$ & NA & NA & $<1.02 \mathrm{e}+01$ \\
\hline Thallium & ICP:A & $<3.71 e+02$ & $\overline{\mathbf{N A}}$ & NA & $<5.09 \mathrm{e}+01$ \\
\hline Tin & ICP:A & $5.96 e+02$ & NA & NA & $8.18 \mathrm{e}+01$ \\
\hline Titanium & ICP:A & $2.85 e+02$ & 75 & NA & $3.91 \mathrm{e}+01$ \\
\hline Tungsten & ICP:A & $5.96 \mathrm{e}+01$ & NA & NA & $8.18 e+00$ \\
\hline Uranium & Laser Fluorimetry:F & $1.56 \mathrm{e}+02$ & 100 & $0.00 \mathrm{e}+00$ & $2.14 e+01$ \\
\hline Vanadium & ICP:A & $1.59 e+01$ & 38 & NA & $2.18 \mathrm{e}+00$ \\
\hline Yttrium & ICP:A & $8.19 \mathrm{e}+00$ & 18 & NA & $1.12 \mathrm{e}+00$ \\
\hline Zine & ICP:A & $2.17 e+02$ & 6 & NA & $2.98 \mathrm{e}+01$ \\
\hline Zirconium & ICP:A & $1.07 \mathrm{e}+01$ & 10 & NA & $1.47 e+00$ \\
\hline \multicolumn{6}{|c|}{ Organies } \\
\hline & & $(\mu \mathrm{g} / \mathrm{g})$ & & $(\mu \mathrm{g} / \mathrm{g})$ & (kg) \\
\hline 1,2,4-Trichlorobenzene & SVOA & $<4.80 e+01$ & NA & NA & $<6.59 \mathrm{e}+00$ \\
\hline 1,2-Dichlorobenzene & SVOA & $<4.80 \mathrm{e}+01$ & NA & NA & $<6.59 \mathrm{e}+00$ \\
\hline 1,3-Dichlorobenzene & SVOA & $<4.80 \mathrm{e}+01$ & NA & NA & $<6.59 \mathrm{e}+00$ \\
\hline 1,4-Dichlorobenzene & SVOA & $<4.80 e+01$ & NA & NA & $<6.59 \mathrm{e}+00$ \\
\hline 2,4,5-Trichlorophenol & SVOA & $<2.40 \mathrm{e}+02$ & NA & NA & $<3.29 \mathrm{e}+01$ \\
\hline 2,4,6-Trichlorophenol & SVOA & $<4.80 \mathrm{e}+01$ & NA & NA & $<6.59 e+00$ \\
\hline 2,4-Dichlorophenol & SVOA & $<4.80 \mathrm{e}+01$ & NA & NA & $<6.59 \mathrm{e}+00$ \\
\hline 2,4-Dimethylphenol & SVOA & $<4.80 e+01$ & NA & NA & $<6.59 \mathrm{e}+00$ \\
\hline 2,4-Dinitrophenol & SVOA- & $<2.40 \mathrm{e}+02$ & NA & NA & $<3.29 \mathrm{e}+01$ \\
\hline 2,4-Dinitrotoluene & SVOA & $<4.80 \mathrm{e}+01$ & NA & NA & $<6.59 \mathrm{e}+00$ \\
\hline 2,6-Dinitrotoluene & SVOA & $<4.80 e+01$ & NA & NA & $<6.59 \mathrm{e}+00$ \\
\hline 2-Chloronaphthalene & SVOA & $<4.80 \mathrm{e}+01$ & NA & NA & $<6.59 \mathrm{e}+00$ \\
\hline 2-Chlorophenol & SVOA & $<4.80 e+01$ & NA & NA & $<6.59 \mathrm{e}+00$ \\
\hline 2-Methylnaphthalene & SVOA & $<4.80 e+01$ & NA & $\overline{N A}$ & $<6.59 e+00$ \\
\hline 2-Methylphenol & SVOA & $<4.80 \mathrm{e}+01$ & NA & NA & $<6.59 c+00$ \\
\hline 2-Nitroaniline & SVOA & $<2.40 e+02$ & NA & NA & $<3.29 \mathrm{e}+01$ \\
\hline 2-Nitrophenol & SVOA & $<4.80 e+01$ & NA & NA & $<6.59 \mathrm{e}+00$ \\
\hline 3,3'-Dichlorobenzidine & SVOA & $<9.69 \mathrm{e}+01$ & NA & NA & $<1.33 \mathrm{e}+01$ \\
\hline 3-Nitroaniline & SVOA & $<2.40 e+02$ & NA & NA & $<3.29 \mathrm{e}+01$ \\
\hline 4,6-Dinitro-o-cresol & SVOA & $<2.40 \mathrm{e}+02$ & $\overline{N A}$ & $\overline{\mathrm{NA}}$ & $<3.29 \mathrm{e}+01$ \\
\hline
\end{tabular}


Table 5-2. Summary of the Composite Level Results for Anions, Metals, Organics, and Radionuclides. (6 pages)

\begin{tabular}{|c|c|c|c|c|c|}
\hline \multirow{2}{*}{ Analyte } & \multirow{2}{*}{$\begin{array}{l}\text { Analytical Method: } \\
\text { Sample Preparation }\end{array}$} & \multicolumn{3}{|c|}{ Mean Concentration } & \multirow{2}{*}{$\begin{array}{c}\text { Total } \\
\text { Inventory }\end{array}$} \\
\hline & & Composite & RSD & LANL & \\
\hline 4-Bromophenylphenyl ether & SVOA & $<4.80 \mathrm{e}+01$ & NA & NA & $<6.59 \mathrm{e}+00$ \\
\hline 4-Chloro-3-methylphenol & SVOA & $<4.80 \mathrm{e}+01$ & NA & NA & $<6.59 \mathrm{e}+00$ \\
\hline 4-Chloroaniline & SVOA & $<4.80 \mathrm{e}+01$ & NA & NA & $<6.59 \mathrm{e}+00$ \\
\hline 4-Methylphenol & SVOA & $<4.800+01$ & NA & NA & $<6.59 \mathrm{e}+00$ \\
\hline 4-Nitroaniline & SVOA & $<2.40 c+02$ & NA & NA & $<3.29 \mathrm{e}+01$ \\
\hline 4-Nitrophenol & SVOA & $<2.40 \mathrm{e}+02$ & NA & NA & $<3.29 \mathrm{e}+01$ \\
\hline Acenaphthene & SVOA & $<4.80 \mathrm{e}+01$ & NA & NA & $<6.59 e+00$ \\
\hline Acenaphthylene & SVOA & $<4.80 e+01$ & NA & NA & $<6.59 \mathrm{e}+00$ \\
\hline Anthracene & SVOA & $<4.80 \mathrm{e}+01$ & NA & NA & $<6.59 \mathrm{e}+00$ \\
\hline Benzo(a)anthracene & SVOA & $<4.80 e+01$ & NA & NA & $<6.59 \mathrm{e}+00$ \\
\hline Benzo(a)pyrene & SVOA & $<4.80 \mathrm{e}+01$ & NA & NA & $<6.59 \mathrm{e}+00$ \\
\hline Benzo(b)fluoranthene & SVOA & $<4.80 \mathrm{e}+01$ & NA & NA & $<6.59 \mathrm{e}+00$ \\
\hline Benzo(ghi)perylene & SVOA & $<4.80 c+01$ & NA & NA & $<6.59 \mathrm{e}+00$ \\
\hline Benzo(k)fluoranthene & SVOA & $<4.80 e+01$ & NA & NA & $<6.59 e+00$ \\
\hline Benzoic acid & SVOA & $<2.40 \mathrm{e}+02$ & NA & NA & $<3.29 e+01$ \\
\hline Benzyl alcohol & SVOA & $<4.800+01$ & NA & NA & $<6.59 e+00$ \\
\hline Bis(2-chloroethyl) ether & SVOA & $<4.80 \mathrm{e}+01$ & NA & NA & $<6.59 \mathrm{e}+00$ \\
\hline Bis(2-chloroisopropyI) & SVOA & $<4.80 \mathrm{e}+01$ & NA & NA & $<6.59 \mathrm{e}+00$ \\
\hline Butylbenzylphthalate & SVOA & $<4.80 \mathrm{e}+01$ & NA & NA & $<6.59 \mathrm{e}+00$ \\
\hline Chrysene & SVOA & $<4.80 e+01$ & NA & NA & $<6.59 e+00$ \\
\hline Di-n-butylphthalate & SVOA & $<4.80 \mathrm{e}+01$ & NA & NA & $<6.59 \mathrm{e}+00$ \\
\hline Di-n-octylphthalate & SVOA & $<4.80 \mathrm{e}+01$ & NA & NA & $<6.59 \mathrm{e}+00$ \\
\hline Dibenz $[a, h]$ anthracene & SVOA & $<4.80 \mathrm{e}+01$ & NA & NA & $<6.59 e+00$ \\
\hline Dibenzofuran & SVOA & $<4.80 \mathrm{e}+01$ & NA & NA & $<6.59 \mathrm{e}+00$ \\
\hline Diethylphthalate & SVOA & $<4.80 \mathrm{e}+01$ & NA & NA & $<6.59 e+00$ \\
\hline Dimethyl phthalate & SVOA & $<4.80 \mathrm{e}+01$ & NA & NA & $<6.59 e+00$ \\
\hline Dodecane & SVOA & $2.85 e+02$ & 8 & NA & $3.91 e+01$ \\
\hline Fluoranthene & SVOA & $<4.80 \mathrm{e}+01$ & NA & NA & $<6.59 \mathrm{e}+00$ \\
\hline Fluorene & SVOA & $<4.80 \mathrm{e}+01$ & NA & NA & $<6.59 e+00$ \\
\hline Hexachlorobenzene & SVOA & $<4.80 e+01$ & NA & NA & $<6.59 e+00$ \\
\hline Hexachlorobutadiene & SVOA & $<4.80 \mathrm{e}+01$ & NA & NA & $<6.59 \mathrm{e}+00$ \\
\hline Hexachloroethane & SVOA & $<4.80 \mathrm{e}+01$ & NA & NA & $<6.59 e+00$ \\
\hline Indeno( $(1,2,3-c d)$ pyrene & SVOA & $<4,80 \mathrm{e}+01$ & NA & NA & $<6.59 \mathrm{e}+00$ \\
\hline Isophorone & SVOA & $<4.80 \mathrm{e}+01$ & NA & NA & $<6.59 e+00$ \\
\hline N-Nitrosodiphenylamine & SVOA & $<4.80 \mathrm{e}+01$ & NA & NA & $<6.59 e+00$ \\
\hline Naphthalene & SVOA & $<4.80 \mathrm{e}+01$ & NA & NA & $<6.59 \mathrm{e}+00$ \\
\hline Nitrobenzene & SYOA & $<4.80 \mathrm{e}+01$ & NA & NA & $<6.59 e+00$ \\
\hline
\end{tabular}


Table 5-2. Summary of the Composite Level Results for Anions, Metals, Organics, and Radionuclides. (6 pages)

\begin{tabular}{|c|c|c|c|c|c|}
\hline \multirow{2}{*}{ Analyte } & \multirow{2}{*}{$\begin{array}{l}\text { Analytical Method: } \\
\text { Sample Preparation }\end{array}$} & \multicolumn{3}{|c|}{ Mean Concentration } & \multirow{2}{*}{$\begin{array}{c}\text { Total } \\
\text { Inventory }\end{array}$} \\
\hline & & Composite & RSD & LANL & \\
\hline Pentachlorophenol & SVOA & $<2.40 \mathrm{e}+02$ & NA & NA & $<3.29 \mathrm{e}+01$ \\
\hline Pentadecane & SVOA & $4.10 \mathrm{e}+01$ & 37 & NA & $5.63 e+00$ \\
\hline Phenanthrene & SVOA & $<4.80 \mathrm{e}+01$ & NA & NA & $<6.59 \mathrm{e}+00$ \\
\hline Phenol & SVOA & $<4.80 e+01$ & $\mathbf{N A}$ & NA & $<6.59 e+00$ \\
\hline Pyrene & SVOA & $<4.80 \mathrm{e}+01$ & NA & NA & $<6.59 \mathrm{e}+00$ \\
\hline Tetradecane & SVOA & $1.03 e+03$ & 28 & NA & $1.41 \mathrm{e}+02$ \\
\hline Total carbon & Persulfate Oxidation:W & $2.55 e+03$ & 12 & NA & $3.50 e+02$ \\
\hline Total inorganic carbon & Persulfate Oxidation:W & $2.09 e+03$ & 16 & $\overline{N A}$ & $2.87 e+02$ \\
\hline Total organic carbon & Persulfate Oxidation:W & $5.18 \mathrm{e}+02$ & 10 & $4.58 \mathrm{e}+03$ & $7.11 \mathrm{e}+01$ \\
\hline Tridecane & SVOA & $9.29 \mathrm{e}+02$ & 7 & NA & $1.27 e+02$ \\
\hline \multicolumn{6}{|c|}{ Physical Properties } \\
\hline & & (\%) & & (\%) & \\
\hline Weight percent solids & Percent Solid & $3.93 e+01$ & 3 & NA & NA \\
\hline \multicolumn{6}{|c|}{ Radionuclides } \\
\hline & & $(\mu \mathrm{Ci} / \mathrm{g})$ & & $(\mu \mathrm{Ci} / \mathrm{g})$ & (Ci) \\
\hline Americium-241 & GEA:F & $3.10 \mathrm{e}-02$ & 4 & NA & $4.25 e+00$ \\
\hline Carbon-14 & Liq Scintillation:W & $3.16 \mathrm{e}-04$ & NA & NA & $4.34 e-02$ \\
\hline Cesium-134 & GBA:F & $2.38 \mathrm{e}-03$ & NA & NA & $3.27 e-01$ \\
\hline Cesium-137 & GEA:F & $8.000-01$ & 27 & NA & $1.10 c+02$ \\
\hline Cobalt-60 & GEA:F & $1.96 \mathrm{e}-03$ & 75 & NA & $2.69 \mathrm{e}-01$ \\
\hline Curium-243/244 & Alpha Radchem:F & $1.64 \mathrm{e}-03$ & 21 & NA & $2.25 \mathrm{e}-01$ \\
\hline Europium-154 & GEA:F & $4.38 e-03$ & 51 & NA & $6.01 e-01$ \\
\hline Europium-155 & GEA:F & $3.28 \mathrm{e}-03$ & NA & NA & $4.50 \mathrm{e}-01$ \\
\hline Gross alpha & Alpha Radchem:F & $1.31 \mathrm{e}+00$ & 18 & NA & $1.80 e+02$ \\
\hline Gross beta & Beta Radchem:F & $4.41 \mathrm{e}+00$ & 28 & $\mathrm{NA}$ & $6.05 e+02$ \\
\hline Neptunium-237 & Alpha Radchem:F & $<1.24 \mathrm{e}-04$ & NA & NA & $<1.70 \mathrm{e}-02$ \\
\hline Nickel-59 & Beta Radchem:A & $6.86 \mathrm{e}-06$ & 22 & NA & $9.41 e-04$ \\
\hline Nickel-63 & Liq Scintillation:A & $1.88 \mathrm{e}-04$ & 25 & $\mathbf{N A}$ & $2.58 \mathrm{e}-02$ \\
\hline Plutonium-238 & Alpha Radchem:F & $3.48 \mathrm{e}-03$ & 98 & $\widehat{N A}$ & $4.78 e-01$ \\
\hline Plutonium-239/240 & Alpha Radchem:F & $1.13 e+00$ & 30 & NA & $1.55 c+02$ \\
\hline Strontium-90 & Beta Radchem:F & $2.09 e+00$ & 51 & $0.00 e+03$ & $2.87 e+02$ \\
\hline Technetium-99 & Beta Radchem:F & $<1.94 \mathrm{e}-03$ & NA & NA & $<2.66 \mathrm{e}-01$ \\
\hline Total alpha* & Alpha Radchem:F & $1.14 \mathrm{e}+00$ & NA & NA & $1.56 \mathrm{e}+02$ \\
\hline Tritium & Liq Scintillation:W & $2.05 e-02$ & 78 & NA & $2.81 e+00$ \\
\hline
\end{tabular}


Table 5-2. Summary of the Composite Level Results for Anions, Metals, Organics, and Radionuclides. (6 pages)

\begin{tabular}{|c|c|c|c|c|c|}
\hline \multirow{2}{*}{ Analyte } & \multirow{2}{*}{$\begin{array}{l}\text { Analytical Method: } \\
\text { Sample Preparation }\end{array}$} & \multicolumn{3}{|c|}{ Mean Concentration } & \multirow{2}{*}{$\begin{array}{c}\text { Total } \\
\text { Inventory }\end{array}$} \\
\hline & & Composite & RSD & LANL & \\
\hline & & (\%) & & $(\%)$ & \\
\hline Uranium-234 & Mass Spectrometry:F & $5.40 \mathrm{e}-03$ & 6 & NA & NA \\
\hline Uranium-235 & Mass Spectrometry:F & $6.91 e-01$ & 0 & NA & NA \\
\hline Uranium-236 & Mass Spectrometry:F & $5.20 \mathrm{e}-03$ & 6 & NA & $\overline{\text { NA }}$ \\
\hline Uranium-238 & Mass Spectrometry:F & $9.93 e+01$ & 0 & NA & $\overline{\mathrm{NA}}$ \\
\hline Plutonium-239 & Mass Spectrometry: $\mathrm{F}$ & $9.84 \mathrm{e}+01$ & 0 & $\overline{\text { NA }}$ & NA \\
\hline Plutonium-240 & Mass Spectrometry:F & $1.55 e+00$ & 1 & NA & NA \\
\hline Plutonium-241 & Mass Spectrometry:F & $1.30 \mathrm{e}-02$ & 16 & NA & NA \\
\hline Plutonium-242 & Mass Spectrometry:F & $3.84 \mathrm{e}-03$ & 43 & NA & NA \\
\hline
\end{tabular}

*Total alpha emitted from ${ }^{238} \mathrm{Pu},{ }^{239} \mathrm{Pu},{ }^{240} \mathrm{Pu},{ }^{201} \mathrm{Pu}$; NA: Not Available or Not Applicable.

Table 5-2 shows each constituent's mean concentration and relative standard deviation (RSD) on the mean. The RSD is the square root of the variance estimate divided by the mean of the constituent, which indicates how large the variance estimate is relative to the mean. If a sample result was below the detection limit, the detection limit was used in the fit of the random effects model. If more than $75 \%$ of the sample results for a given constituent were below the detection limit, the random effects model was not fit. In that case, a mean was taken and no RSD information was reported.

The plutonium analyses done for core 26 and core 27 were not identical. In the case of core 26 , the alpha contributors were chemically separated, and an alpha energy analysis was done. This assay provided the concentrations of Plutonium-238, Plutonium-239/240, and Americium-241. In the case of core 27, a total alpha measurement was taken and the contributors from the various alpha emitters resolved. The sample was then chemically prepared and assayed with a mass spectrometer to determine the isotopic content of the plutonium in the sample. In order to provide a representative estimate of the plutonium inventory for tank B-201, the information in core 27 about plutonium content and isotopic distribution was used to determine the corresponding values found in the core 26 measurements and included in the ANOVA analyses.

The boxplots in Figure 5-1 illustrate the magnitude of horizontal, sampling, and analytical variance components relative to each other. The "box" for a given boxplot represents the range of the middle $50 \%$ of the RSDs. The vertical line in each box is the median RSD value and the lines (whiskers) coming out the ends of the boxes represent the entire range of the RSDs. For all subgroupings of constituents (anions, cations or metals, organics, radionuclides), the horizontal spatial variability is generally the largest source of variability. The long whisker on the horizontal variability boxplot for the radionuclides is due to the total uranium measurement. 
Figure 5-1. RSD Distributions for Variance Components Calculated from the Composite-Level Data.
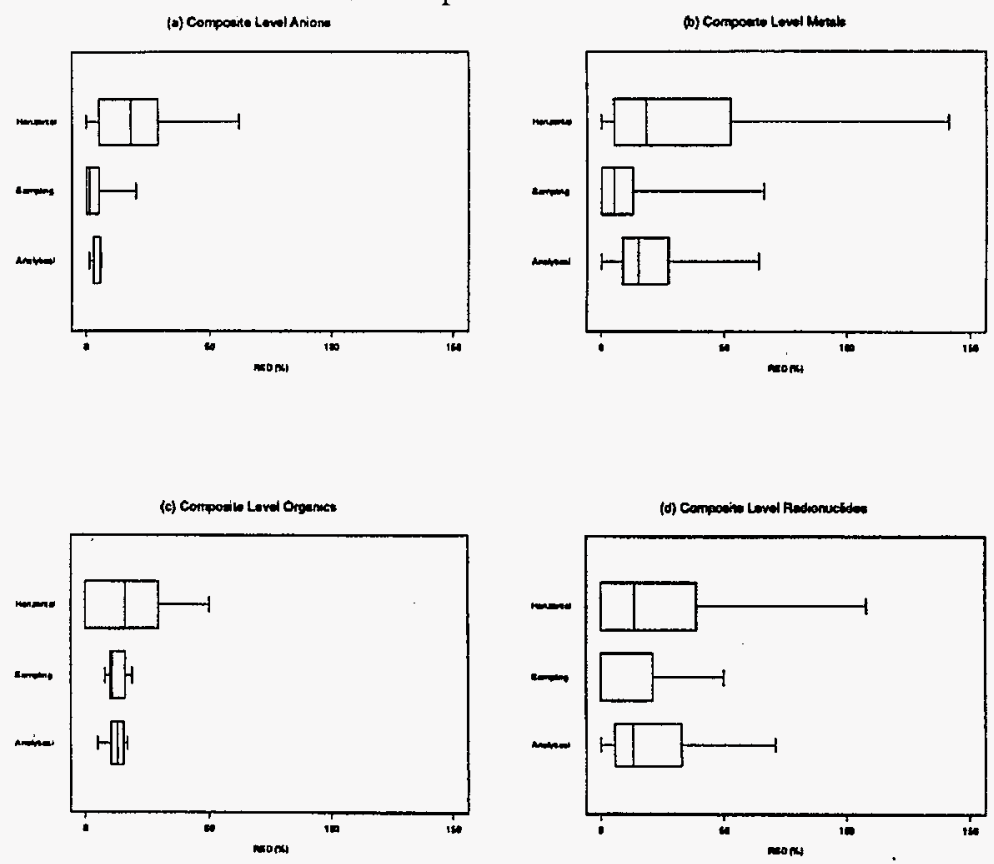

Table 5-3 contains a list of several of the constituents grouped according to the core in which they were found in highest concentration. Constituents were grouped with one core or the other only if the differences between core results were greater than the uncertainty due to sampling and analytical error. For the three constituents measured in highest concentration in B-201 core samples (bismuth, nitrate, and sodium), there were no statistical differences between the cores. However, for a number of the other constituents in large concentration in the core samples (calcium, lanthanum, potassium, iron, phosphorus, silicon), the concentrations found in core 26 were significantly greater than those found in core 27. Constituents were excluded from this analysis if $75 \%$ or more of the sample and duplicate results were below the detection limit (i.e., no ANOVA was run). 
Table 5-3. Analytes Grouped According to Concentration Differences Between Cores.

\begin{tabular}{|c|c|c|}
\hline \multicolumn{3}{|c|}{ Analytes with Higher Concentrations for Core 26} \\
\hline Hexavalent Chromium & Strontium-90 & Cobalt-60 \\
\hline Europium-154 & Aluminum & Barium \\
\hline Calcium* & Chromium & Copper \\
\hline Dysprosium & Lanthanum* & Magnesium \\
\hline Potassium* & Silver & Titanium \\
\hline Vanadium & Iron* & Phosphorus* \\
\hline Silicon* & Chloride & Cyanide \\
\hline Nitrite & Sulfate & Tritium \\
\hline Plutonium-242 & Nickel-59 & Ammonia \\
\hline Pentadecane & Tetradecane & Total carbon \\
\hline \multicolumn{3}{|l|}{ Total inorganic carbon } \\
\hline \multicolumn{3}{|c|}{ Analytes with no Statistical Differences Between Cores } \\
\hline Gross Beta & Americium-241 & Cadmium \\
\hline Cerium & Cobalt & Gadolinium \\
\hline Bismuth* & Manganese* & Molybdenum \\
\hline Nickel & Selenium & Strontium \\
\hline Yttrium & Zinc & Sodium \\
\hline Fluoride & Nitrate* & Zirconium \\
\hline Plutonium-239 & Plutonium-240 & Plutonium-241 \\
\hline Uranium-234 & Uranium-236 & Nickel-63 \\
\hline Dodecane & Tridecane & Total organic carbon \\
\hline \multicolumn{3}{|c|}{ Analytes with Higher Concentrations for Core 27} \\
\hline Gross Alpha & Cesium-137 & Boron \\
\hline Lead & Phosphate & Uranium \\
\hline Uranium-235 & & \\
\hline
\end{tabular}

*Analyte present in concentrations $>5000 \mu \mathrm{g} / \mathrm{g}$.

As the analytical results from B-201 core samples were reviewed, several anomalous results were noted. In many cases, these were excluded from the reported statistical results; while in other cases, they were only identified as "outliers" but used in the analyses.

Table 5-4 shows the sample results that were excluded from the random effects model fits. These results were excluded because of their large disagreements with the other results for a particular constituent. The core 26 and core 27 Laboratory Reports (Shaver 1993) were consulted in order to assign reasons for anomalous results. 
Table 5-4. Composite Values Omitted from Analyses as Suspect.

\begin{tabular}{|ll|cccccc|}
\hline \multicolumn{1}{|c|}{ Analyte } & Method & Core & Composite & Aliquot & Value & $\begin{array}{c}\text { Below } \\
\text { DL }\end{array}$ & Units \\
\hline Bismuth & ICP:W & 26 & 1 & 1 & 49.61 & yes & $\mu \mathrm{g} / \mathrm{g}$ \\
\hline Iron & ICP:W & 26 & 1 & 1 & 16.8 & no & $\mu \mathrm{g} / \mathrm{g}$ \\
\hline Nickel & ICP:W & 26 & 1 & 1 & 14.883 & yes & $\mu \mathrm{g} / \mathrm{g}$ \\
\hline Strontium & ICP:W & 26 & 1 & 1 & 2,481 & yes & $\mu \mathrm{g} / \mathrm{g}$ \\
\hline Arsenic & ICP:F & 26 & 1 & 2 & 636.7 & no & $\mu \mathrm{g} / \mathrm{g}$ \\
\hline Copper & ICP:F & 26 & 1 & 2 & 358.5 & no & $\mu \mathrm{g} / \mathrm{g}$ \\
\hline Iron & ICP:F & 27 & 1 & 2 & 12409 & no & $\mu \mathrm{g} / \mathrm{g}$ \\
\hline Lead & ICP:F & 27 & 1 & 2 & 1959 & no & $\mu \mathrm{g} / \mathrm{g}$ \\
\hline Chloride & IC:W & 26 & 2 & 2 & 2000 & no & $\mu \mathrm{g} / \mathrm{g}$ \\
\hline Fluoride & IC:W & 26 & 2 & 2 & 7200 & no & $\mu \mathrm{g} / \mathrm{g}$ \\
\hline Nitrate & IC:W & 26 & 2 & 2 & 59000 & no & $\mu \mathrm{g} / \mathrm{g}$ \\
\hline Phosphate & IC:W & 26 & 2 & 2 & 1300 & no & $\mu \mathrm{g} / \mathrm{g}$ \\
\hline Cobalt-60 & GEA:F & 26 & 1 & 2 & 0.00859 & no & $\mu \mathrm{Ci} / \mathrm{g}$ \\
\hline Tritium & Liq Scintillation & 26 & 2 & 1 & 0.113 & no & $\mu \mathrm{Ci} / \mathrm{g}$ \\
\hline Uranium-235 & Mass Spectrometry & 26 & 2 & 2 & 0.5825 & no & $\%$ \\
\hline
\end{tabular}

The results reported in Table 5-4 for bismuth, iron, nickel, and strontium (ICP:W) are all from the same aliquot. These four constituents are not expected to be water soluble, and all of the analytical results are close to the detection limits. Because of these conditions, the variability for these analytes is expected to be large. Hence, these results were removed from the random effects model fits.

The arsenic and copper results (ICP:F) reported in Table 5-4 are from the same aliquot. For both constituents, the results from this aliquot are much larger than the other results from the same core (core 26), and these two results were removed from the model fits.

The lead and iron results (ICP:F) reported in Table 5-4 are from the same aliquot. The RPDs for the primary and duplicate results on three of the composites were small for three pairs (e.g., 1\%). The RPD for the other composite (core 26, composite 1) was somewhat larger (19.1\% for lead and $6.6 \%$ for iron). The primary result is closer to the range of results from the other three composites. For this reason, the duplicate results (core 26, composite 1) for iron and lead were dropped from the model fits.

The results reported in the table for chloride, fluoride, nitrate, and phosphate (IC:W) are also from the same aliquot (core 26 , composite 2, aliquot 2). This aliquot result is unusually higher than the other three results from the same core, which are in agreement with one another. The core 26 data report (Shaver 1993) notes that the RPDs are high for 
the duplicate pairs from core 26 . That report attributes the large RPD values to poor sample homogenization. For this reason, these results were removed from the random effects model fits.

The cobalt-60, tritium, and uranium-235 results reported in Table 5-4 were outside the range of other results for the given constituent. The core 26 data report (Shaver 1993) indicates that the process blanks showed significant tritium contamination from previous tritium work. These three results were not used in the random effects model fits.

Figure 5-2 shows a residuals pattern that was noted for several constituents analyzed by the ICP:A method. This plot shows the predicted values from the random effects model fit plotted against the residuals from the fit. If the random effects model (Equation 1) were valid, this plot would show a horizontal band of residuals varying evenly about zero. The four residuals that have the largest absolute values are from core 26 , composite 1 . The core 26 data report (Shaver 1993) notes that these large differences between the sample and duplicate results are due to poor sample homogenization or poor subsampling. These anomalous results were not removed from the statistical analysis, however, because of the limited amount of data available.

Figure 5-2. Residuals Plot for ICP Acid Digestion Aluminum Analysis. Predicted VS Residuals for Aluminum

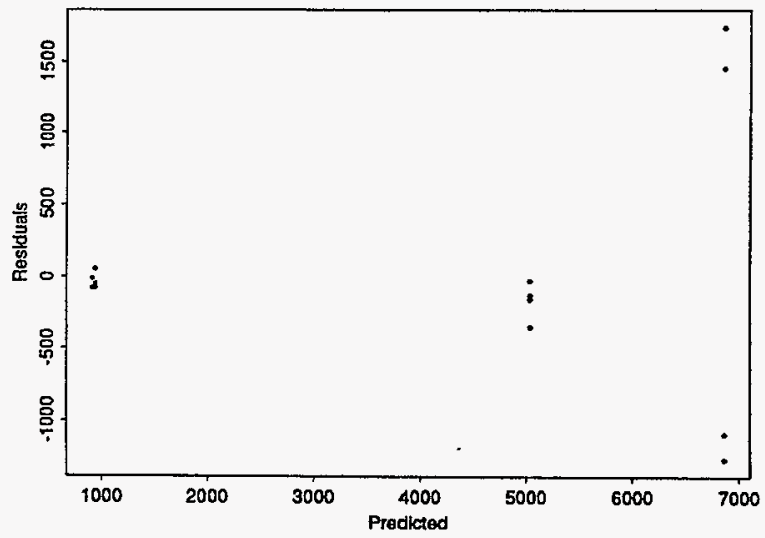

Two different types of ICP fusion analyses were performed on B-201 core samples. The first used sodium peroxide with a zirconium crucible. It was noted that this method created an unknown white precipitate during sample analyses. The second used potassium 
hydroxide with a nickel crucible. The sample results from the sodium peroxide method were excluded from any results presented in this report, except for nickel and potassium. Nickel and potassium results are reported, since the ICP results with potassium hydroxide fusion are invalid for these two constituents.

\subsection{PHYSICAL MEASUREMENTS}

The physical measurements made on the waste are summarized in Table 5-5, which shows the averages of the available measurements for the as-received sample, 1:1 and 3:1 water to sample dilutions. The measurements were made on several segments of core 26 . Since the waste materials in cores 26 and 27 are visually different, lateral heterogeneity is suspected, and the summaries in the table may provide a biased description of the waste's physical properties. A preferable set of measurements would include complete segment-level measurements on both cores, so that both horizontal and vertical variability could be adequately assessed.

Table 5-5. Summary of Core 26 Physical Measurements.

\begin{tabular}{|c|c|c|c|c|}
\hline \multirow{2}{*}{ Analyte } & \multirow{2}{*}{ Units } & \multicolumn{3}{|c|}{ Segments } \\
\hline & & 2 & 5 & 8 \\
\hline \multicolumn{5}{|c|}{ Segment - As Received } \\
\hline Weight $\%$ settled solids & $\%$ & 100 & 100 & 100 \\
\hline Density & $\mathrm{g} / \mathrm{mL}$ & 1.65 & 1.51 & 1.34 \\
\hline Volume \% centrifuged solids & $\%$ & $\overline{98}$ & 98 & 88 \\
\hline Weight $\%$ centrifuged solids & $\%$ & 98 & 98 & 90 \\
\hline Centrifuged Supernate Density & $\mathrm{g} / \mathrm{mL}$ & 1.19 & 1.19 & 1.05 \\
\hline Centrifinged Soltds Density & $\mathrm{g} / \mathrm{mL}$ & 1.66 & 1.52 & 1.37 \\
\hline Shear Strength & dynes $/ \mathrm{cm}^{2}$ & 14100 & 13100 & 12200 \\
\hline \multicolumn{5}{|c|}{ Segment - 1:1 Water to Sample Dilution } \\
\hline Volume \% Settied Solids & $\%$ & 83 & 81 & 92 \\
\hline Density & $\mathrm{g} / \mathrm{mL}$ & 1.33 & 1.17 & 1.13 \\
\hline Volume $\%$ centrifuged solids & $\%$ & 58 & 43 & 42 \\
\hline Weight $\%$ centrifuged solids & $\%$ & 69 & 52 & 49 \\
\hline Centrifuged Supernate Density & $\mathrm{g} / \mathrm{mL}$ & 1.01 & 1.00 & 0.99 \\
\hline Centrifuged Solids Density & $\mathrm{g} / \mathrm{mL}$ & 1.59 & 1.40 & 1.33 \\
\hline \multicolumn{5}{|c|}{ Segment - 3:1 Water to Sample Dilution } \\
\hline Volume \% Settled Solids & $\%$ & 42 & 37 & 57 \\
\hline Density & $\mathrm{g} / \mathrm{mL}$ & 1.10 & 1.05 & 1.05 \\
\hline Volume \% centrifuged solids & $\%$ & 24 & 16 & 21 \\
\hline Weight \% centrifuged solids & $\%$ & 32 & 21 & 25 \\
\hline Centrifuged Supernate Density & $\mathrm{g} / \mathrm{mL}$ & 0.99 & 1.00 & 0.99 \\
\hline Centrifuged Solids Density & $\mathrm{g} / \mathrm{mL}$ & 1.48 & 1.36 & 1.24 \\
\hline
\end{tabular}


The values shown for the as-received segment samples are more appropriate descriptions of the individual segments, rather than representative of the tank as a whole. For example, the segment level densities are much higher than the calculated bulk density of $1.25 \mathrm{~g} / \mathrm{mL}$. The values for the water to sample dilutions represent the potential matrix characteristics if the waste is sluiced.

The RSD reported in Appendix C for these analysis represents spatial RSD, not the uncertainty in the reported measurement. Since measurements were typically taken on several vertical segments within a single core, the spatial variability represented in the RSD is vertical variability.

\subsubsection{Physical and Rheological Properties}

The important physical measurements recorded include density, temperature (in situ), and three different measurements of weight percent solids.

The estimated mean for weight percent solids was $39.3 \%$. This result was based on the analysis of core 26 composite samples only. This value was also used to calculate the estimated percent water $(60.7 \%)$ in the mass and charge balance discussed in Section 7.0 . . Table 5-6 shows the weight percent solids on a segment level basis for both core 26 and 27 . A substantial lateral heterogeneity is exhibited, with core 26 having a much higher water content.

Table 5-6. Weight Percent Solids.

\begin{tabular}{|l|l|l|}
\hline \multicolumn{1}{|c|}{ Segment } & Core 26 & \multicolumn{1}{c|}{ Core 27 } \\
\hline 1 & 51.8 & 33.5 \\
\hline 2 & 53.6 & 28.4 \\
\hline 3 & 29.5 & 96.7 \\
\hline 4 & 29.8 & 42.8 \\
\hline 5 & 31.6 & 46.9 \\
\hline 6 & 33.5 & 66.4 \\
\hline 7 & 28.9 & 82.6 \\
\hline 8 & 28.0 & 68.3 \\
\hline Composite 1 & 39.0 & No Measurement \\
\hline Composite 2 & 39.5 & No Measurement \\
\hline
\end{tabular}

The $\mathrm{pH}$ of the water leaches of the core 26 composite materials (100:1 dilution followed by filtration) and of 10:1 water-to-sample slurries of the core composite materials was measured according to PNL-ALO-225. The average $\mathrm{pH}$ for the water leaches of the 
composites was 8.5 and 8.6 for composites 1 and 2, respectively. The calibration check (made after the measurement of the samples) using a pH 7 buffer was out of control. The measured $\mathrm{pH}$ for this calibration check was 6.5. The $\mathrm{pH}$ of the $10: 1$ slurries was 11.0 for each of the core composite slurries. All calibrations and checks were in control during and after the analysis of the 10:1 slurry sample.

The $\mathrm{pH}$ of the water leaches of the core 27 composite materials (100:1 dilution followed by filtration) was measured in duplicate, according to PNL-ALO-225. The pH for the water leaches of the composites was 8.3 for both composites 1 and 2 .

The penetration resistance for core 26 was measured on each of the extruded segments except segment 1 , which did not have enough solids to make an accurate measurement. The penetration measurement was made on the unhomogenized segment material prior to any further subsampling. These measurements were made after the sample had been sealed in a bottle for approximately six months. The penetration resistance for all segments was less than $3 \mathrm{psi}$; therefore, the sludge is cohesive and will be broken into pieces by the mixer pump instead of being eroded. The penetration resistance is calculated by dividing the measured resistance by 16 , since the 1 -inch shoe was used to measure these minimal resistances.

The penetration resistance for core 27 was measured on each of the extruded segments. This measurement was made on the unhomogenized segment material prior to any further subsampling. These measurements were made after the sample had been sealed in a bottle for approximately one year. The penetration resistance for all segments was greater than $1 \mathrm{psi}$. The penetration resistance is calculated by dividing the measured resistance by 16 , since the 1-inch shoe was used in place of the 1/4-inch shoe. The 1-inch shoe is used to measure small penetration resistances. These low penetration resistances indicate that the sludge is cohesive.

To summarize, the penetrometer readings were all less than 3 psi (the reporting limit), indicating that the waste is not very cohesive. From the rheological measurements, one can draw the conclusion that the waste is not solid and can be pumped. Further rheological analysis regarding the flow behavior of the waste can be found in Appendix E.

\subsubsection{Energetics}

The most notable observation drawn from the thermal analysis is that no exotherms were found. Thermal measurements were made on all eight segments of core 27 , so it can be relatively assured that no exothermic layer exists in this waste. Table 5-7 shows the individual results for each segment in core 27. 
Table 5-7. Core 27 Differential Scanning Calorimetry

Thermal Measurements.

\begin{tabular}{|l|c|c|c|c|c|c|}
\hline \multirow{2}{*}{ Segment } & \multicolumn{3}{|c|}{ Transition 1 } & \multicolumn{3}{c|}{ Transition 2 } \\
\cline { 2 - 7 } & $\begin{array}{c}\text { Enthalpy } \\
(\mathbf{c a l} / \mathbf{g})\end{array}$ & $\begin{array}{c}\text { Onset } \\
\left({ }^{\circ} \mathbf{C}\right)\end{array}$ & $\begin{array}{c}\text { Range } \\
\left({ }^{\circ} \mathbf{C}\right)\end{array}$ & $\begin{array}{c}\text { Enthalpy } \\
(\mathbf{c a l} / \mathbf{g})\end{array}$ & $\begin{array}{c}\text { Onset } \\
\left({ }^{\circ} \mathbf{C}\right)\end{array}$ & $\begin{array}{c}\text { Range } \\
\left({ }^{\circ} \mathbf{C}\right)\end{array}$ \\
\hline 1 & 295 & 64 & $34-140$ & 16 & 143 & $132-217$ \\
\hline 2 & 290 & 73 & $34-141$ & 88 & 158 & $137-340$ \\
\hline 3 & NO & NO & NO & 4 & 123 & $106-153$ \\
\hline 4 & 272 & 64 & $34-125$ & 8 & 134 & $125-191$ \\
\hline 5 & 251 & 66 & $34-139$ & 14 & 146 & $135-235$ \\
\hline 6 & 210 & 62 & $37-183$ & 8 & 153 & $147-210$ \\
\hline 7 & 135 & 46 & $37-146$ & NO & NO & NO \\
\hline 8 & 200 & 51 & $35-158$ & NO & NO & NO \\
\hline
\end{tabular}

NO: Not Observed.

However, the thermal analysis did identify two endotherms in the waste, which together generally absorbed over $800 \mathrm{~J} / \mathrm{g}(200 \mathrm{cal} / \mathrm{g})$. These two endotherms occurred between ambient and $140^{\circ} \mathrm{C}\left(284^{\circ} \mathrm{F}\right)$; and between 140 and $223^{\circ} \mathrm{C}\left(433^{\circ} \mathrm{F}\right)$. These endotherms are believed to be the result of the loss of free and bound water.

\subsubsection{Particle Size Analysis}

The particle size distribution was measured on unhomogenized material from each of the segments of core 26 . The particle size analyzer determines particle sizes in the range of 0.5 to $150 \mu \mathrm{m}$ by measuring the time required for a rapidly moving laser beam to traverse selected particles maintained in a stirred suspension. A glass sphere reference (Duke 147) was measured prior to running the samples, to ensure proper operation of the instrument.

Results from this analysis show that most of the particles in these samples are less than 6 microns in diameter, based on the number density. The volume density data indicates that there is a small percentage of particles of much larger size, but it appears that only a few of the particles exceed 80 microns in diameter. Individual quantitative segment results for core 26 were not found in the data package, however, distribution profiles were presented.

The particle size distribution for core 27 was measured on unhomogenized material from each of the segments. This analysis was performed according to PNL-ALO-530 Rev 0. Results show that most of the particles in these samples are less than 2 microns in diameter, based on the number density. The median particle diameters based on number and volume densities are $0.91 \pm 0.06$ and $22.4 \pm 13.2$ microns, respectively. The volume density data 
indicates that there is a small percentage of particles of much larger size, but it appears that only a few of the particles exceed 100 microns in diameter. Table 5-8 provides the individual segment results for core 27 , and again, distribution profiles can be found in the data package. No discernible trend is observed in the particle size data as a function of depth.

Table 5-8. Particle Size Distribution for Core 27.

\begin{tabular}{|l|cr|rr|}
\hline \multirow{2}{*}{ Segment } & \multicolumn{2}{|c|}{$\begin{array}{c}\text { Particle Size, microns } \\
\text { (by number) }\end{array}$} & \multicolumn{2}{|c|}{$\begin{array}{c}\text { Particle Size, microns } \\
\text { (by volume) }\end{array}$} \\
\cline { 2 - 5 } & \multicolumn{2}{|c|}{ Mean } & Median & \multicolumn{2}{|c|}{ Mean } & Median \\
\hline 1 & 1.13 & 0.88 & 26.37 & 19.98 \\
\hline 2 & 1.31 & 0.91 & 65.55 & 46.62 \\
\hline 3 & 1.48 & 0.92 & 30.47 & 21.63 \\
\hline 4 & 1.07 & 0.84 & 18.02 & 12.08 \\
\hline 5 & 1.16 & 0.87 & 9.42 & 6.46 \\
\hline 6 & 1.56 & 1.03 & 41.79 & 37.49 \\
\hline 7 & 1.24 & 0.93 & 18.65 & 17.45 \\
\hline 8 & 1.10 & 0.86 & 23.24 & 17.65 \\
\hline
\end{tabular}

\subsection{HEAT LOAD ANALYSIS}

The waste in tank B-201 is not very radioactive and consequently generates very little heat through radioactive decay. The most significant radioactive contributors in the waste are strontium-90, cesium-137, and plutonium; contributing 287, 110, and 109 curies, respectively. Table 5-9 summarizes the power produced by the radionuclides in the waste. As one can see, only about 6 watts of heat are produced, not enough energy to power a normal light bulb.

Table 5-9. Radionuclide Inventory and Projected Heat Load.

\begin{tabular}{|r|c|c|c|}
\hline & Total Ci & kW/Ci & $\mathbf{k W}$ \\
\hline Americium-241 & $4.25 \mathrm{e}+00$ & $3.26 \mathrm{e}-05$ & $1.39 \mathrm{e}-04$ \\
\hline Cesium-134 & $3.27 \mathrm{e}-01$ & $1.02 \mathrm{e}-05$ & $3.34 \mathrm{e}-06$ \\
\hline Cesium-137 & $1.10 \mathrm{e}+02$ & $4.72 \mathrm{e}-06$ & $5.19 \mathrm{e}-04$ \\
\hline Cobalt-60 & $2.69 \mathrm{e}-01$ & $1.54 \mathrm{e}-05$ & $4.13 \mathrm{e}-06$ \\
\hline Curium-243/244 & $2.25 \mathrm{e}-01$ & $3.47 \mathrm{e}-05$ & $7.81 \mathrm{e}-06$ \\
\hline Plutonium-238 & $9.47 \mathrm{e}-01$ & $3.33 \mathrm{e}-05$ & $3.16 \mathrm{e}-05$ \\
\hline Plutonium-239/240 & $1.09 \mathrm{e}+02$ & $3.06 \mathrm{e}-05$ & $3.33 \mathrm{e}-03$ \\
\hline Strontium-90 & $2.87 \mathrm{e}+02$ & $6.67 \mathrm{e}-06$ & $1.91 \mathrm{e}-03$ \\
\hline Technetium-99 & $2.66 \mathrm{e}-01$ & $5.00 \mathrm{e}-07$ & $1.33 \mathrm{e}-07$ \\
\hline Total & & & $5.95 \mathrm{e}-03$ \\
\hline
\end{tabular}




\subsection{INTERPRETATION OF ANALYTICAL RESULTS}

This section discusses and interprets the results of the core sample analysis for tank B-201. The first analysis performed was a tank waste profile. This analysis provides information about analyte concentrations as a function of depth. It also provides information about the dominant sources of variability (e.g., spatial or laboratory) in the analytical results. The second analysis is a comparison of the analytical results to the available historical estimates.

\subsection{TANK WASTE PROFILE}

Segment-level measurements can be used to describe the vertical variability of a tank, and to create a tank profile. However development of such a profile is subject to two important limitations. First, the segments allow no finer resolution than 19 in., because they are homogenized before measurement. Secondly, all core segments have not been processed through the same analytical suite in the chemistry lab. In many tanks, sampling plans call for only composite measurements to be taken. The current tank is an example of this guidance. For tank B-201, the only segment-level measurements taken were for the homogenization test.

In this section, the homogenization test data is used to evaluate spatial variability within the tank and to produce a crude tank profile. Homogenization measurements were taken on approximately 50 constituents. These measurements were generally taken on only two segments of the eight that make up a core. One measurement was taken from the middle of the tank (segment 3) and one from the bottom of the tank (segment 6 or 7), so only an incomplete tank profile can be constructed.

Table 6-1 presents a summary of the segment-level measurements available. As one can see from the table, the most complete measurements appear to be the physical measurements. For many physical properties, all 8 segments within a core were measured. However, the measurements were typically made on only one core. 
Table 6-1. Available Segment-Level Measurements.

\begin{tabular}{|c|c|c|c|c|c|c|c|c|}
\hline \multirow[b]{2}{*}{ Core } & \multicolumn{8}{|c|}{ Segments } \\
\hline & 1 & 2 & 3 & 4 & 5 & 6 & 7 & 8 \\
\hline & \multicolumn{8}{|c|}{ Anions } \\
\hline 26 & 0 & 0 & 4 & 0 & 0 & 0 & 4 & 0 \\
\hline \multirow[t]{2}{*}{27} & 0 & 0 & 8 & 0 & 0 & 8 & 0 & 0 \\
\hline & \multicolumn{8}{|c|}{ Metals } \\
\hline 26 & 0 & 0 & 176 & 0 & 0 & 0 & 176 & 0 \\
\hline \multirow[t]{2}{*}{27} & 0 & 0 & 352 & 0 & 0 & 352 & 0 & 0 \\
\hline & \multicolumn{8}{|c|}{ Organics } \\
\hline 26 & 74 & 0 & 74 & 0 & 0 & 0 & 74 & 0 \\
\hline \multirow[t]{2}{*}{27} & 0 & 0 & 0 & 0 & 0 & 0 & 0 & 0 \\
\hline & \multicolumn{8}{|c|}{ Physical } \\
\hline 26 & 8 & 43 & 5 & 5 & 43 & 5 & 5 & 43 \\
\hline \multirow[t]{2}{*}{27} & 27 & 27 & 15 & 27 & 27 & 23 & 19 & 19 \\
\hline & \multicolumn{8}{|c|}{ Radionuclides } \\
\hline 26 & 0 & 0 & 31 & 0 & 0 & 0 & 20 & 0 \\
\hline 27 & 0 & 0 & 44 & 0 & 0 & 40 & 0 & 0 \\
\hline
\end{tabular}

To evaluate this data, a two-way ANOVA model was fit to it. The ANOVA model has the form:

$$
Y_{i j k}=\mu+C_{i}+S_{j}+C S_{i j}+E_{i j k}
$$

where:

$Y_{i j k}=$ The measured value of concentration of a constituent in segment $j$ of core $i$

$\mu=$ The mean concentration of the constituent in the tank

$C_{i}=$ The deviation of core $i$ from the mean (i.e., horizontal variability)

$S_{j}=$ The deviation of segment $j$ from the mean (i:e., vertical variability)

$C S_{i j}=$ Represents general spatial deviations from the mean

$E_{i j k}=$ Represents sampling and analytical deviations. 
The ANOVA algorithm produces variance estimates for each of the deviations listed in the model above:

$$
\begin{aligned}
& \sigma_{C}^{2}=\text { Horizontal variability estimate } \\
& \sigma_{S}^{2}=\text { Vertical variability estimate } \\
& \sigma_{C S}^{2}=\text { General spatial variability estimate } \\
& \sigma_{E}^{2}=\text { Residual variability estimate }
\end{aligned}
$$

as well as estimates for all of the model terms. These variance estimates provide the best summaries of tank spatial homogeneity.

For example, if $\sigma_{C}$ is dominant, then there are large horizontal variations in the waste. If $\sigma_{S}$ is dominant, then the waste has very definite layers. Finally, if $\sigma_{C S}$ is dominant, then the waste is spatially inhomogeneous, but the inhomogeneities are not associated with the vertical or horizontal direction in the tank.

For this particular data set, the segment term was collapsed into three levels: top (segment 1), middle (segments 2 through 5), and bottom (segments 6 through 8).

Appendix $\mathrm{C}$ provides a complete description of the ANOVA results. The first part of Appendix $\mathrm{C}$ lists the variance estimates for all the constituents available and the second part provides tank profile plots.

Table 6-2 summarizes the spatial variabilities found in the tank. Vertical variability is the largest, with general spatial variability $\sigma_{C S}$ a close second. Horizontal variability is the smallest observed spatial variability, although from a qualitative standpoint it appears significant as well (e.g., the visually discernable difference observed between cores 26 and 27).

Table 6-2. Summary of RSD Values.

\begin{tabular}{|l|cccc|}
\hline & \multicolumn{4}{|c|}{ Mean RSD (\%) } \\
& $\sigma_{\mathrm{C}}$ & $\sigma_{\mathrm{S}}$ & $\sigma_{\mathrm{Cs}}$ & $\sigma_{\mathrm{E}}$ \\
\hline Anions & 0 & 13 & 13 & 4 \\
Metals & 14 & 44 & 33 & 14 \\
Organics & & 38 & & 27 \\
Physical & 10 & 17 & 11 & 28 \\
Radionuclides & 18 & 31 & 16 & 31 \\
\hline
\end{tabular}


One can also classify the individual constituents by their variabilities. These results are presented in Table 6-3. From this perspective, vertical variability is largest for 29 constituents, and horizontal for only 3; a further indication that layering is the dominant type of spatial variability in this tank.

Table 6-3. Analytes Classified by Dominant RSD.

\begin{tabular}{|l|ll|}
\hline \multicolumn{1}{|c|}{ Core $\left(\sigma_{S}\right)$} & & Segment $\left(\sigma_{s}\right)$ \\
\hline Temperature & Phosphorus* & Aluminum \\
Europium-155 & Bismuth & Calcium \\
& Chromium & Copper \\
& Iron & Lanthanum \\
& Lead & Magnesium \\
& Nickel & Potassium \\
& Sodium & Strontium \\
& Zinc & Hexamethyldisiloxane \\
& Methoxytrimethylsilane & Toluene \\
& Trimethylsilanol & Centrifuged solids \\
& Density & Critical flow rate-2inch \\
& Critical flow rate-3inch & Critical velocity-2inch \\
& Critical velocity-3inch & Shear strength \\
& Yield point & Americium-241 \\
& Cesium-137 & \\
\hline Core $x$ Segment $\left(\sigma_{C S}\right)$ & & Residuals ( $\left.\sigma_{E}\right)$ \\
\hline Phosphorus* & Manganese & Molybdenum \\
Barium & Silver & Uranium \\
Boron & Hexamethyldisiloxane & Centrifuged supernate density \\
Silicon & Density & Flow behavior index \\
Titanium & Particle size number density mean & Particle size volume density mean \\
Zirconium & Reynolds number-2inch & Reynolds number-3inch \\
& Settled solids & Volume \% centrifuged solids \\
& Weight loss \% & Weight \% centrifuged solids \\
& Weight \% solids & Cesium-134 \\
& Cobalt-60 & \\
\hline
\end{tabular}

*Phosphorus $\sigma_{\mathrm{s}}$ and $\sigma_{\mathrm{CS}}$ are equal.

\subsection{WASTE SUMMARY AND CONDITIONS}

Table 6-4 presents a comparison of historical data to current sampling results. The second and third columns in this table present the best predictions by LANL (Table 2-1) and the 1978 sampling campaign (Table 3-2). The second column presents the most authoritative set of historical estimates and this column should be compared to the fourth column (composite sampling results) to determine how good the LANL historical estimates are. 
Table 6-4. Comparison of Mean Tank Estimates (Historical versus Sampling Results).

\begin{tabular}{|c|c|c|c|c|c|c|}
\hline \multirow[b]{2}{*}{ Constituent } & \multicolumn{2}{|c|}{ Historical } & \multicolumn{2}{|c|}{ Composite Data } & \multicolumn{2}{|c|}{ RPE* } \\
\hline & LANL & 78 Sampling & Est. & \%-RSD & LANL & 78 \\
\hline & \multicolumn{4}{|c|}{$(\mu \mathrm{g} / \mathrm{g})$} & & \\
\hline Aluminum & 0 & 10000 & 3440 & 74 & 100 & 191 \\
\hline Bismuth & 29600 & 38000 & 94100 & 3 & -68.6 & -59.6 \\
\hline Calcium & 0 & NA & 12200 & 58 & -100 & NA \\
\hline Carbonate & 0 & 33000 & NA & NA & NA & NA \\
\hline Chloride & 0 & 100 & 1650 & 7 & -100 & -93.9 \\
\hline Chromium & 1070 & 500 & 3340 & 4 & -68 & -85 \\
\hline Fluoride & 5130 & NA & 5830 & 2 & -12 & NA \\
\hline Hydroxide & 8420 & 6000 & NA & NA & NA & NA \\
\hline Iron & 0 & 18000 & 13400 & 20 & -100 & 34 \\
\hline Lanthanum & 0 & 13000 & 15100 & 10 & -100 & -13.9 \\
\hline Manganese & 5770 & 30 & 19200 & 29 & -69.9 & -99.8 \\
\hline Mercury & 0 & 500 & 0.599 & 46 & -100 & 83400 \\
\hline Nickel & 0 & 1000 & 479 & 3 & -100 & 109 \\
\hline Nitrate & 71300 & 33000 & 49300 & 1 & 44.6 & -33.1 \\
\hline Nitrite & 0 & 40 & 881 & 13 & -100 & -95.5 \\
\hline Oxalate & 16800 & NA & NA & NA & NA & NA \\
\hline Phosphate & 17000 & 11000 & 1210 & 15 & 1310 & 809 \\
\hline Potassium & 9010 & 3000 & 5810 & 13 & 55.1 & -48.4 \\
\hline Silicate & 0 & 3000 & 29700 & 75 & -100 & -89.9 \\
\hline Sodium & 39000 & 28000 & 38200 & 2 & 2.1 & -26.7 \\
\hline Sulfate & 0 & 600 & 348 & 44 & -100 & 72.4 \\
\hline TOC & 4580 & NA & 518 & 10 & 784 & NA \\
\hline Uranium & 0 & 10.5 & 156 & 100 & -100 & -93.3 \\
\hline & & $(\mu \mathrm{Ci} / \mathrm{g})$ & & & & \\
\hline Americium-241 & 0 & 0.005 & 0.031 & 4 & -100 & -83.9 \\
\hline Cesium-137 & 0 & 0.059 & 0.800 & 27 & -100 & -92.6 \\
\hline Europium-155 & 0 & 0.028 & 0.00328 & NA & -100 & 754 \\
\hline Plutonium & 0.964 & $856^{* * *}$ & 1.14 & NA & -15.4 & 74988 \\
\hline Strontium-90 & 0 & 2.7 & 2.09 & 51 & -100 & 29.2 \\
\hline Weight \% solids & 19.9 & 27.8 & 39.3 & $\overline{3}$ & -49.4 & -29.3 \\
\hline
\end{tabular}

*RPE = Relative Percent Error: (Measured - true)/true.

**Measurement not considered credible; likely a transcription error. 
The fourth column contains estimates obtained from the ANOVA fits described in Section 5.0, together with the RSD of the ANOVA estimates. A complete tabulation of our best estimate for each constituent is shown in Table 5-2. The two final columns in Table 6-4 present the relative percent errors for the LANL and 1978 predictions.

Of the 29 constituents listed in Table 6-4, 5 show good agreement, with less than a $\pm 50 \%$ difference between LANL historical results and measured values. Good agreement was obtained for fluoride, nitrate, sodium, plutonium, and weight percent solids.

However, except for those constituents that were missed altogether, the LANL estimates are within a factor of 10 . As a general rule of thumb, one can conclude that when a LANL estimate is present, it is good to within a factor of 10.

It is interesting to note that there are also some significant differences between the results taken in 1978 and the present results. For example, mercury is 3 orders of magnitude larger in the 1978 results than presently. Also, there are very large differences in the radionuclide estimates.

The type of sample that was taken and analyzed is not known; given the poor agreement between the 1978 results and the present results, it is plausible that the 1978 sample might have been of the supernate only.

Table 6-5 provides a means of determining internal consistency for the principal radionuclides. The gross alpha and gross beta measurements (from Table 5-2) are compared to the arithmetic mean of their respective main contributors (sum of alpha emitters = ${ }^{241} \mathrm{Am}+{ }^{239 / 240} \mathrm{Pu}$; sum of beta emitters $\left.=2\left({ }^{90} \mathrm{Sr}\right)+{ }^{137} \mathrm{Cs}\right)$. The comparison shows relatively good agreement in both cases, with RPDs of $12.1 \%$.

Table 6-5. Alpha and Beta Energy Checks.

\begin{tabular}{|l|l|c|}
\hline Calculation & Gross Alpha or Beta & RPD \\
\hline \multicolumn{3}{|c|}{ Total Alpha } \\
\hline${ }^{241} \mathrm{Am}+{ }^{239 / 240} \mathrm{Pu}=1.16 \mu \mathrm{Ci} / \mathrm{g}$ & $1.31 \mu \mathrm{Ci} / \mathrm{g}$ & $12.1 \%$ \\
\hline \multicolumn{3}{|c|}{ Total Beta } \\
\hline $\left.2{ }^{90} \mathrm{Sr}\right)+{ }^{137} \mathrm{Cs}=4.98 \mu \mathrm{Ci} / \mathrm{g}$ & $4.41 \mu \mathrm{Ci} / \mathrm{g}$ & $12.1 \%$ \\
\hline
\end{tabular}


Figure 6-1. RSD Distributions for Variance Components Calculated from the Segment-Level Data.

(a) Segment Level Anions

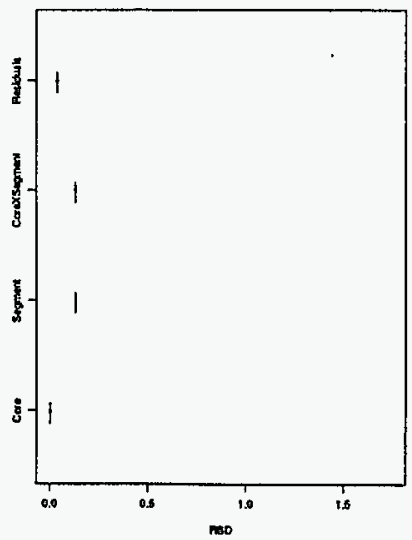

(c) Segment Level Organica

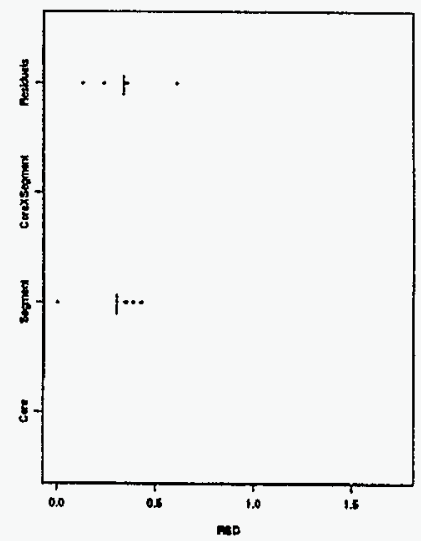

(b) Sogment Lovel Mebats

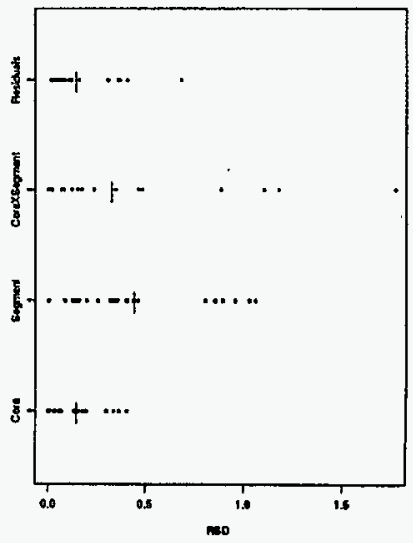

(d) Segment Level Redienuclides

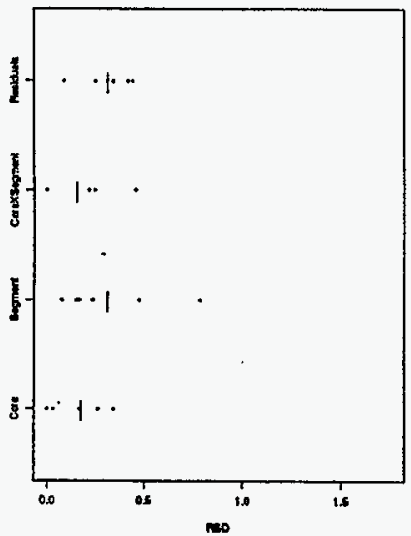


WHC-SD-WM-ER-550 Rev 0

This page intentionally left blank. 


\subsection{STATISTICAL QUALITY ASSURANCE TESTS}

This section contains a summary of the various QA tests and measurements applied to the B-201 analytical results. These tests and measurements include the mass and charge balance, homogenization tests, spike recoveries, and method blanks.

\subsection{MASS AND CHARGE BALANCE}

The principal objective of a mass and charge balance is to determine if the measurements are self-consistent for the composite measurements. The values used in the calculation were obtained from Table 5-2. However, two very important constituents were not measured for this tank: hydroxide and oxygen. To rectify this deficiency, some assumptions had to be made about the missing constituents. Therefore, the mass and charge balances are directed toward filling in information about the missing constituents.

For the mass balance, it is assumed that unmeasured oxygen occurs within five oxyanions, $\mathrm{B}_{4} \mathrm{O}_{7}^{-2}, \mathrm{PO}_{4}^{-3}, \mathrm{SeO}_{3}^{-2}, \mathrm{SiO}_{3}^{-2}$, and $\mathrm{TeO}_{3}^{-2}$. Furthermore assume that all the boron, phosphorus, selenium, silicon, and tellurium measured in the core samples are present in these oxy-anion forms. To determine hydroxide, the charge balance was used: an appropriate amount of hydroxide was added to balance the charges.

Table 7-1 lists the metals used in the mass and charge balances, while Table 7-2 lists the anions. Concentrations of metals and anions are extracted from Table 5-2, which lists the best estimate of the tank contents. These tables also list the RSD associated with each estimate and the postulated charge. The RSDs are used to calculate the uncertainties associated with mass totals.

Table 7-1. Mass/Charge Balance for Metals. (2 pages)

\begin{tabular}{|c|c|c|c|c|c|c|c|}
\hline \multirow[b]{2}{*}{ Metal } & \multicolumn{2}{|c|}{ Mass } & \multirow{2}{*}{$\begin{array}{l}\text { Charge } \\
\mu \mathrm{mol} / \mathrm{g}\end{array}$} & \multirow[b]{2}{*}{ Metal } & \multicolumn{2}{|c|}{ Mass } & \multirow{2}{*}{$\begin{array}{l}\text { Charge } \\
\mu \mathrm{mol} / \mathrm{g}\end{array}$} \\
\hline & $\mu \mathrm{g} / \mathrm{g}$ & RSD & & & $\mu \mathrm{g} / \mathrm{g}$ & RSD & \\
\hline Aluminum & 3440 & 74 & 382.51 & Antimony & 37 & & 0.91 \\
\hline Arsenic & 60 & & 2.38 & Barium & 86 & 38 & 1.26 \\
\hline Beryllium & 4 & & 0.82 & Bismuth & 94100 & 3 & 1350.85 \\
\hline Cadmium & 5 & 6 & 0.09 & Calcium & 12200 & 58 & 608.78 \\
\hline Cerium & 70 & 11 & 1.49 & Chromium & 3340 & 4 & 192.71 \\
\hline Cobalt & 10 & 4 & 0.33 & Copper & 48 & 67 & 1.52 \\
\hline Dysprosium & 29 & 31 & 0.54 & Europium & 7 & & 0.15 \\
\hline Gadolinium & 169 & 15 & 3.22 & Iron & 14400 & 21 & 773.54 \\
\hline Lanthanum & 15100 & 10 & 326.13 & Lead & 1360 & 9 & $\overline{13.13}$ \\
\hline Lithium & 15 & & 2.15 & Magnesium & $\overline{1510}$ & 58 & 124.22 \\
\hline Manganese & 19200 & 29 & 698.97 & Molybdenum & 19 & 4 & 1.19 \\
\hline Neodymium & 22 & & 0.46 & Nickel & 479 & 3 & 16.32 \\
\hline
\end{tabular}


WHC-SD-WM-ER-550 Rev 0

Table 7-1. Mass/Charge Balance for Metals. (2 pages)

\begin{tabular}{|c|c|c|c|c|c|c|c|}
\hline \multirow{2}{*}{ Metal } & \multicolumn{2}{|c|}{ Mass } & \multirow{2}{*}{$\begin{array}{c}\text { Charge } \\
\mu \mathrm{mol} / \mathrm{g}\end{array}$} & \multirow[b]{2}{*}{ Metal } & \multicolumn{2}{|c|}{ Mass } & \multirow{2}{*}{$\begin{array}{l}\text { Charge } \\
\mu \mathrm{mol} / \mathrm{g}\end{array}$} \\
\hline & $\mu g / g$ & RSD & & & $\mu \mathrm{g} / \mathrm{g}$ & RSD & \\
\hline Palladium & 111 & & 2.09 & Potassium & 5810 & 13 & 148.59 \\
\hline Rhodium & 74 & & 2.17 & Ruthenium & 37 & & 1.10 \\
\hline Sodium & 49600 & 23 & 2157.48 & Strontium & 923 & 5 & 21.07 \\
\hline Thallium & 371 & & 1.82 & Thorium-232 & 594 & & 10.24 \\
\hline $\operatorname{Tin}$ & 596 & & 20.09 & Titanium & 285 & 75 & 23.80 \\
\hline Tungsten & 60 & & 1.95 & Uranium & 156 & 100 & 3.93 \\
\hline Vanadium & 16 & 38 & 1.56 & Yttrium & 8 & 18 & 0.28 \\
\hline Zine & 217 & 6 & 6.64 & Zirconium & 11 & 10 & 0.47 \\
\hline
\end{tabular}

Table 7-2. Anions with postulated Oxy-anions.

\begin{tabular}{|c|c|c|c|c|c|c|}
\hline \multirow[b]{2}{*}{ Anion } & \multicolumn{2}{|c|}{ Mass } & \multirow{2}{*}{$\begin{array}{l}\text { Charge } \\
\mu \mathrm{mol} / \mathrm{g}\end{array}$} & \multicolumn{3}{|c|}{ Postulated Oxygen } \\
\hline & $\mu \mathrm{g} / \mathrm{g}$ & RSD & & Anion & $\mu \mathrm{g} / \mathrm{g}$ & RSD \\
\hline Boron & 70 & 35 & 3.26 & $\mathrm{~B}_{4} \mathrm{O}_{7}^{-2}$ & 183 & 35 \\
\hline Chloride & 1650 & 7 & 46.54 & & & \\
\hline Cyanide & 3 & 28 & 0.13 & & & \\
\hline Fluoride & 5830 & 2 & 306.87 & & & \\
\hline Nitrate & 49300 & 1 & 795.16 & & & \\
\hline Nitrite & 881 & 13 & 19.15 & & & \\
\hline Phosphorus & 5450 & 14 & 527.93 & $\mathrm{PO}_{4}^{-3}$ & 11282 & 14 \\
\hline Selenium & 67 & 9 & 3.38 & $\mathrm{SeO}_{3}^{-2}$ & 41 & 9 \\
\hline Silicon & 29700 & 75 & 2466.16 & $\mathrm{SiO}_{3}^{-2}$ & 50787 & 75 \\
\hline Sulfate & 348 & 44 & 10.88 & & & \\
\hline Tellurium & 74 & & 1.16 & $\mathrm{TeO}_{3}^{-2}$ & 28 & \\
\hline
\end{tabular}

Table 7-3 shows the solubility of the phosphorus (as phosphate) by comparing the water-soluble portion to the total phosphate. The phosphate for this waste matrix is estimated to be 7\% soluble (i.e., the majority of the phosphate is in the insoluble form). 
Table 7-3. Phosphate Solubility.

\begin{tabular}{|c|c|}
\hline Calculation & $\begin{array}{c}\text { Phosphate } \\
\text { Solubility }\end{array}$ \\
\hline$\frac{\mathrm{IC}: \mathrm{W} \mathrm{PO}{ }_{4}^{3-} \text { result }}{\mathrm{ICP}: \mathrm{F} \mathrm{P} \text { result as } \mathrm{PO}_{4}^{3-}}=\frac{1210}{16700} \times 100$ & $7.3 \%$ \\
$\frac{\mathrm{ICP}: \mathrm{W} \text { P result }}{\mathrm{ICP}: \mathrm{F} \mathrm{P} \text { result }}=\frac{413}{5450} \times 100$ & $7.6 \%$ \\
\hline
\end{tabular}

In Table 7-2, the "unmeasured oxygen" has also been listed in the last three columns. Table 7-4 summarizes the mass and charge balances. The second and third columns in Table 7-4 list the total masses of metals, anions, and extra oxygen from Tables 7-1 and 7-2, along with the uncertainty associated with each total (expressed as an RSD). Total charges are listed again in the fourth column and from these total charges, the excess negative charge is determined. This excess negative charge has been assigned to hydroxide, and a charge balance determines the mass of hydroxide listed in the table.

Table 7-4. Summary of Mass/Charge Balance.

\begin{tabular}{|c|c|c|c|}
\hline \multirow[b]{2}{*}{ Source } & \multicolumn{2}{|c|}{ Mass } & Charge \\
\hline & $\mu \mathrm{g} / \mathrm{g}$ & RSD & $\mu \mathrm{mol} / \mathrm{g}$ \\
\hline Metal & 224579 & 7 & 6907 \\
\hline Anion & 93373 & 24 & -4219 \\
\hline Oxygen & 62320 & 61 & \\
\hline Hydroxide & 45697 & & -2688 \\
\hline Subtotal & 425969 & & 0 \\
\hline Postulated $\mathrm{H}_{2} \mathrm{O}$ & 572820 & 11 & \\
\hline Measured $\mathrm{H}_{2} \mathrm{O}$ & 607000 & 3 & \\
\hline $\mathrm{RPD}\left(\mathrm{H}_{2} \mathrm{O}\right)$ & $5.8 \%$ & & \\
\hline Estimated Waste Total (Subtotal $+\mathrm{H}_{2} \mathrm{O}$ ) & 1032969 & & \\
\hline RPE (Total Waste) & $3.3 \%$ & & \\
\hline
\end{tabular}

Finally, postulated $\mathrm{H}_{2} \mathrm{O}$ is determined by summing up the masses of metals, anions, extra oxygen, and hydroxide (in parts per million) and subtracting this number from a million. From Table 7-4, the postulated $\mathrm{H}_{2} \mathrm{O}$ in the waste is $57 \%$, and this number has an uncertainty of $11 \%$. The measured percent water is $61 \%$ (determined from the average composite results given in Table 5-6), with an RSD of $3 \%$. The disagreement is $4 \%$, well 
within the estimate uncertainties. As one can see from this mass balance, the assumptions made concerning hydroxide and oxygen seem to fit well with the data for the composite.

\subsection{HOMOGENIZATION TESTS}

Sample homogenization is a very important step in the process of making representative core composite samples. There were two homogenization steps for core samples from B-201. First, the segments from each core were homogenized; and second, homogenized waste from each segment was assembled and homogenized to create core composites. Samples were taken from the top and bottom of two segments per core (segments 3 and 7 from core 26 and segments 3 and 6 from core 27), and from the top and bottom of each core composite. Analytical results from these samples were used to determine if the sample homogenization was adequate. The segment level samples were prepared by acid digestion and chemically analyzed using ICP and GEA. The composite level samples were prepared using $\mathrm{KOH} / \mathrm{Ni}$ fusion and chemically analyzed using ICP and GEA.

The analytical results from the top and bottom segment and composite samples (homogenization samples) were fit to the following nested random effects model:

$$
Y_{i j k}=\mu+C_{i}+S_{i j}+H_{i j k}+E_{i k k}
$$

where:

$X_{i j k}=$ The measured value of concentration of a constituent in segment $j$ of core $i$

$\mu=$ The mean concentration of the constituent

$C_{i}=$ The core sampled

$S_{i j}=$ The segment or composite from a core

$H_{i j k}=$ The location on the composite or segment (homogenization effect)

$E_{i j k}=$ The analytical error.

The objective of the homogenization test is to determine if the variability in the results between sampling locations is greater than zero. This objective can be met using the results from an ANOVA on the random effects model.

The results from an ANOVA are presented in Table 7-5. The homogenization RSD (estimated variability between locations relative to the mean) is given, along with the p-value from the homogenization tests. Analytes with more than $75 \%$ of the analytical results below the detection limits were excluded from this analysis. 
Table 7-5. Homogenization Test Results.

\begin{tabular}{|c|c|c|c|c|c|c|c|c|c|}
\hline \multicolumn{10}{|c|}{ Composite Level Homogenization Tests (KOH/Ni Fusion ICP and GEA) } \\
\hline \multirow[b]{2}{*}{ Analyte } & \multicolumn{2}{|c|}{ Homogenization } & \multirow[b]{2}{*}{$<D L$} & \multirow[b]{2}{*}{ Obs } & \multirow[b]{2}{*}{ Analyte } & \multicolumn{2}{|c|}{ Homogenization } & \multirow[b]{2}{*}{$<D L$} & \multirow[b]{2}{*}{ Obs } \\
\hline & $\operatorname{RSD}(\%)$ & p-ralue & & & & $\mathbf{R S D}(\%)$ & p-ralue & & \\
\hline Aluminum & 14 & 0.001 & 0 & $\overline{16}$ & Barium & 12 & 0.203 & 0 & 16 \\
\hline Bismuth & 0 & 0.875 & 0 & 16 & Calcium & 12 & 0.000 & 0 & 16 \\
\hline Chromium & 0 & 0.799 & 0 & 16 & Cobalt & 0 & 0.474 & 10 & 16 \\
\hline Copper & 32 & 0.201 & 0 & 16 & Iron & 9 & 0.030 & 0 & 16 \\
\hline Lanthanum & 0 & 0.954 & 0 & 16 & Lead & 0 & 0.818 & 0 & 16 \\
\hline Magnesium & 11 & 0.005 & 0 & 16 & Manganese & 0 & 0.945 & 0 & 16 \\
\hline Phosphorus & 0 & 0.708 & 0 & 16 & Silicon & 13 & 0.035 & 0 & 16 \\
\hline Sodium & 3 & 0.344 & 0 & 16 & Strontium & 0 & 0.925 & 0 & 16 \\
\hline Titanium & 14 & 0.000 & 0 & 16 & Vanadium & 4 & 0.472 & 12 & 16 \\
\hline Zinc & 0 & 0.676 & 0 & 16 & Zirconium & 5 & 0.472 & 12 & 16 \\
\hline Americium-241 & 28 & 0.029 & 0 & 16 & Cesium-134 & NA & NA & 0 & 2 \\
\hline Cesium-137 & 93 & 0.181 & 0 & 16 & Cobalt -60 & 0 & 0.651 & 4 & 12 \\
\hline Europium-154 & 0 & 0.787 & 0 & 12 & Europium-155 & NA & NA & 0 & 8 \\
\hline Potassium- 40 & $\mathrm{NA}$ & NA & 2 & 8 & & & & & \\
\hline \multicolumn{10}{|c|}{ Segment Level Homogenization Tests (Acid Digestion ICP and GEA) } \\
\hline & \multicolumn{2}{|c|}{ Homogenization } & \multirow[b]{2}{*}{$<D L$} & \multirow[b]{2}{*}{ Obs } & \multirow[b]{2}{*}{ Analyte } & \multicolumn{2}{|c|}{ Homogenization } & \multirow[b]{2}{*}{$<D L$} & \multirow[b]{2}{*}{ Obs } \\
\hline Analyte & RSD(\%) & p-value & & & & $\operatorname{RSD}(\%)$ & p-value & & \\
\hline Aluminum & 3 & 0.001 & 0 & 24 & Barium & 3 & 0.154 & 0 & 24 \\
\hline Bismuth & 8 & 0.003 & 0 & 24 & Boron & 0 & 0.909 & 0 & 24 \\
\hline Calcium & 2 & 0.217 & 0 & 24 & Chromium & 5 & 0.015 & 0 & 24 \\
\hline Copper & 7 & 0.006 & 7 & 24 & Iron & 3 & 0.072 & 0 & 24 \\
\hline Lanthanum & 7 & 0.002 & 0 & 24 & Lead & 4 & 0.151 & 4 & 24 \\
\hline Magnesium & 0 & 0.775 & 0 & 24 & Manganese & 0 & 0.997 & 0 & 24 \\
\hline Phosphorus & 4 & 0.029 & 0 & 24 & Nickel & 5 & 0.011 & 0 & 24 \\
\hline Potassium & 1 & 0.305 & 3 & 24 & Silicon & 0 & 0.428 & 0 & 24 \\
\hline Sodium & 6 & 0.003 & 0 & 24 & Strontium & 7 & 0.003 & 0 & 24 \\
\hline Titanium & 1 & 0.177 & 1 & 24 & Zine & 0 & 0.939 & 7 & 24 \\
\hline Americium-241 & 6 & 0.070 & 0 & 24 & Cesium-134 & 14 & 0.217 & 7 & 15 \\
\hline Cesium-137 & 29 & 0.486 & 0 & 24 & Cobalt -60 & 0 & 0.790 & 6 & 18 \\
\hline Europium-154 & 26 & 0.094 & 7 & 15 & Europium-155 & 8 & 0.369 & 8 & 12 \\
\hline
\end{tabular}

(p-value $<.05$ indicates a significant RSD).

The homogenization tests on the composite level show that for $83 \%$ of the analytes tested, the variability due to homogenization cannot be distinguished from zero ( $99 \%$ significance level). In other words, the homogenization on the composite level is sufficient for $83 \%$ of the analytes tested. However, the core 26 report (Shaver 1993) indicates that many of the sample and duplicate RPDs are large (i.e., 10 to $30 \%$, versus less than $10 \%$ at the segment level). These large RPDs may reduce the validity of the statement made above. 
The homogenization tests on the segment level show that for $77 \%$ of the analytes tested, the variability due to homogenization cannot be distinguished from zero (99\% significance level). For the other $23 \%$ of the analytes, the homogenization RSDs are relatively small (i.e., less than $10 \%$ ). Even though variability due to homogenization is tested to be greater than zero (due to small analytical error), the variability is small enough to consider the homogenization adequate. The core 26 report (Shaver 1993) indicates again that there were large RPDs for several duplicate analyses. Due to the generally small homogenization RSDs, the large analytical error from these large RPDs does not affect conclusions about the homogenization as much as for the composites. In general, the homogenization on the segment level is considered adequate.

\subsection{EVALUATION OF SPIKES AND BLANKS}

Of the 7,598 observations in the B-201 data base, 2,480 are some form of spike or blank measurement run for QA purposes. In this section, we present a brief overview of these measurements. More detailed evaluations of the spikes and blanks can be found in the core reports.

Evaluation of blanks uncovered an important problem with the ICP fusion measurements on core 26 . The blanks for many of the constituents were sometimes $50 \%$ of the measured value. When the blank results were compared to homogenization measurements on the same sample, it became apparent that the contamination being measured by the blanks was real.

Table 7-6 illustrates the problem. If one compares the homogenization measurements to the standard measurements, the results differ by approximately $58 \%$, much more than the sample and duplicate replicates differ (about $5 \%$ ). However, if one subtracts the blanks first, then the agreement becomes dramatically better (11\%).

Table 7-6. Illustration of Contamination Problem for ICP fusion on Core 26.

\begin{tabular}{|l|r|r|r|r|r|r|r|r|}
\hline \multirow{2}{*}{ Constituent } & \multicolumn{4}{|c|}{ Standard Measure } & \multicolumn{3}{c|}{ Homogenization Measure } & \multicolumn{2}{c|}{ Adjusted Measure } \\
& \multicolumn{1}{|c|}{ Samp. } & \multicolumn{1}{c|}{ Dup. } & \multicolumn{1}{c|}{ Blank } & \multicolumn{1}{c|}{ Samp. } & \multicolumn{1}{c|}{ Dup. } & \multicolumn{1}{c|}{ Blank } & \multicolumn{1}{c|}{ Samp. } & \multicolumn{1}{c|}{ Dup. } \\
\hline $\mathrm{Al}$ & 12324 & 13071 & 6115 & 6940 & 7183 & $<\mathrm{DL}$ & 6209 & 6956 \\
\hline $\mathrm{B}$ & 14946 & 16567 & 15342 & <DL & $<\mathrm{DL}$ & $<\mathrm{DL}$ & $<\mathrm{DL}$ & $<\mathrm{DL}$ \\
\hline $\mathrm{Ca}$ & 20207 & 20674 & 4065 & 19055 & 19537 & $<\mathrm{DL}$ & 16142 & 16609 \\
\hline $\mathrm{Na}$ & 64039 & 68510 & 26897 & 41843 & 43726 & $<\mathrm{DL}$ & 37142 & 41613 \\
\hline $\mathrm{Si}$ & 55255 & 59723 & 24351 & 29634 & 30620 & $<\mathrm{DL}$ & 30904 & 35372 \\
\hline
\end{tabular}

Extracted from Tables 2-1a and 2-1c of core reports (Shaver 1993).

Values in table are for composite 1. 
It is obvious that these measurements must be corrected for contamination. Blanks were therefore subtracted from the ICP fusion measurements. There are indications that other corrections might be in order. However, it has been the policy to make no such corrections for other tanks at Hanford, so corrections were limited to the ICP fusions for $\operatorname{tank}$ B-201.

\subsubsection{Blank Measurements}

Approximately 1,400 blank measurements were made on tank B-201 samples, the majority for the ICP analytic methods. Most blanks were below the detection limit (83\%). Table 7-7 summarizes the blank measurements taken on tank B-201. The last column in this table shows what percentage of the actual measurement is represented by those blanks that were not below the detection limit. One would like the blanks to be a small percentage of the actual measurement, and Figure 7-1 graphically illustrates the sizes of the blank measurements above the detection limit. From this figure, it is obvious that some blanks are quite large.

Table 7-7. Summary of Blank Measurements.

\begin{tabular}{|l|r|r|r|}
\hline Analyte: Sample Preparation & $\begin{array}{c}\text { \# Blanks } \\
\langle\boldsymbol{D} L\end{array}$ & $\begin{array}{c}\text { \# Blanks } \\
\boldsymbol{T} \boldsymbol{D} \boldsymbol{L}\end{array}$ & $\begin{array}{c}\text { Median Blank } \\
\text { Observed }\end{array}$ \\
\hline AA (As):A & 1 & 1 & 57 \\
\hline Ext Organic Halides & 1 & 2 & 43 \\
\hline Alpha Radchem:F & 3 & 11 & 1 \\
\hline Beta Radchem:F & 4 & 6 & 17 \\
\hline GEA & 3 & 2 & 10 \\
\hline GEA:A & 10 & 7 & 14 \\
\hline GEA:F & 12 & 13 & 25 \\
\hline GEA:W & 0 & 3 & 3 \\
\hline ICP:A & 405 & 101 & 2 \\
\hline ICP:F & 216 & 52 & 24 \\
\hline ICP:W & 176 & 8 & 3 \\
\hline IC:W & 15 & 6 & 13 \\
\hline Liq Scintillation:W & 0 & 3 & 35 \\
\hline Liq Scintillation:A & 6 & 2 & 18 \\
\hline ISE:W & 0 & 3 & 99 \\
\hline SVOA & 195 & 1 & 15 \\
\hline VOA & 93 & 9 & 0 \\
\hline Persulfate Oxidation:W & 2 & 7 & 12 \\
\hline Beta Radchem:W & 0 & 2 & 19 \\
\hline
\end{tabular}


Figure 7-1. Boxplots of Blank/Actual for Each Analytical Method ( $<D L$ Measurements Excluded).

AA (AB):A
Exi Organle Halides
Alpha Radchem:F
Beta Radchem:F
GEA
GEA:A
GEA:F
GEA:W
ICP:A
ICP:F
ICP:W
IC:W
Liq Scintilation:W
Liq Sctrillation:A
ISE:W
SVOA
VOA
Petsuffate Oxidation:W
Beta Radchem:W

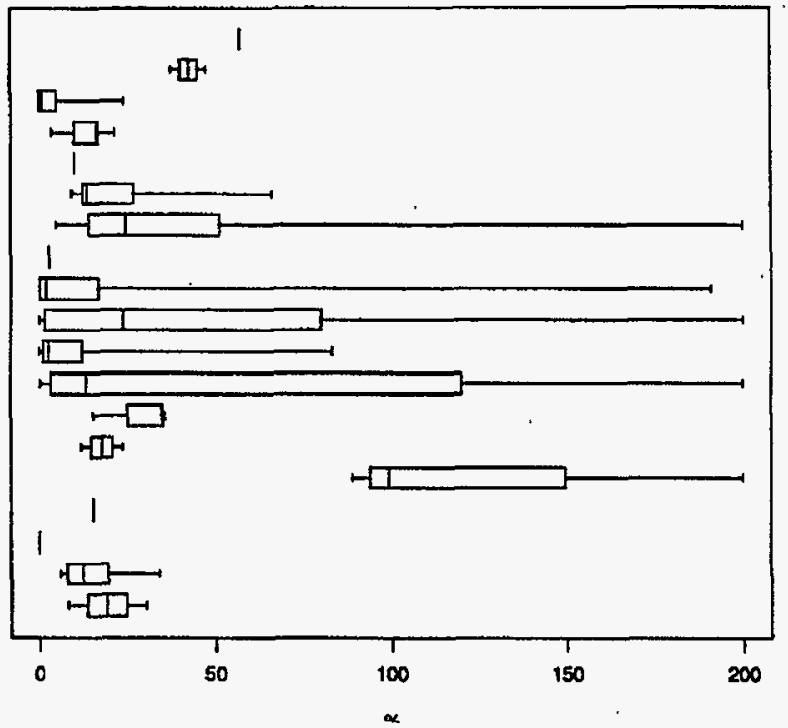

\subsubsection{Spike Measurements}

Figure 7-2 shows the percent recovery distributions for spike measurements of each analysis type. The percent recovery is targeted to be between $75 \%$ and $125 \%$, and this is generally the case for alpha and beta radiation chemistry and ion chromatography. However, as one can see from the box-plots, several analytical methods show much more variability than this. None of the measurements were corrected for recovery problems. 
Figure 7-2. Boxplots \% Recovery Calculated from Spike Measurements.

AA (As):A

AA (SD)'A

AM (Se):A

CVAAIA

Ext Organic Halides

Alpha Radchem:F

Beta Padchem:F

KP:A

K:W

Laser Flurorimetry:F

Lla Seintillation:W

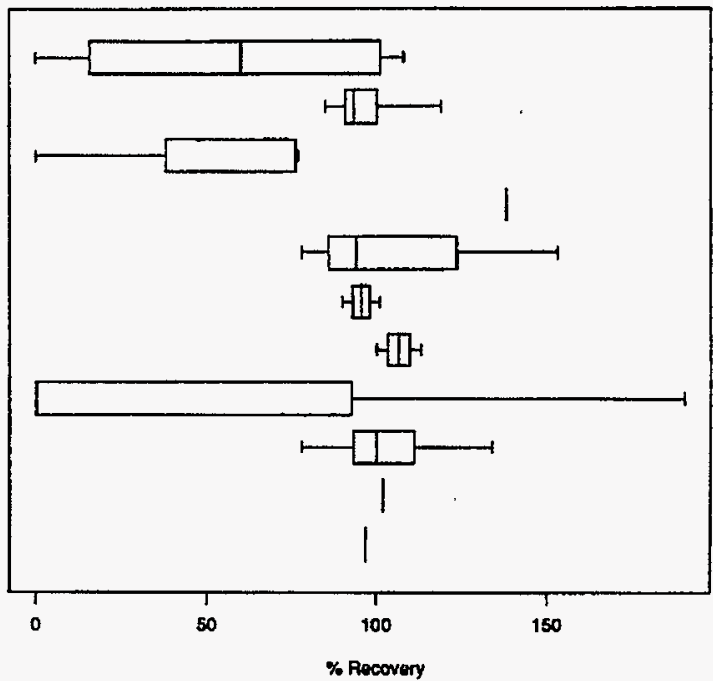

Table 7-8 summarizes the number of spike measurements that were outside the $75 \%$ to $125 \%$ recovery goals. As one can see, ICP acid stands out, with 152 measurements outside the desired range. Most recovery percents on ICP acid are quite small, about $4 \%$. 
Table 7-8. Summary of Recoveries Calculated from Spike Measurements.

\begin{tabular}{|l|c|c|}
\hline \multicolumn{1}{|c|}{$\begin{array}{c}\text { Analyte: } \\
\text { Preparation Method }\end{array}$} & $\begin{array}{c}\text { Outside } \\
\text { Range* }\end{array}$ & $\begin{array}{c}\text { Within } \\
\text { Range* }\end{array}$ \\
\hline AA (As):A & 2 & 2 \\
\hline AA (Sb):A & 0 & 4 \\
\hline AA (Se):A & 1 & 2 \\
\hline CVAA:A & 1 & 0 \\
\hline Ext Organic Halides & 1 & 2 \\
\hline Alpha Radchem:F & 0 & 4 \\
\hline Beta Radchem:F & 0 & 2 \\
\hline ICP:A & 152 & 78 \\
\hline IC:W & 3 & 27 \\
\hline Laser Fluorimetry:F & 0 & 1 \\
\hline Liq Scintillation:W & 0 & 1 \\
\hline
\end{tabular}

$*$ Range $=75 \%$ to $125 \%$.

\subsubsection{Quality Assurance Flags}

Hanford Analytical Services (HAS) reviewed all data and assigned QA flags to the results. Of the 7,598 measurements in the data set, HAS classified $920(12 \%)$ as unusable and another $403(55 \%)$ as suspect (i.e., suspect results are considered to be rough estimates only). In order to perform the analysis presented in this report, all data was used and none of the HAS-flagged data was deleted. 


\subsection{CONCLUSIONS AND RECOMMENDATIONS}

The process waste history for tank B-201 is uncomplicated. Over its years of service, tank B-201 received most of its contents as discharges from the 224 Concentration Building, which had low enough fission product activity $(<0.001 \%$ of that in the starting metal) to permit ground disposal after the waste was directed through a settling tank. The solid portion of the waste (106,000 liters [28,000 gallons]) is a dark brown to black sludge. There are approximately 3,800 liters (1,004 gallons) of supernate above the sludge.

Excluding water, the analytes found in highest concentration $\left(>10^{4} \mathrm{ppm}\right)$ in B-201 core samples are bismuth, nitrate, sodium, silicon, manganese, lanthanum, iron, and calcium. The historical tank concentration estimates for B-201 were obtained from LANL, and supplemented by analytical results from a sampling event in 1978. Presently, the most significant difficulty in using the LANL estimates involves making comparisons with constituents that are not predicted in the waste, but which are actually present. Several constituents (such as aluminum) were predicted to be absent by LANL, but were actually found in significant concentration in the tank samples. Furthermore, there is no quantification of uncertainty with the LANL estimates at this time.

On the other hand, those constituents that LANL did predict were generally within one order of magnitude of the estimates obtained from the composite samples for B-201, making this an acceptable first estimate in the absence of any characterization information.

The ANOVA analysis of the core segment data showed that the vertical spatial variability is generally larger than horizontal variability, and laboratory-related variability is generally the smallest. However, analytical error is a significant contributor to the uncertainty about the mean for those analytes that are close to the detection limits.

From the composite data, the uncertainties in the best estimates are generally dominated by horizontal spatial variability. This has consequences for tank sampling: If more accurate estimates of the tank contents are required, then more core samples must be taken, through different risers. (Improvements in analytic procedures or in sampling methodology would not be adequate).

The QA tests show mixed results as to the usability of the analytical data from B-201 core samples. The mass/charge balance shows relatively good agreement between postulated and measured results. The homogenization test indicates that the waste samples from B-201 were generally mixed sufficiently to produce representative results. The analysis of spikes and blanks, however, reveals some problems with the data. Many of the spike recoveries (\%) for ICP analyses are outside the acceptable range of $\pm 25 \%$. It was also noted in Section 7.3.1 that many blank corrections had to be made for analyses performed by ICP fusion. HAS reviewed the B-201 core reports and flagged $12 \%$ of the data as unusable and $55 \%$ more as suspect.

B-201 is not on any of the watch lists (e.g., ferrocyanide or flammable gas), and therefore has no safety issues that need to be addressed. 
WHC-SD-WM-ER-550 Rev 0

This page intentionally left blank. 


\subsection{REFERENCES}

Anderson J. D., 1990, A History of the 200 Area Tank Farms, WHC-MR-0132, Westinghouse Hanford Company, Richland Washington.

Brevick C. H., 1994, Historical Tank Content Estimate for the Northeast Quadrant of the Hanford 200 East Areas, WHC-SD-WM-ER-349, Rev. 0, Westinghouse Hanford Company, Richland, Washington.

De Lorenzo D. S., J. H. Rutherford, D. J. Smith, D. B. Hiller, K. W. Johnson, and B. C. Simpson, 1994, Tank Characterization Reference Guide, WHC-SD-WM-TI-648, Rev. 0, Westinghouse Hanford Company, Richland, Washington.

DOE, 1994, Hanford Environmental Information System (HEIS), Tank Characterization Data (TCD) Subject Area, DOE/RL-93-24-8, Volume 8, U.S. Department of Energy, Richland Operations Office, Richland, Washington.

Ecology, EPA, and DOE, 1993, Hanford Federal Facility Agreement and Consent Order, as amended, Washington State Department of Ecology, U.S. Environmental Protection Agency, and the U.S. Department of Energy, Olympia, Washington.

HW-9191 DEL, 1948, Hanford Works Monthly Report, February 1948.

HW-9595 DEL, 1948, Hanford Works Monthly Report, March 1948.

Horton J. E., 1978, "Characterization of 200 Series Tanks," Internal Memo 60120-78-131, Rockwell Hanford Operations, Richland, Washington.

Jungfleisch F. M., and B. C. Simpson, 1993, A Preliminary Estimation of the Waste Inventories in Hanford Tanks Through 1980, WHC-SD-WM-TI-057, Rev. 0-A, Westinghouse Hanford Company, Richland, Washington.

PNL-ALO-225, 1990, Measurement of pH in Aqueous Solutions, Pacific Northwest National Laboratory Technical Procedure, Pacific Northwest National Laboratory, Richland, Washington.

PNL-ALO-530, Rev. 0, 1989, Particle Size Distribution By Laser Scanning (Time of Transition), Pacific Northwest National Laboratory Technical Procedure, Pacific Northwest National Laboratory, Richland, Washington.

Shaver R. L., 1993, PNL 325 Laboratories Single-Shell Tank Waste Characterization, Tank B-201 Cores 26 and 27, WHC-SD-WM-DP-037, Westinghouse Hanford Company, Richland, Washington. 
WHC-SD-WM-ER-550 Rev 0

This page intentionally left blank. 


\subsection{BIBLIOGRAPHY}

Babad H., 1994, Tank Safery Screening Data Quality Objective, WHC-SD-WM-SP-004, Westinghouse Hanford Company, Richland, Washington.

Bell K. E., 1993, Tank Waste Remediation System Tank Waste Characterization Plan, WHC-SD-WM-PLN-047, Rev. 1, Westinghouse Hanford Company, Richland, Washington.

Hanlon B. M., 1992, Tank Farm Surveillance and Waste Status Summary Report for September 1992, WHC-EP-0182-39, Westinghouse Hanford Company, Richland, Washington.

Hanlon B. M., 1993, Tank Farm Surveillance and Waste Status Summary Report for November 1993, WHC-EP-0182-68, Westinghouse Hanford Company, Richland, Washington.

Huckaby J. L., 1992, Characterization of Vapors in Single-Shell Tanks Scheduled for Rotary-Mode Sampling, WHC-SD-WM-TI-536, Westinghouse Hanford Company, Richland, Washington.

Winters W. I., L. Jensen, L. M. Sasaki, R. L. Weiss, J. F. Keller, A. J. Schmidt, and M. G. Woodruff, 1990, Waste Characterization Plan for Hanford Site Single-Shell Tanks, WHC-EP-0210, Rev. 1, Westinghouse Hanford Company, Richland, Washington. 
This page intentionally left blank. 
APPENDLX A

TANK ENGINEERING DATA AND WASTE SUMMARY

A-1 
This page intentionally left blank. 


\section{APPENDIX A}

\section{TANK ENGINEERING DATA AND WASTE SUMMARY}

Figure A-1. Top View of Tank B-201.

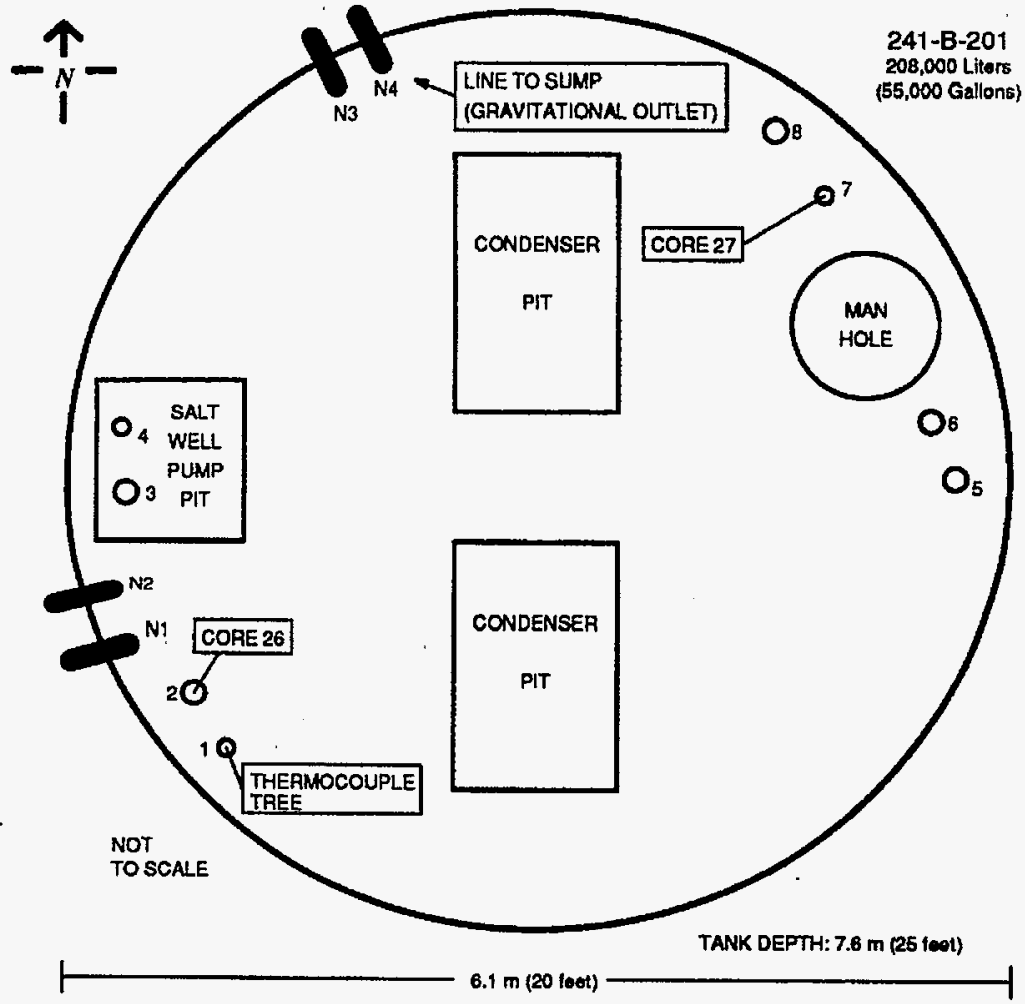


Table A-1. Engineering Data Summary of Tank B-201.

\begin{tabular}{|c|c|c|c|}
\hline \multicolumn{2}{|c|}{ Tank Engineering Description } & \multicolumn{2}{|c|}{ Tank Status as of 1994} \\
\hline Type: & Single-shell tank & Watch List: & None \\
\hline Construction: & $1943-1944$ & Interim Stabilized: & 1981 \\
\hline In-Service: & 1947 & Interim Isolated: & 1981 \\
\hline Out of Service: & 1975 & Contents: & 224 waste* \\
\hline Diameter: & $6.1 \mathrm{~m}(20 \mathrm{ft})$ & Tank Integrity: & Assumed leaker $(1980)(4,500 \mathrm{~L})$ \\
\hline Depth: & $7.6 \mathrm{~m}(25 \mathrm{ft})$ & & \\
\hline Nominal Capacity: & $208,000 \mathrm{~L}(55,000 \mathrm{gal})$ & & \\
\hline Bottom Shape: & Dished & & \\
\hline Hanford Coordinates: & N45537.5, W52727.5 & & \\
\hline Ventilation: & Passive & & \\
\hline
\end{tabular}

*Non-complexed waste.

Table A-2. Inventory Summary for Tank B-201.

\begin{tabular}{|c|c|c|c|}
\hline \multicolumn{4}{|c|}{ Physical Properties of Waste } \\
\hline Total Waste: & $110,000 \mathrm{I}(29000 \mathrm{gal})$ & Supernate Volume: & $3,800 \mathrm{~L}(1,000 \mathrm{gal})$ \\
\hline Drainable Inter. Liquid: & $11,400 \mathrm{~L}(3,000 \mathrm{gal})$ & Density: & $1.25 \mathrm{~g} / \mathrm{mL}$ \\
\hline $\mathrm{H}_{2} \mathrm{O}$ Average: & $60.6 \%$ & Temperature Average: & $17^{\circ} \mathrm{C}\left(63^{\circ} \mathrm{F}\right)$ \\
\hline pH: & 8.53 & Maximum Exotherm: & No Exotherms \\
\hline Heat Load: & $0.006 \mathrm{~kW}$ & & \\
\hline \multicolumn{4}{|c|}{ Chemical Properties of Waste } \\
\hline Bismuth: & $13,000 \mathrm{~kg}(9.45 \mathrm{wt} \%)$ & Calcium: & $1,670 \mathrm{~kg}(1.22 \mathrm{wt} \%)$ \\
\hline Nitrate: & $6,770 \mathrm{~kg}(4.93 \mathrm{wt} \%)$ & Fluoride: & $800 \mathrm{~kg}(0.58 \mathrm{wt} \%)$ \\
\hline Sodium: & $5,240 \mathrm{~kg}(3.82 \mathrm{wt} \%)$ & Potassium: & $797 \mathrm{~kg}(0.58 \mathrm{wt} \%)$ \\
\hline Silicon: & $2,770 \mathrm{~kg}(2.02 \mathrm{wt} \%)$ & Phosphorus: & $748 \mathrm{~kg}(0.55 \mathrm{wt} \%)$ \\
\hline Manganese: & $2,630 \mathrm{~kg}(1.92 \mathrm{wt} \%)$ & TOC: & $71 \mathrm{~kg}(5.18 \mathrm{e}-02 \mathrm{wt} \%)$ \\
\hline Lanthanum: & $2,070 \mathrm{~kg}(1.51 \mathrm{wt} \%)$ & TIC: & $287 \mathrm{~kg}(0.21 \mathrm{wt} \%)$ \\
\hline Iron: & $1,840 \mathrm{~kg}(1.34 \mathrm{wt} \%)$ & & \\
\hline \multicolumn{4}{|c|}{ Radionuclides in the Waste } \\
\hline Total Plutonium: & $156 \mathrm{Ci}$ & Strontium-90: & $287 \mathrm{Ci}$ \\
\hline Cesium-137: & $110 \mathrm{Ci}$ & Total Uranium & $21.4 \mathrm{~kg}(1.56 \mathrm{e}-04 \mathrm{wt} \%)$ \\
\hline
\end{tabular}


APPENDIX B

COMPOSITE ESTIMATES AND VARIABILITY SUMMARY 
WHC-SD-WM-ER-550 Rev 0

This page intentionally left blank. 


\section{APPENDIX B}

\section{COMPOSITE ESTIMATES AND VARIABILITY SUMMARY}

This section contains ANOVA table results for the composite data (including the drainable liquid). The most important value in this table is the average concentration estimate, $\hat{\mu}$, but the table also presents variance component estimates. The model used to produce these results is:

$$
Y_{i j k}=\mu+C_{i}+S_{i j}+E_{i j k}
$$

where:

$Y_{i j k}=$ The measured value of concentration of a constituent in replicate $j$ of core $i$

$\mu=$ The mean concentration of the constituent in the tank

$C_{i}=$ The deviation of concentration in core $i$ from the mean value

$S_{i j}=$ The deviation of concentration in core replicates. (Two replicates were processed on each composite.)

$E_{i j k}=$ The analytical (lab) error in the measurements.

All RSDs presented in this appendix are in percent. The RSD associated with a variance component is the standard deviation of the component divided by $\mu$. The variance components listed in the table are as follows: $\sigma_{C}$ is the standard deviation of $C_{i}$; $\sigma_{S}$ is the standard deviation of $S_{i j}$; and $\sigma_{E}$ is the analytical standard deviation.

Table B-1. Tank Concentration From Composite Samples. (8 pages)

\begin{tabular}{|c|c|c|c|c|c|c|c|c|}
\hline \multirow{2}{*}{ Analyte } & \multirow{2}{*}{$\begin{array}{l}\text { Analytical Method: } \\
\text { Sample Preparation }\end{array}$} & \multicolumn{2}{|c|}{ Mean Concentration } & \multicolumn{3}{|c|}{ ANOVA RSDs } & \multicolumn{2}{|c|}{ Obs. } \\
\hline & & $\overline{\dot{\mu}}$ & RSD $(\hat{\mu})$ & $\sigma_{c}$ & $\overline{\sigma_{c}}$ & $\overline{\sigma_{c}}$ & $<D L$ & $\#$ \\
\hline \multicolumn{9}{|c|}{ Anions } \\
\hline & & $(\mu g / g)$ & & & & & & \\
\hline Chloride & IC:W & $1.65 e+03$ & 7 & 10 & 0 & 3 & 0 & 8 \\
\hline Cyanide & IC:W & $3.49 e+00$ & 28 & 37 & 20 & 5 & 4 & 8 \\
\hline Fiuoride & IC:W & $5.83 e+03$ & 2 & 0 & 2 & 6 & 0 & 8 \\
\hline Nitrate & IC:W & $4.93 e+04$ & I & 0 & $\overline{0}$ & 3 & $\overline{0}$ & 8 \\
\hline Nitrite & IC:W & $8.81 \mathrm{e}+02$ & 13 & 18 & 6 & 6 & 0 & 8 \\
\hline Phosphate & IC:W & $1.21 \mathrm{e}+03$ & 15 & 21 & 0 & 3 & 0 & 8 \\
\hline Phosphate & ICP:F & $1.67 e+04$ & NA & NA & NA & NA & NA & NA \\
\hline SuIfate & IC:W & $3.480+02$ & 44 & 62 & NA & 1 & 4 & 8 \\
\hline
\end{tabular}


Table B-1. Tank Concentration From Composite Samples. (8 pages)

\begin{tabular}{|c|c|c|c|c|c|c|c|c|}
\hline \multirow{2}{*}{ Analyte } & \multirow{2}{*}{$\begin{array}{l}\text { Analytical Method: } \\
\text { Sample Preparation }\end{array}$} & \multicolumn{2}{|c|}{ Mean Concentration } & \multicolumn{3}{|c|}{ ANOVA RSDS } & \multicolumn{2}{|c|}{ Obs. } \\
\hline & & $\hat{\hat{\mu}}$ & RSD ( & $\sigma_{\mathrm{c}}$ & $\sigma_{c}$ & $\sigma_{\mathrm{c}}$ & $\angle \mathrm{DL}$ & $\#$ \\
\hline \multicolumn{9}{|c|}{ Cations } \\
\hline & & $(\mu g / g)$ & & & & & & \\
\hline Aluminum & ICP:A & $3.44 e+03$ & 74 & 102 & 30 & 27 & 0 & 13 \\
\hline Aluminum & ICP:F & $3.910+03$ & $7 !$ & 100 & 0 & 7 & 0 & 8 \\
\hline Aluminum & ICP:W & $5.83 e+01$ & 56 & 79 & 13 & 11 & 0 & 13 \\
\hline Ammonia & ISE:W & $1.04 e+01$ & $\overline{46}$ & $\overline{43}$ & $\overline{66}$ & 32 & 2 & 8 \\
\hline Antimony & $\mathrm{AA}(\mathrm{Sb}): \mathrm{A}$ & $3.88 e+01$ & NA & NA & NA & $\mathrm{NA}$ & 7 & 8 \\
\hline Antimony & ICP:A & $<3.71 e+01$ & NA & NA & NA & NA & 13 & 13 \\
\hline Antimony & ICP:F & $<2.40 e+02$ & NA & NA & NA & NA & 8 & 8 \\
\hline Antimony & ICP:W & $<8.04 \mathrm{e}+00$ & NA & NA & NA & NA & 13 & 13 \\
\hline Arsenic & AA (As):A & $4.50 e-01$ & 12 & 0 & $\overline{0}$ & 34 & 4 & 8 \\
\hline Arsenic & ICP:A & $5.95 e+01$ & NA & NA & NA & NA & 12 & 13 \\
\hline Arsenic & ICP:F & $4.11 e+02$ & 15 & 20 & 6 & 2 & 4 & 8 \\
\hline Arsenic & ICP:W & $<1.29 \mathrm{a}+01$ & NA & NA & NA & NA & 13 & 13 \\
\hline Barium & ICP:A & $8.64 e+01$ & 38 & 53 & 15 & 6 & 0 & 13 \\
\hline Barium & ICP:F & $1.67 e+02$ & 59 & 65 & 64 & 55 & 0 & 8 \\
\hline Barium & ICP:W & $<1.610+00$ & NA & NA & NA & NA & 13 & 13 \\
\hline Beryllium & ICP:A & $<3.71 c+00$ & NA & NA & NA & NA & 13 & 13 \\
\hline Beryllitum & ICP:F & $<2.40 \mathrm{e}+01$ & NA & NA & NA & NA & 8 & 8 \\
\hline Beryllium & ICP:W & $<8.04 c-01$ & NA & NA & NA & NA & 13 & 13 \\
\hline Bismuth & ICP:A & $1.01 \mathrm{e}+05$ & 13 & 17 & 0 & 15 & 0 & 13 \\
\hline Bismuth & ICP:F & $9.45 e+04$ & 3 & 0 & 6 & 4 & 0 & 8 \\
\hline Bismuth & ICP:W & 1.41e+01 & 22 & 30 & 0 & 20 & 6 & 13 \\
\hline Boron & ICP:A & $7.05 e+01$ & 35 & 49 & 5 & 18 & 0 & 13 \\
\hline Boron & ICP:F & $6.85 e+02$ & 87 & 102 & 58 & 112 & 3 & 8 \\
\hline Boron & ICP:W & $5.660+00$ & 41 & 0 & 78 & 39 & 7 & 13 \\
\hline Cadmium & ICP:A & $4.81 c+00$ & 6 & 0 & 5 & 18 & 8 & 13 \\
\hline Cadmium & ICP:F & $5.27 e+01$ & 58 & 61 & 78 & 23 & 4 & 8 \\
\hline Cadmium & ICP:W & $<8.04 \mathrm{e}-01$ & NA & NA & NA & NA & 13 & 13 \\
\hline Calcium & ICP:A & $1.22 \mathrm{e}+04$ & 58 & 80 & 27 & 15 & 0 & 13 \\
\hline Calcium & ICP:F & $1.04 e+04$ & 53 & 75 & 5 & 3 & 0 & 8 \\
\hline Calcium & ICP:W & $5.06 c+01$ & 66 & 52 & 109 & 15 & 0 & 13 \\
\hline Cerium & ICP:A & $6.96 \mathrm{e}+01$ & 11 & 12 & 0 & 27 & 9 & 13 \\
\hline Cerium & ICP:F & $<3.84 e+02$ & NA & NA & NA & NA & 8 & 8 \\
\hline Cerium & ICP:W & $<1.29 \mathrm{e}+0 \mathrm{I}$ & NA & NA & NA & $\mathrm{NA}$ & 13 & 13 \\
\hline Chromium & ICP:A & $3.34 \mathrm{e}+03$ & 4 & 5 & 0 & 9 & 0 & 13 \\
\hline Chromium & ICP:F & $3.38 \mathrm{e}+03$ & 8 & 11 & 4 & 5 & 0 & 8 \\
\hline Chromium & ICP:W & $8.59 e+02$ & 3 & 0 & 5 & 5 & 0 & 13 \\
\hline
\end{tabular}


Table B-1. Tank Concentration From Composite Samples. (8 pages)

\begin{tabular}{|c|c|c|c|c|c|c|c|c|}
\hline \multirow{2}{*}{ Analyte } & \multirow{2}{*}{$\begin{array}{l}\text { Analytical Method: } \\
\text { Sample Preparation }\end{array}$} & \multicolumn{2}{|c|}{ Mesin Concentration } & \multicolumn{3}{|c|}{ ANOVA RSDS } & \multicolumn{2}{|c|}{ Ohs. } \\
\hline & & $\overline{\boldsymbol{\mu}}$ & RSD $(\dot{\mu})$ & $\sigma_{c}$ & $\sigma_{\mathrm{c}}$ & $\sigma_{c}$ & $<D L$ & $\#$ \\
\hline Cobalt & ICP:A & $9.60 e+00$ & 4 & 0 & 0 & 16 & 8 & 13 \\
\hline Cobalt & ICP:F & $5.38 c+01$ & 8 & 0 & 5 & 20 & 5 & 8 \\
\hline Cobalt & ICP:W & $<1.61 \mathrm{e}+00$ & NA & NA & NA & NA & 13 & 13 \\
\hline Copper & ICP:A & $4.82 e+01$ & 67 & 94 & 0 & 31 & 0 & 13 \\
\hline Copper & ICP:F & $5.36 \mathrm{e}+01$ & 21 & 28 & 0 & 17 & 0 & 8 \\
\hline Copper & ICP:W & $<8.04 \mathrm{e}-01$ & NA & NA & NA & NA & 13 & 13 \\
\hline Dysprosium & ICP:A & $2.94 e+01$ & 31 & 38 & 0 & 56 & 9 & 13 \\
\hline Dysprositum & ICP:F & $<9.60 e+01$ & NA & NA & NA & NA & 8 & 8 \\
\hline Dysprosium & ICP:W & $<3.22 e+00$ & NA & NA & NA & NA & 13 & 13 \\
\hline Europium & ICP:A & $<7.43 e+00$ & NA & NA & NA & NA & 13 & 13 \\
\hline Europitm & ICP:F & $<4,80 e+01$ & NA & NA & NA & NA & 8 & 8 \\
\hline Europium & ICP:W & $<1.61 e+00$ & NA & NA & NA & NA & 13 & 13 \\
\hline Gadolinium & ICP:A & $1.69 e+02$ & 15 & 7 & 0 & 32 & 9 & 13 \\
\hline Gadolinium & ICP:F & $<9.60 e+02$ & NA & NA & $\mathrm{NA}$ & NA & 8 & 8 \\
\hline Gadolinium & ICP:W & $<3.22 \mathrm{e}+01$ & NA & NA & NA & NA & 13 & 13 \\
\hline Hexavalent Chromium & Calorimetric:W & $7.47 e+02$ & 6 & 7 & 5 & 7 & 0 & 8 \\
\hline Iron & ICP:A & $1.47 c+04$ & 32 & 44 & 5 & 7 & 0 & 13 \\
\hline Iron & ICP:F & $1.34 e+04$ & 20 & 28 & 6 & 0 & 0 & 8 \\
\hline Iron & ICP:W & $4.61 \mathrm{c}+00$ & 28 & 36 & 19 & 16 & 0 & 13 \\
\hline Lanthanum & ICP:A & $1.51 \mathrm{e}+04$ & 10 & 13 & 0 & 13 & 0 & 13 \\
\hline Lanthanum & ICP:F & $1.42 e+04$ & 4 & 0 & 7 & 4 & 0 & 8 \\
\hline Lanthanum & ICP:W & $1.99 e+01$ & 60 & 83 & 20 & 29 & 3 & 13 \\
\hline Lead & ICP:A & $1.36 \mathrm{e}+03$ & 9 & 12 & 2 & 12 & 0 & 13 \\
\hline Lead & ICP:F & $1.24 \mathrm{e}+03$ & 26 & 27 & 3 & 2 & 0 & 8 \\
\hline Lead & ICP:W & $<9.65 c+00$ & NA & NA & NA & NA & 13 & 13 \\
\hline Lithium & ICP:A & $<1.49 \mathrm{e}+01$ & NA & NA & NA & NA & 13 & 13 \\
\hline Lithium & ICP:F & $<9.60 e+01$ & NA & NA & NA & NA & 8 & 8 \\
\hline Lithium & ICP:W & $<3.22 \mathrm{e}+00$ & NA & $\mathrm{NA}$ & NA & NA & 13 & 13 \\
\hline Magnesium & ICP:A & $1.51 e+03$ & 58 & 80 & 24 & 13 & 0 & 13 \\
\hline Magnesium & ICP:F & $1.20 c+03$ & 48 & 64 & 33 & 2 & 0 & 8 \\
\hline Magnesium & ICP:W & $1.72 e+01$ & $\mathrm{NA}$ & NA & NA & NA & 11 & 13 \\
\hline Manganese & ICP:A & $1.92 e+04$ & 29 & 32 & 0 & 64 & 0 & 13 \\
\hline Manganese & ICP:F & $2.29 e+04$ & 6 & 8 & 6 & 4 & 0 & 8 \\
\hline Manganese & ICP:W & $3.15 e+\infty$ & 31 & 36 & 33 & 20 & 0 & 13 \\
\hline Mercury & CVAA:A & $5.99 c-01$ & 46 & 58 & 29 & 43 & 0 & 8 \\
\hline Motybdenum & ICP:A & $1.91 \mathrm{e}+01$ & 4 & 0 & 0 & 16 & 9 & 13 \\
\hline Molybdenum & ICP:F & $<9.60 e+01$ & NA & NA & NA & NA & 8 & 8 \\
\hline Molybdenum & ICP:W & $<3.22 e+00$ & NA & NA & NA & $\mathrm{NA}$ & 13 & 13 \\
\hline
\end{tabular}


Table B-1. Tank Concentration From Composite Samples. (8 pages)

\begin{tabular}{|c|c|c|c|c|c|c|c|c|}
\hline \multirow{2}{*}{ Analyte } & \multirow{2}{*}{$\begin{array}{l}\text { Analytical Method: } \\
\text { Sample Preparation }\end{array}$} & \multicolumn{2}{|c|}{ Mean Concentration } & \multicolumn{3}{|c|}{ ANOVA RSDs } & \multicolumn{2}{|c|}{ Obs. } \\
\hline & & $\overline{\dot{\mu}}$ & RSD (â) & $\sigma_{\mathrm{c}}$ & $\sigma_{\mathrm{c}}$ & $\sigma_{\mathbf{C}}$ & $<\mathrm{DL}$ & $\#$ \\
\hline Neodymium & ICP:A & $<2.23 e+01$ & NA & NA & NA & NA & 13 & 13 \\
\hline Neodymium & ICP:F & $<1.44 \mathrm{e}+02$ & NA & NA & NA & NA & 8 & $\overline{8}$ \\
\hline Neodymium & ICP:W & $<4.83 e+00$ & NA & NA & NA & NA & 13 & 13 \\
\hline Nickel & $\mathrm{ICP}: \mathrm{A}$ & $4.79 e+02$ & 3 & 0 & 0 & III & 0 & 13 \\
\hline Nickel & ICP:F & $4.290+02$ & 21 & NA & NA & 22 & 0 & 4 \\
\hline Nickel & ICP:W & $4.03 e+00$ & 24 & $\overline{33}$ & 0 & 22 & 9 & 13 \\
\hline Palladium & ICP:A & $<1.11 \mathrm{e}+02$ & $\mathrm{NA}$ & NA & NA & NA & 13 & 13 \\
\hline Palladium & ICP:F & $<7.20 \mathrm{e}+02$ & NA & NA & NA & $\overline{N A}$ & 8 & $\overline{8}$ \\
\hline Palladium & ICP:W & $<2,41 e+01$ & NA & NA & NA & NA & 13 & 13 \\
\hline Phosphorus & ICP:A & $5.79 \mathrm{e}+03$ & 20 & 28 & 10 & 6 & 0 & 13 \\
\hline Phosphorus & ICP:F & $5.45 e+03$ & 14 & 18 & 10 & 4 & 0 & 8 \\
\hline Phosphorus & ICP:W & $4.13 e+02$ & 17 & 24 & 4 & 7 & 0 & 13 \\
\hline Potassium & ICP:A & $5.81 e+03$ & 13 & 17 & 9 & 6 & 0 & 13 \\
\hline Potassium & ICP:F & $8.24+03$ & 7 & NA & NA & 14 & 0 & 4 \\
\hline Potassium & ICP:W & $4.65 e+03$ & 8 & 10 & 6 & 5 & 0 & 13 \\
\hline Rhodium & ICP:A & $<7.43 \mathrm{e}+0 \mathrm{I}$ & NA & NA & NA & NA & 13 & 13 \\
\hline Rhodium & ICP:F & $<4.80 e+02$ & NA & NA & NA & NA & 8 & 8 \\
\hline Rhodium & ICP:W & $<1.61 e+01$ & NA & NA & NA & NA & 13 & 13 \\
\hline Ruthenium & ICP:A & $<3.71 \mathrm{e}+01$ & NA & NA & NA & NA & 13 & 13 \\
\hline Ruthenium & ICP:F & $<2.40 \mathrm{e}+02$ & NA & NA & NA & NA & 8 & 8 \\
\hline Ruthenium & ICP:W & $<8.04 c+\infty 0$ & NA & $\overline{\text { NA }}$ & NA & NA & 13 & 13 \\
\hline Selenium & $\mathrm{AA}(\mathrm{Se}): \mathrm{A}$ & $<1.53 e+\infty$ & NA & NA & $\overline{N A}$ & NA & 8 & 8 \\
\hline Selenium & ICP:A & $6.68 e+01$ & 9 & 8 & 0 & 24 & 9 & 13 \\
\hline Selenium & ICP:F & $<3.60 \mathrm{e}+02$ & NA & NA & NA & NA & 8 & 8 \\
\hline Selenium & ICP:W & $<1.21 e+01$ & NA & NA & NA & NA & 13 & 13 \\
\hline Silicon & ICP:A & $2.42 e+03$ & 21 & 29 & 7 & 4 & 0 & 13 \\
\hline Silicon & ICP:F & $2.02 \mathrm{e}+04$ & 63 & 89 & 0 & 8 & 0 & 8 \\
\hline Silicon & ICP:W & $6.29 e+02$ & 29 & 38 & 20 & 12 & 0 & 13 \\
\hline Silver & ICP:A & $1.23 e+01$ & 20 & 27 & 9 & 14 & 5 & 13 \\
\hline Silver & ICP:F & $4.720+01$ & 7 & 6 & 7 & 13 & 6 & 8 \\
\hline Silver & ICP:W & $<1.61 \mathrm{e}+00$ & NA & NA & NA & NA & 13 & 13 \\
\hline Sodium & ICP:A & $3.79 e+04$ & 9 & 12 & 5 & 4 & 0 & 13 \\
\hline Sodium & ICP:F & $3.82 e+04$ & 2 & 0 & 0 & 6 & 0 & 8 \\
\hline Sodium & ICP:W & $3.09 \mathrm{e}+04$ & 3 & 2 & 4 & 4 & 0 & 13 \\
\hline Strontium & ICP:A & $9.23 e+02$ & 5 & 5 & 0 & 11 & 0 & 13 \\
\hline Strontium & ICP:F & $8.97 c+02$ & 6 & 7 & 6 & 3 & 0 & 8 \\
\hline Strontium & ICP:W & $9.38 \mathrm{e}-01$ & 9 & 0 & 17 & 3 & 1 & 13 \\
\hline Tellurium & ICP:A & $<7.43 e+01$ & NA & $\mathbf{N A}$ & NA & NA & 13 & 13 \\
\hline Tellutium & ICP:F & $<4.80 e+02$ & NA & NA & NA & NA & 8 & 8 \\
\hline Tellurium & ICP:W & $<1.61 \mathrm{e}+01$ & NA & NA & NA & NA & 13 & 13 \\
\hline
\end{tabular}


Table B-1. Tank Concentration From Composite Samples. (8 pages)

\begin{tabular}{|c|c|c|c|c|c|c|c|c|}
\hline \multirow{2}{*}{ Analyte } & \multirow{2}{*}{$\begin{array}{l}\text { Analytical Method: } \\
\text { Sample Preparation }\end{array}$} & \multicolumn{2}{|c|}{ Mean Concentration } & \multicolumn{3}{|c|}{ ANOVA RSDS } & \multicolumn{2}{|c|}{ Obs. } \\
\hline & & $\overline{\boldsymbol{\mu}}$ & $\operatorname{RSD}(\hat{\mu})$ & $\overline{\sigma_{\mathrm{c}}}$ & $\sigma_{c}$ & $\sigma_{\mathrm{c}}$ & $<\mathrm{DL}$ & $\#$ \\
\hline Thallium & ICP:A & $<3.710+02$ & NA & NA & NA & NA & 13 & 13 \\
\hline Thallium & ICP:F & $<2.40 e+03$ & NA & NA & NA & NA & 8 & 8 \\
\hline Thallium & ICP:W & $<8.04 e+01$ & NA & NA & NA & NA & 13 & 13 \\
\hline $\operatorname{Tin}$ & ICP:A & $5.960+02$ & NA & NA & NA & NA & 11 & 13 \\
\hline Tin & ICP:F & $<3.84 e+03$ & NA & NA & NA & NA & 8 & 8 \\
\hline Tin & ICP:W & $<1.29 e+02$ & NA & NA & NA & NA & 13 & 13 \\
\hline Titanium & ICP:A & $2.85 c+02$ & 75 & 104 & 29 & 27 & 0 & 13 \\
\hline Titanium & ICP:F & $4.25 \mathrm{e}+02$ & 68 & 96 & 0 & 3 & 0 & 8 \\
\hline Titanitum & ICP:W & $<8.04 c-01$ & NA & NA & NA & NA & 13 & 13 \\
\hline Tungsten & ICP:A & $5.96 \mathrm{e}+01$ & NA & NA & NA & NA & 12 & 13 \\
\hline Tungsten & ICP:F & $<3.84 c+02$ & NA & NA & NA & NA & 8 & 8 \\
\hline Tungsten & ICP:W & $<1.29 e+01$ & NA & NA & $\mathrm{NA}$ & NA & 13 & 13 \\
\hline Uranium & ICP:A & $8.78 e+02$ & 10 & 10 & 0 & 26 & 9 & 13 \\
\hline Uranium & ICP:F & $<4.80 e+03$ & NA & NA & NA. & NA & 8 & 8 \\
\hline Uranium & ICP:W & $<1.61 \mathrm{e}+02$ & NA & NA & NA & NA & 13 & 13 \\
\hline Uranium & Laser Fluorimetry:F & $1.56 e+02$ & 100 & 141 & 0 & 7 & 0 & 8 \\
\hline Vanadium & $\mathrm{ICP}: \mathrm{A}$ & $1.59 e+01$ & 38 & 52 & 21 & 15 & 5 & 13 \\
\hline Vanadium & ICP:F & $<4.80 \mathrm{e}+01$ & NA & NA & NA & NA & 8 & 8 \\
\hline Vanadium & ICP:W & $<1.61 \mathrm{e}+00$ & NA & NA & NA & NA & 13 & 13 \\
\hline Yttrium & ICP:A & $8.19 e+00$ & 18 & 21 & 0 & 37 & 9 & 13 \\
\hline Ynrium & ICP:F & $<4.80 \mathrm{e}+01$ & NA & NA & NA & NA & 8 & 8 \\
\hline Yttrium & ICP:W & $<1.610+00$ & NA & NA & NA & NA & 13 & 13 \\
\hline Zinc & ICP:A & $2.17 \mathrm{e}+02$ & 6 & 0 & 8 & 14 & 0 & 13 \\
\hline Zine & ICP:F & $2.32 e+02$ & 19 & 0 & 0 & 54 & 0 & 8 \\
\hline Zins & ICP:W & $<3.220+00$ & NA & NA & NA & NA & 13 & 13 \\
\hline Zirconium & ICP:A & $1.07 e+01$ & 10 & 0 & 18 & 15 & 7 & 13 \\
\hline Zirconium & ICP:F & $5.30 \mathrm{e}+01$ & 17 & 22 & 13 & 11 & 4 & 8 \\
\hline Zirconium & ICP:W & $<1.61 \mathrm{e}+00$ & NA & NA & NA & NA & 13 & 13 \\
\hline \multicolumn{9}{|c|}{ Organics } \\
\hline & & $(\mu g / g)$ & & & & & & \\
\hline 1,2,4-Trichlorobenzene & SVOA & $<4.80 \mathrm{e}+01$ & NA & NA & NA & NA & 8 & 8 \\
\hline 1,2-Dichtorobenzene & SVOA & $<4.800+01$ & NA & NA & NA & NA & 8 & 8 \\
\hline 1,3-Dichiorobenzene & SVOA & $<4.80 e+01$ & NA & NA & $\mathbf{N A}$ & NA & 8 & 8 \\
\hline 1,4-Dichlorobenzene & SVOA & $<4.80 \mathrm{e}+01$ & NA & NA & NA & NA & 8 & 8 \\
\hline 2,4,5-Trichlorophenol & SVOA & $<2.40 e+02$ & NA & NA & NA & NA & 8 & 8 \\
\hline 2,4,6-Trichlorophenol & SVOA & $<4.80 \mathrm{e}+0 \mathrm{t}$ & NA & NA & NA & NA & 8 & 8 \\
\hline 2,4-Dichlorophenol & SVOA & $<4.80 \mathrm{e}+0 \mathrm{t}$ & $\mathrm{NA}$ & NA & NA & NA & 8 & 8 \\
\hline 2,4-Dimethylphenol & SVOA & $<4.80 e+01$ & NA & NA & NA & NA & 8 & 8 \\
\hline 2,4-Dinitrophenol & SVOA & $<2.40 e+02$ & NA & NA & NA & NA & 8 & 8 \\
\hline
\end{tabular}


Table B-1. Tank Concentration From Composite Samples. (8 pages)

\begin{tabular}{|c|c|c|c|c|c|c|c|c|}
\hline \multirow{2}{*}{ Analyte } & \multirow{2}{*}{$\begin{array}{l}\text { Analytical Method: } \\
\text { Sample Preparation }\end{array}$} & \multicolumn{2}{|c|}{ Mean Concentration } & \multicolumn{3}{|c|}{ ANOVA RSDS } & \multicolumn{2}{|c|}{ Obs. } \\
\hline & & $\overline{\dot{\mu}}$ & $\operatorname{RSD}(\hat{\mu})$ & $\sigma_{\mathrm{c}}$ & $\sigma_{c}$ & $\sigma_{c}$ & $<\mathrm{DL}$ & $\#$ \\
\hline 2,4-Dinitrotoluene & SVOA & $<4.80 \mathrm{e}+01$ & NA & NA & NA & NA & 8 & 8 \\
\hline 2,6-Dinitrotoluene & SVOA & $<4.80 \mathrm{e}+0 \mathrm{I}$ & NA & NA & NA & $\mathrm{NA}$ & 8 & 8 \\
\hline 2-Chloronaphthalene & SVOA & $<4.80 e+01$ & NA & NA & NA & NA. & 8 & 8 \\
\hline 2-Chlorophenol & SVOA & $<4.80 e+01$ & NA & NA & NA & NA & 8 & 8 \\
\hline 2-Methylnaphthalene & SVOA & $<4.80 e+01$ & NA & NA & NA & $\mathrm{NA}$ & 8 & 8 \\
\hline 2-Methylphenol & SVOA & $<4.80 e+01$ & NA & NA & NA & $\mathrm{NA}$ & 8 & 8 \\
\hline 2-Nitroaniline & SVOA & $<2.40 c+02$ & NA & NA & NA & NA & 8 & 8 \\
\hline 2-Nitrophenol & SVOA & $<4.80 \mathrm{e}+01$ & NA & NA & $\mathrm{NA}$ & NA & 8 & 8 \\
\hline 3,3'-Dichlorobenzidine & SVOA & $<9.69 \mathrm{e}+01$ & NA & NA & NA & NA & 8 & 8 \\
\hline 3-Nitroaniline & SVOA & $<2.40 e+02$ & $\mathbf{N A}$ & NA & NA & NA & 8 & 8 \\
\hline 4,6-Dinjtro-0-cresol & SVOA & $<2.40 \mathrm{e}+02$ & NA & NA & NA & NA & 8 & 8 \\
\hline 4-Bromophenylphenyl ether & SVOA & $<4.80 \mathrm{e}+01$ & NA & NA & $\mathrm{NA}$ & NA & 8 & 8 \\
\hline 4-Chloro-3-methylphenol & SVOA & $<4.80 \mathrm{e}+01$ & NA & NA & NA & NA & 8 & 8 \\
\hline 4-Chioroaniline & SVOA & $<4.80 \mathrm{e}+01$ & NA & NA & NA & NA & 8 & 8 \\
\hline 4-Methylphenol & SVOA & $<4,80 \mathrm{e}+01$ & NA & NA & NA & NA & 8 & 8 \\
\hline 4-Nitroaniline & SVOA & $<2.40 e+02$ & NA & $\mathrm{NA}$ & NA & NA & 8 & 8 \\
\hline 4-Nitrophenol & SVOA & $<2.40 e+02$ & $\mathrm{NA}$ & NA & NA & NA & 8 & 8 \\
\hline Acenaphthene & SVOA & $<4.80 \mathrm{e}+01$ & NA & NA & NA & NA & 8 & 8 \\
\hline Acenaphthylene & SVOA & $<4.80 c+01$ & NA & $\mathbf{N A}$ & NA & NA & 8 & 8 \\
\hline Anthracene & SVOA & $<4.80 e+01$ & NA & NA & NA & NA & 8 & 8 \\
\hline Benzo(a)anthracene & SVOA & $<4.80 \mathrm{e}+01$ & NA & NA & NA & NA & 8 & 8 \\
\hline Benzo(a)pyrene & SVOA & $<4.80 c+01$ & NA & NA & NA & NA & 8 & 8 \\
\hline Benzo(b)fluoranthene & SVOA & $<4.80 \mathrm{e}+0 \mathrm{I}$ & NA & NA & NA & $\mathrm{NA}$ & 8 & 8 \\
\hline Benzo(ghi)perylene & SVOA & $<4.80 \mathrm{e}+01$ & NA & $\mathrm{NA}$ & NA & NA & 8 & 8 \\
\hline Benzo(k)fluoranthene & SVOA & $<4.80 e+01$ & NA & NA & NA & NA & 8 & 8 \\
\hline Benzoic acid & SVOA & $<2.40 e+02$ & NA & NA & NA & NA & 8 & 8 \\
\hline Benzyl alcohol & SVOA & $<4.80 c+01$ & NA & NA & NA & NA & 8 & 8 \\
\hline Bis(2-chloroethyl) ether & SVOA & $<4.80 e+01$ & NA & NA & NA & NA & 8 & 8 \\
\hline Bis(2-chloroisopropyl) & SVOA & $<4.80 e+01$ & NA & NA & NA & NA & 8 & 8 \\
\hline Butylbenzylphthalate & SVOA & $<4.80 \mathrm{e}+01$ & NA & NA & NA & NA & 8 & 8 \\
\hline Chrysene & SVOA & $<4.80 \mathrm{e}+0 \mathrm{t}$ & NA & NA & NA & NA & 8 & 8 \\
\hline Di-n-butylphthalate & SVOA & $<4.800+01$ & NA & NA & NA & NA & 8 & 8 \\
\hline Di-n-octylphthalate & SVOA & $<4.80 \mathrm{e}+01$ & NA & NA & NA & NA & 8 & 8 \\
\hline Dibenz [a,h]anthracene & SVOA & $<4.80 \mathrm{e}+01$ & NA & NA & NA & NA & 8 & 8 \\
\hline Dibenzofuran & SVOA & $<4.80 \mathrm{e}+01$ & NA & NA & NA & NA & 8 & 8 \\
\hline Diethylphuhalate & SVOA & $<4.80 \mathrm{e}+01$ & NA & NA & NA & NA & 8 & 8 \\
\hline Dimethyl phrhalate & SVOA & $<4.80 \mathrm{e}+01$ & NA & NA & NA & NA & 8 & 8 \\
\hline Dodecane & SVOA & $2.85 e+02$ & 8 & 0 & 11 & 17 & 0 & 8 \\
\hline Fluoranthene & SVOA & $<4.80 e+01$ & NA & NA & NA & NA & 8 & 8 \\
\hline Fluorene & SVOA & $<4.80 c+01$ & NA & NA & NA & NA & 8 & 8 \\
\hline
\end{tabular}

\section{B-8}


Table B-1. Tank Concentration From Composite Samples. (8 pages)

\begin{tabular}{|c|c|c|c|c|c|c|c|c|}
\hline \multirow{2}{*}{ Analyte } & \multirow{2}{*}{$\begin{array}{l}\text { Analytical Method: } \\
\text { Sample Preparation }\end{array}$} & \multicolumn{2}{|c|}{ Mean Concentration } & \multicolumn{3}{|c|}{ ANOVA RSDS } & \multicolumn{2}{|c|}{ Obs. } \\
\hline & & $\bar{\mu}$ & RSD $(\hat{\mu})$ & $\overline{\sigma_{c}}$ & $\sigma_{c}$ & $\sigma_{\mathrm{c}}$ & $<D L$ & $\#$ \\
\hline Hexachlorobenzene & SVOA & $<4.80 \mathrm{e}+01$ & NA & NA & NA & NA & 8 & 8 \\
\hline Hexachlorobutadiene & SVOA & $<4.80 e+01$ & NA & NA & NA & NA & 8 & $\overline{8}$ \\
\hline Hexnchloroethane & SVOA & $<4.80 \mathrm{e}+01$ & NA & NA & NA & NA & 8 & 8 \\
\hline Indeno(1,2,3-cd)pyrene & SVOA & $<4.80 c+01$ & NA & NA & NA & NA & 8 & 8 \\
\hline Isophorone & SVOA & $<4.80 \mathrm{e}+01$ & NA & $\overline{\text { NA }}$ & NA & NA & 8 & $\overline{8}$ \\
\hline N-Nitrosodiphenylamine & SVOA & $<4.80 \mathrm{e}+01$ & NA & NA & NA & NA & 8 & 8 \\
\hline Naphthalene & SVOA & $<4.800+01$ & NA & NA & NA & NA & 8 & 8 \\
\hline Nitrobenzene & SVOA & $<4.80 e+01$ & NA & NA & NA & NA & 8 & 8 \\
\hline Pentachlorophenol & SVOA & $<2.40 e+02$ & NA & NA & $\mathrm{NA}$ & NA & 8 & 8 \\
\hline Pentadecane & SVOA & $4.10 e+01$ & 37 & 50 & 19 & 11 & 0 & 8 \\
\hline Phenanthrene & SVOA & $<4.80 \mathrm{e}+01$ & NA & $\mathrm{NA}$ & NA & NA & 8 & 8 \\
\hline Phenol & SVOA & $<4.80 c+01$ & NA & NA & NA & NA & 8 & 8 \\
\hline Pyrene & SVOA & $<4.80 \mathrm{e}+01$ & NA & NA & NA & NA & 8 & 8 \\
\hline Tetradecane & SVOA & $1.03 e+03$ & 28 & 38 & 15 & 13 & 0 & 8 \\
\hline Total carbon & Persulfate Oxidation:D & $5.59 e+03$ & 50 & 71 & 0 & 8 & 0 & 8 \\
\hline Total carbon & Persulfate Oxidation:W & $2.55 e+03$ & 12 & 16 & 8 & 5 & 0 & 8 \\
\hline Total inorganic carbon & Persulfate Oxidation:D & $3.14 e+03$ & 32 & 45 & 7 & 3 & 0 & 8 \\
\hline Total inorganic carbon & Persulfate Oxidation:W & $2.09 e+03$ & 16 & 21 & 11 & 10 & 0 & 8 \\
\hline Total organic carbon & Persulfate Oxidation:D & $2.36 e+03$ & 73 & 103 & 8 & 21 & 0 & 8 \\
\hline Total organic carton & Total Organic Halides & $6.28 e+02$ & 9 & NA & NA & 18 & 0 & 4 \\
\hline Tridecane & SVOA & $9.290+02$ & 7 & 0 & 9 & 15 & 0 & 8 \\
\hline \multicolumn{9}{|c|}{ Physical Properties } \\
\hline & & (\%) & & & & & & \\
\hline Weight percent solids & Pereent Solids:D & $3.93 e+01$ & 3 & NA & NA & 8 & 0 & 8 \\
\hline \multicolumn{9}{|c|}{ Radionuclides } \\
\hline & & $(\mu \mathrm{Ci} / \mathrm{g})$ & & & & & & \\
\hline Americium-24I & Alpha Radchem:F & $2.87 \mathrm{e}-02$ & 4 & 0 & 0 & 11 & 0 & 8 \\
\hline Americium-241 & GEA:F & $3.10 \mathrm{e}-02$ & 4 & 0 & 3 & 11 & 0 & 8 \\
\hline Carbon-14 & Liq Scintillation:W & $3.16 e-04$ & NA & NA & NA & NA & 7 & 8 \\
\hline Cesium-134 & GEA:F & $2.38 \mathrm{e}-03$ & NA & NA & NA & NA & 0 & 3 \\
\hline Cesium-137 & GEA:F & $8.00 \mathrm{e}-01$ & 27 & 27 & 27 & 34 & 0 & 8 \\
\hline Cesium-137 & GEA:W & $4.69 \mathrm{e}-02$ & NA & NA & NA & NA & 0 & 4 \\
\hline Cobalt- 60 & GEA:F & $1.96 \mathrm{e}-03$ & 75 & 105 & 0 & 29 & 3 & 8 \\
\hline Curium-243/244 & Alpha Radchem:F & $1.64 \mathrm{e}-03$ & 21 & $\mathrm{NA}$ & NA & 41 & 0 & 4 \\
\hline Europium-154 & GEA:F & $4.38 c-03$ & 51 & 67 & 37 & 13 & 2 & 8 \\
\hline Europium-155 & GEA:F & $3.28 c-03$ & NA & NA. & NA & NA & 4 & 5 \\
\hline Gross alpha & Alpha Radchem:F & $1.31 e+00$ & 18 & 26 & 0 & 5 & 0 & 8 \\
\hline Gross alpha & Alpha Radichem:W & $4.71 \mathrm{e}-04$ & 21 & NA & NA & 30 & 0 & 4 \\
\hline Gross beta & Beta Radehem:F & $4.41 e+00$ & 28 & 25 & 0 & 62 & 0 & 8 \\
\hline Gross beta & Beta Radchern:W & $5.34 \mathrm{e}-02$ & 16 & NA & NA & 4 & 0 & 4 \\
\hline
\end{tabular}


Table B-1. Tank Concentration From Composite Samples. (8 pages)

\begin{tabular}{|c|c|c|c|c|c|c|c|c|}
\hline \multirow{2}{*}{ Aralyte } & \multirow{2}{*}{$\begin{array}{l}\text { Analytical Method: } \\
\text { Sample Preparation }\end{array}$} & \multicolumn{2}{|c|}{ Mean Concentration } & \multicolumn{3}{|c|}{ ANOVA RSDS } & \multicolumn{2}{|c|}{ Ohs. } \\
\hline & & $\hat{\hat{A}}$ & RSD $(\hat{\mu})$ & $\sigma_{c}$ & $\sigma_{c}$ & $\sigma_{c}$ & $<D L$ & $\#$ \\
\hline Neptunium-237 & Alpha Radchem:F & $<1.24 \mathrm{e}-04$ & NA & NA & NA & NA & 8 & 8 \\
\hline Nickel-59 & Beta Radchem:A & $6.86 \mathrm{e}-06$ & 22 & 29 & 0 & 25 & 6 & 8 \\
\hline Nickel-63 & Liq Scintillation:A & $1.88 \mathrm{e}-04$ & 25 & 0 & 0 & 71 & 0 & 8 \\
\hline Plutonium-238 & Alpha Radchem:F & $6.90 e-03$ & 98 & 139 & 0 & 16 & 0 & 4 \\
\hline Plutonium-239/240 & Alpha Radchem:F & $1.13 e+00$ & 30 & 42 & 0 & 8 & 0 & 4 \\
\hline Potassium-40 & GEA:W & $1.24 \mathrm{e}-03$ & NA & NA & NA & NA & 0 & 2 \\
\hline Strontium-90 & Beta Radchem:F & $2.09 \mathrm{e}+00$ & 51 & 68 & 32 & 12 & 0 & 8 \\
\hline Technetium-99 & Beta Radchem:F & $1.94 \mathrm{e}-03$ & NA & NA & NA & $\mathrm{NA}$ & 8 & 8 \\
\hline Total alpha* & Alpha Radehem:F & $1.140+00$ & $\overline{\mathbf{N A}}$ & NA & $\overline{\text { NA }}$ & NA & 0 & 4 \\
\hline Tritium & Liq Scintillation:W & $2.05 e-02$ & 78 & 108 & 33 & 7 & 0 & 8 \\
\hline & & (\%) & & & & & & \\
\hline Uranium-234 & Mass Spectrometry:F & $5.40 \mathrm{e}-03$ & 6 & 0 & 0 & 17 & 0 & 8 \\
\hline Uranium-235 & Mass Spectrometry:F & $6.91 \mathrm{e}-01$ & 0 & 1 & 0 & $\overline{0}$ & 0 & 8 \\
\hline Uranium-236 & Mass Spectrometry:F & $5.20 \mathrm{e}-03$ & 6 & 2 & 0 & 16 & 0 & 8 \\
\hline Uranium-238 & Mass Spectrometry:F & $9.93 e+01$ & 0 & 0 & 0 & 0 & 0 & $\overline{8}$ \\
\hline Plutonium-238 & Mass Spectrometry:F & $4.87 e-03$ & 15 & 0 & 0 & 43 & 0 & 8 \\
\hline Plutonium-239 & Mass Spectrometry:F & $9.84 e+01$ & 0 & 0 & 0 & $\overline{0}$ & 0 & 8 \\
\hline Plutonium-240 & Mass Spectrometry:F & $1.55 \mathrm{e}+00$ & 1 & 1 & 0 & 2 & 0 & 8 \\
\hline Plutonium-241 & Mass Spectrometry:F & $1.30 \mathrm{e}-02$ & 16 & 0 & 0 & 46 & 0 & 8 \\
\hline Plutonium-242 & Mass Spectrometry:F & $3.84 \mathrm{e}-03$ & 43 & 42 & 50 & 4 & 0 & 8 \\
\hline
\end{tabular}

*Total alpha emitted from ${ }^{248} \mathrm{Pu},{ }^{205} \mathrm{Pu},{ }^{2 / 0} \mathrm{Pu},{ }^{241} \mathrm{Pu}$. 
WHC-SD-WM-ER-550 Rev 0

APPENDIX C

SEGMENT-LEVEL ESTIMATES AND VARIABILITY SUMMARY

C-1 
This page intentionally left blank. 


\section{APPENDIX C}

\section{SEGMENT-LEVEL ESTIMATES AND VARUABILITY SUMMARY}

This appendix contains estimates of concentration computed from segment-level data. One can also compute vertical profiles of the waste from the segment-level data. The basic results of the ANOVA fits are presented in the table, while the vertical profiles are presented graphically.

The model used to produce these results is:

$$
Y_{i j k}=\mu+C_{i}+S_{j}+C S_{i j}+E_{i j k}
$$

where $i$ is the core, $j$ is the segment, and $k$ is the sample replicate of the measurement. The term $\mu$ represents the tank average and the other terms represent deviations from the tank average. Each deviation in the above model has an associated variability that is measured by the ANOVA procedure. These sources of variability are denoted by $\sigma_{C}, \sigma_{S}$, etc., and are expressed as a percent RSD (the sigma divided by $\mu$ ).

Table C-1. Tank Concentrations from Segment Samples. ( 3 pages)

\begin{tabular}{|c|c|c|c|c|c|c|c|c|}
\hline \multirow[t]{2}{*}{ Constituent } & \multicolumn{2}{|c|}{ Mean Concentration } & \multicolumn{4}{|c|}{ ANOVA RSD's } & \multicolumn{2}{|c|}{ Obs. } \\
\hline & $\hat{\mu}$ & RSD & $\sigma_{c}$ & $\sigma_{s}$ & $\sigma_{c s}$ & $\sigma_{E}$ & $<D L$ & $\#$ \\
\hline \multicolumn{9}{|c|}{ Anions } \\
\hline Phosphorus & $4.71 \mathrm{e}+03 \quad \mu \mathrm{g} / \mathrm{g}$ & 12 & 0 & 13 & 13 & 4 & 0 & 24 \\
\hline \multicolumn{9}{|c|}{ Metals } \\
\hline Aluminum & $1.27 \mathrm{e}+03 \quad \mu \mathrm{g} / \mathrm{g}$ & 87 & 5 & 106 & 89 & 3 & 0 & 24 \\
\hline Barium & $6.13 \mathrm{e}+01 \quad \mu \mathrm{g} / \mathrm{g}$ & 9 & 6 & 0 & 15 & 6 & $\overline{0}$ & 24 \\
\hline Bismuth & $9.15 \mathrm{e}+04 \quad \mu \mathrm{g} / \mathrm{g}$ & 29 & 3 & 40 & 8 & 7 & 0 & 24 \\
\hline Boron & $4.01 \mathrm{e}+03 \quad \mu \mathrm{g} / \mathrm{g}$ & 91 & 0 & 32 & 177 & 6 & 0 & 24 \\
\hline Calcium & $6.38 \mathrm{e}+03 \quad \mu \mathrm{g} / \mathrm{g}$ & 75 & 0 & 103 & 35 & 4 & 0 & 24 \\
\hline Chromium & $3.34 \mathrm{e}+03 \quad \mu \mathrm{g} / \mathrm{g}$ & 30 & 15 & 35 & 23 & 4 & 0 & 24 \\
\hline Copper & $2.49 \mathrm{e}+01 \quad \mu \mathrm{g} / \mathrm{g}$ & 71 & 30 & 89 & 48 & 12 & 7 & 24 \\
\hline Iron & $1.13 \mathrm{e}+04 \mu \mathrm{g} / \mathrm{g}$ & 32 & 6 & 45 & 0 & 5 & 0 & 24 \\
\hline Lanthanum & $1.35 e+04 \quad \mu \mathrm{g} / \mathrm{g}$ & 25 & 0 & 34 & 12 & 4 & 0 & 24 \\
\hline Lead & $2.69 \mathrm{e}+02 \quad \mu \mathrm{g} / \mathrm{g}$ & 57 & 5 & 80 & 8 & 8 & 4 & 24 \\
\hline Magnesium & $7.84 \mathrm{e}+02 \mu \mathrm{g} / \mathrm{g}$ & 65 & 0 & 85 & 46 & 3 & 0 & 24 \\
\hline Manganese & $1.66 \mathrm{e}+04 \mu \mathrm{g} / \mathrm{g}$ & 33 & 33 & 26 & 0 & 68 & 0 & 24 \\
\hline Molybdenum & $3.09 \mathrm{e}+01 \quad \mu \mathrm{g} / \mathrm{g}$ & 26 & 34 & 8 & 0 & 36 & 20 & 24 \\
\hline Nickel & $4.31 \mathrm{e}+02 \quad \mu \mathrm{g} / \mathrm{g}$ & 27 & 19 & 33 & 0 & 5 & 0 & 24 \\
\hline
\end{tabular}


Table C-1. Tank Concentrations from Segment Samples. (3 pages)

\begin{tabular}{|c|c|c|c|c|c|c|c|c|c|}
\hline \multirow[t]{2}{*}{ Constituent } & \multicolumn{3}{|c|}{ Mean Concentration } & \multicolumn{4}{|c|}{ ANOVA RSD's } & \multicolumn{2}{|c|}{ Obs. } \\
\hline & \multicolumn{2}{|c|}{$\hat{\mu}$} & \multirow{2}{*}{$\begin{array}{r}\text { RSD } \\
15\end{array}$} & \multirow{2}{*}{$\sigma_{c}$} & \multirow{2}{*}{$\frac{\sigma_{s}}{20}$} & \multirow{2}{*}{$\begin{array}{r}\sigma_{c s} \\
0\end{array}$} & \multirow{2}{*}{$\frac{\sigma_{E}}{16}$} & \multirow{2}{*}{$\begin{array}{r}<\text { DL } \\
3\end{array}$} & \multirow{2}{*}{$\frac{\#}{24}$} \\
\hline Potassium & $2.90 e+03$ & $\mu \mathrm{g} / \mathrm{g}$ & & & & & & & \\
\hline Silicon & $5.07 e+03$ & $\mu g / g$ & 69 & 20 & 46 & 118 & 7 & 0 & 24 \\
\hline Silver & $1.52 \mathrm{e}+01$ & $\mu g / g$ & 28 & 36 & 12 & 0 & 37 & 20 & 24 \\
\hline Sodium & $3.40 e+04$ & $\mu g / g$ & 10 & 3 & 13 & 2 & 5 & 0 & 24 \\
\hline Strontium & $9.02 e+02$ & $\mu \mathrm{g} / \mathrm{g}$ & 31 & 0 & 44 & 7 & 4 & 0 & 24 \\
\hline Titanium & $1.14 e+02$ & $\mu g / g$ & 90 & 30 & 95 & 110 & 1 & 1 & 24 \\
\hline Uranium & $1.47 e+03$ & $\mu g / g$ & 33 & 40 & 14 & 17 & 41 & 21 & 24 \\
\hline Zine & $4.66 e+01$ & $\mu g / g$ & 31 & 17 & 40 & 0 & 10 & 7 & 24 \\
\hline Zirconium & $1.60 e+01$ & $\mu g / g$ & 23 & 13 & 16 & 35 & 31 & 20 & 24 \\
\hline \multicolumn{10}{|c|}{ Organies } \\
\hline Hexamethyl-disiloxane & $2.14 \mathrm{e}+04$ & $\mu \mathrm{g} / \mathrm{kg}$ & 26 & $\mathrm{NA}$ & 35 & NA & 35 & 0 & 5 \\
\hline Methoxytri-methylsilane & $1.43 e+05$ & $\mu \mathrm{g} / \mathrm{kg}$ & 22 & NA & 35 & $\mathrm{NA}$ & 24 & 0 & 6 \\
\hline Toluene & $5.08 \mathrm{e}+03$ & $\mu \mathrm{g} / \mathrm{kg}$ & 23 & NA & 38 & NA & 13 & 0 & 6 \\
\hline Trimethyl-silanol & $1.32 c+05$ & $\mu \mathrm{g} / \mathrm{kg}$ & 28 & NA & 43 & NA & 35 & 0 & 6 \\
\hline \multicolumn{10}{|c|}{ Physical Properties } \\
\hline Centrifuged solids density & $1.47 e+00$ & $\mathrm{~g} / \mathrm{ml}$ & 7 & NA & 9 & NA & 6 & 0 & 9 \\
\hline Centrifuged supernate density & $1.05 e+00$ & $\mathrm{~g} / \mathrm{ml}$ & 3 & NA & 0 & NA & 8 & 0 & 9 \\
\hline Critical flow rate-2in & $3.80 \mathrm{e}+01$ & $\mathrm{gal} / \mathrm{min}$ & 39 & NA & 54 & NA & 20 & 0 & 6 \\
\hline Critical flow rate-3in & $7.55 \mathrm{e}+01$ & $\mathrm{gal} / \mathrm{min}$ & 38 & NA & 53 & NA & 21 & 0 & 6 \\
\hline Critical velocity-2in & $3.66 \mathrm{e}+00$ & $\mathrm{ft} / \mathrm{sec}$ & 38 & NA & 52 & $\mathrm{NA}$ & 20 & 0 & 6 \\
\hline Critical velocity-3in & $3.27 e+00$ & $\mathrm{ft} / \mathrm{sec}$ & 38 & NA & 52 & NA & 21 & 0 & 6 \\
\hline Density & $1.25 \mathrm{e}+00$ & $\mathrm{~g} / \mathrm{ml}$ & 2 & 0 & 0 & 0 & 11 & 0 & 26 \\
\hline Flow behavior index & $8.62 e-01$ & UNITLESS & 4 & NA & 0 & NA & 11 & 0 & 6 \\
\hline Particle size number density mean & $1.26 \mathrm{e}+00$ & microns & 5 & NA & 0 & NA & 14 & 0 & 8 \\
\hline Particle size volume density mean & $2.92 e+01$ & microns & 21 & NA & 0 & NA & 60 & 0 & 8 \\
\hline Reynolds number-2in & $8.26 \mathrm{e}+03$ & UNITLESS & 1 & NA & 0 & NA & 3 & 0 & 6 \\
\hline Reynolds number-3in & $1.04 e+04$ & UNITLESS & 3 & NA & 0 & $\mathrm{NA}$ & 6 & 0 & 6 \\
\hline Sertled solids & $7.69 e+01$ & $\%$ & 11 & NA & 0 & NA & 33 & 0 & 9 \\
\hline Shear strength & $1.33 e+04$ & dynes $/ \mathrm{cm}^{2}$ & 5 & NA & 6 & NA & 5 & 0 & 3 \\
\hline Volume \% centrifuged solids & $5.42 e+01$ & $\%$ & 20 & NA & 0 & NA & 61 & 0 & 9 \\
\hline Weight loss \% & $4.33 e+01$ & $\%$ & 17 & NA & 17 & NA & 53 & 0 & 16 \\
\hline Weight $\%$ centrifuged solids & $5.93 e+01$ & $\%$ & 17 & NA & 0 & NA & 52 & 0 & 9 \\
\hline Weight \% solids & $4.64 e+01$ & $\%$ & 18 & 20 & 0 & 22 & 36 & 0 & 32 \\
\hline Yield point & $3.84 \mathrm{e}+00$ & $\mathbf{P a}$ & 55 & NA & 76 & NA & 24 & 0 & 6 \\
\hline
\end{tabular}


Table C-1. Tank Concentrations from Segment Samples. (3 pages)

\begin{tabular}{|c|c|c|c|c|c|c|c|c|}
\hline \multirow[t]{2}{*}{ Constituent } & \multicolumn{2}{|c|}{ Mean Concentration } & \multicolumn{4}{|c|}{ ANOVA RSD's } & \multicolumn{2}{|c|}{ Obs. } \\
\hline & $\hat{\mu}$ & RSD & $\sigma_{c}$ & $\sigma_{s}$ & $\sigma_{c s}$ & $\sigma_{E}$ & $<\mathrm{DL}$ & $\#$ \\
\hline \multicolumn{9}{|c|}{ Radionuclides } \\
\hline Americium-241 & $2.83 \mathrm{e}-02 \quad \mu \mathrm{Ci} / \mathrm{g}$ & 41 & 3 & 48 & 46 & 9 & 0 & 24 \\
\hline Cesium-134 & $1.62 e-03 \quad \mu \mathrm{Ci} / \mathrm{g}$ & 13 & 0 & 7 & 0 & 44 & 7 & 15 \\
\hline Cesium-137 & $1.45 \mathrm{e}-01 \quad \mu \mathrm{Ci} / \mathrm{g}$ & 57 & 17 & 78 & 0 & 42 & 0 & 24 \\
\hline Cobalt- 60 & $1.90 \mathrm{e}-03 \quad \mu \mathrm{Ci} / \mathrm{g}$ & 29 & 26 & 23 & 22 & 34 & $\overline{6}$ & 18 \\
\hline Europium-154 & $3.32 \mathrm{e}-03 \quad \mu \mathrm{Ci} / \mathrm{g}$ & 31 & 34 & 16 & 25 & 34 & 7 & 17 \\
\hline Europium- 155 & $4.15 \mathrm{e}-03 \quad \mu \mathrm{Ci} / \mathrm{g}$ & 23 & 26 & 15 & 0 & 25 & 8 & 12 \\
\hline
\end{tabular}

The pages that follow contain plots only for the best method of analysis. In other words, only our best estimate for each available constituent is presented. Each plot describes an individual analyte, and shows its vertical profile $\mu+S_{j}$ and confidence bounds, as well as the core profiles $\mu+C_{i}+S_{j}+C S_{i j}$ and their confidence bounds. 
Figure C-1. Plots for Best Method of Analysis. (page 1 of 14)

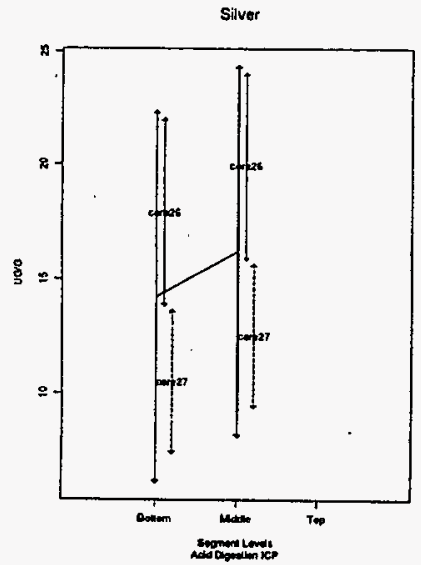

(1)

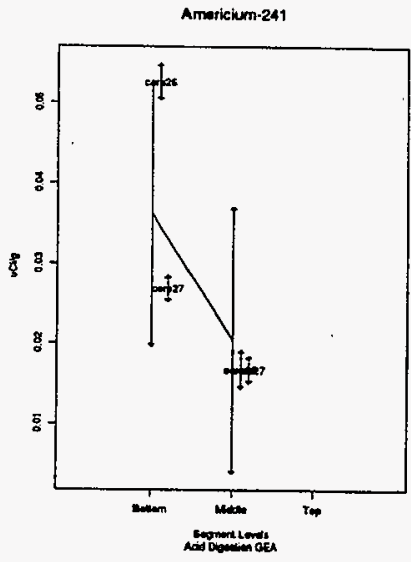

(3)

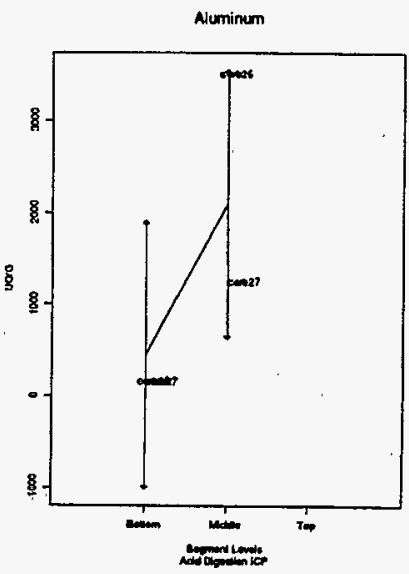

(2)

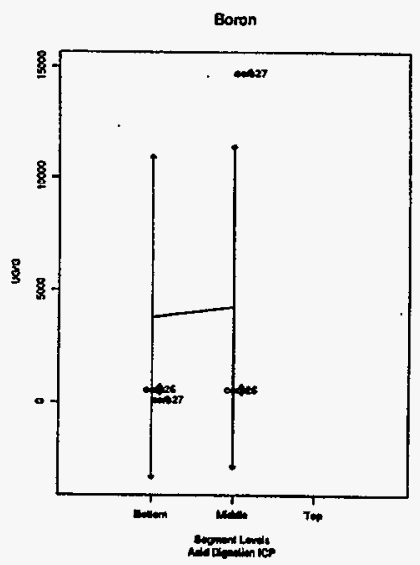

(4) 
Figure C-1. Plots for Best Method of Analysis. (page 2 of 14)

Bayium

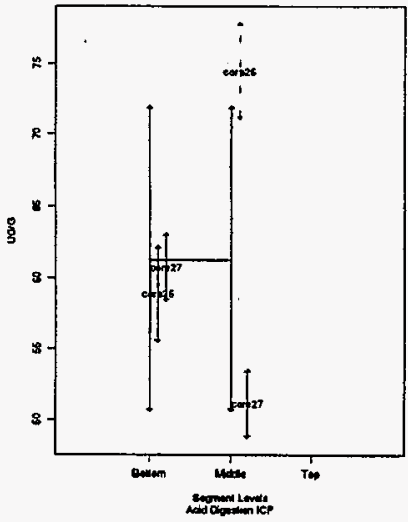

(5)

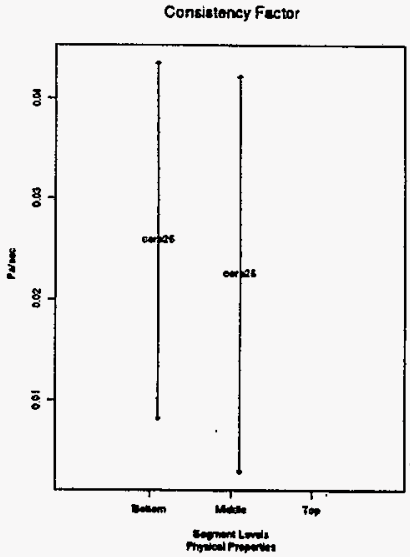

(7)

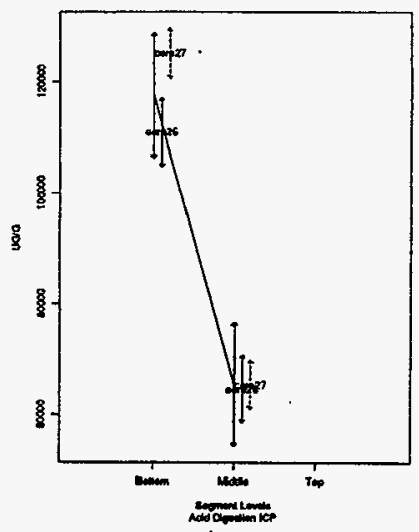

(6)

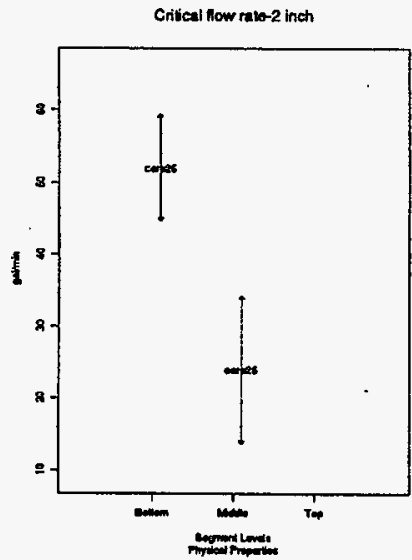

(8) 
Figure C-1. Plots for Best Method of Analysis. (page 3 of 14)

Critical flow rate- 3 inch

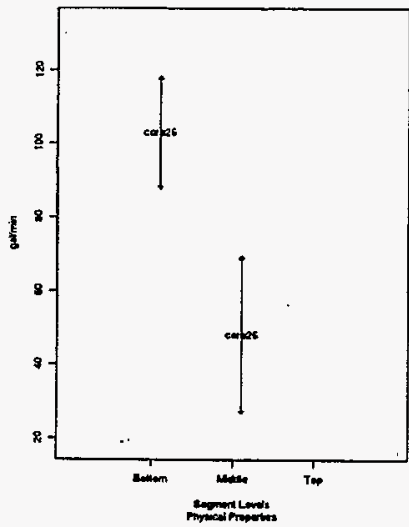

(9)

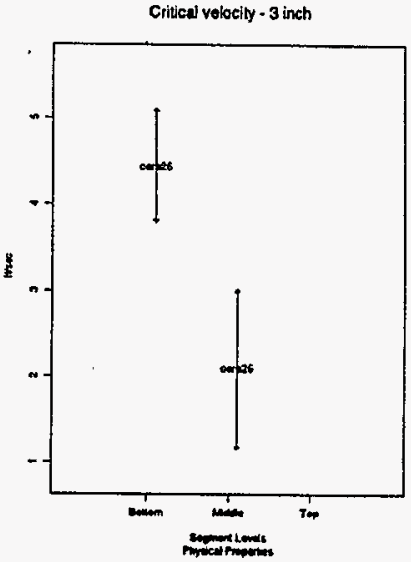

(11)
Critical velocity -2 inch

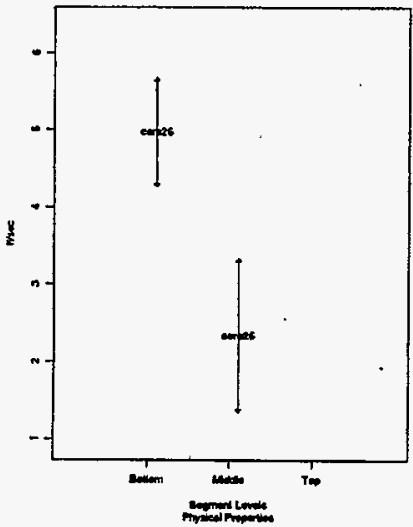

(10)

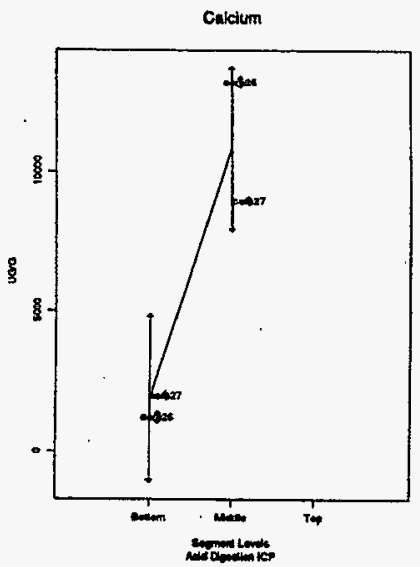

(12) 
Figure C-1. Plots for Best Method of Analysis. (page 4 of 14)

Cosium-134

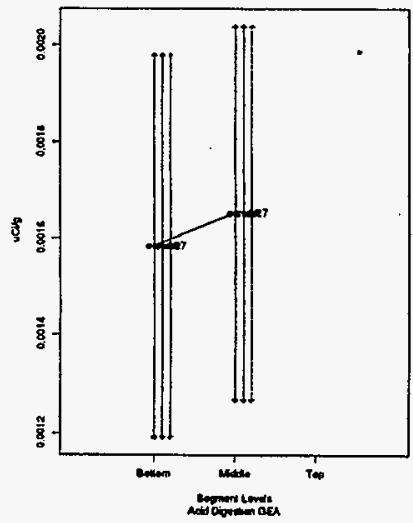

(13)

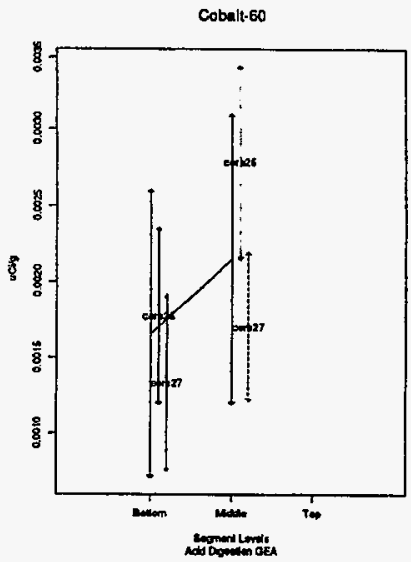

(15)
Corium-137

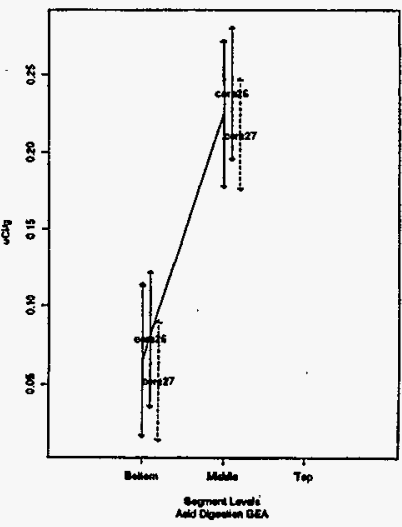

(14)

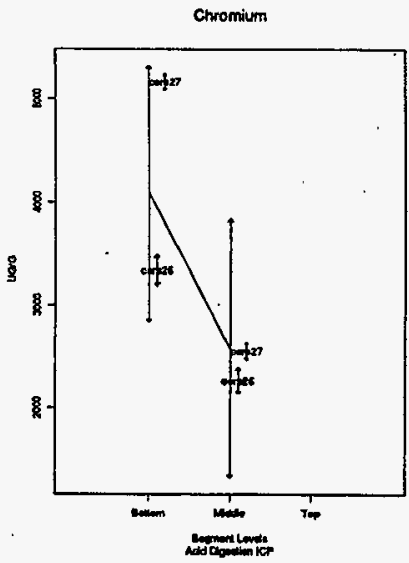

(16) 
Figure C-1. Plots for Best Method of Analysis. (page 5 of 14)

Copper

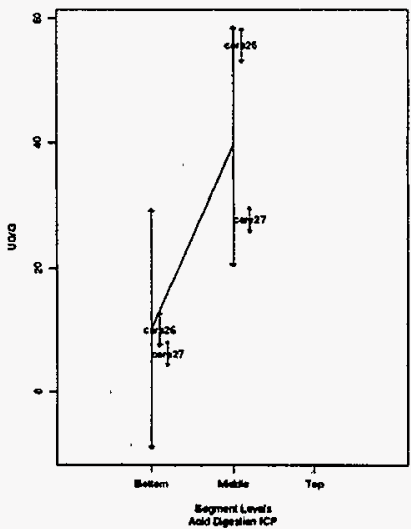

(17)

Europium*155

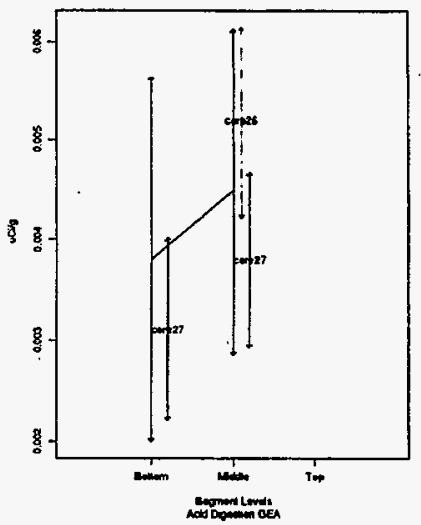

(19)
Europiun-154

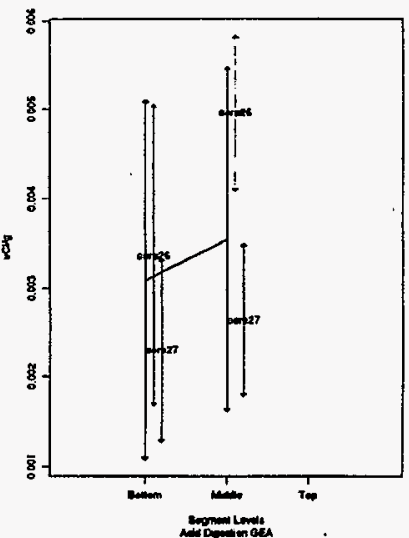

(18)

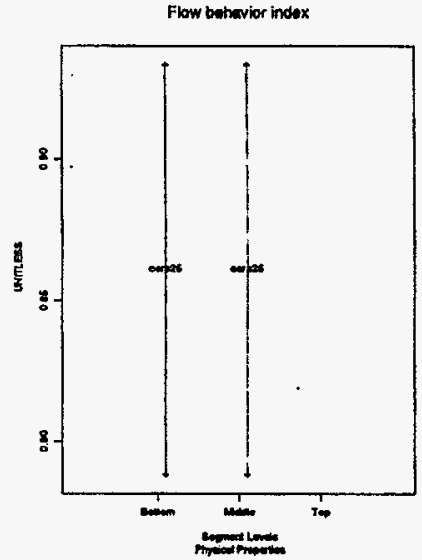

(20) 
Figure C-1. Plots for Best Method of Analysis. (page 6 of 14)

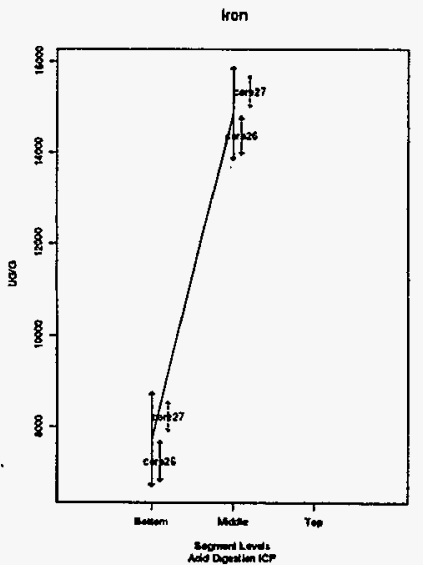

(21)

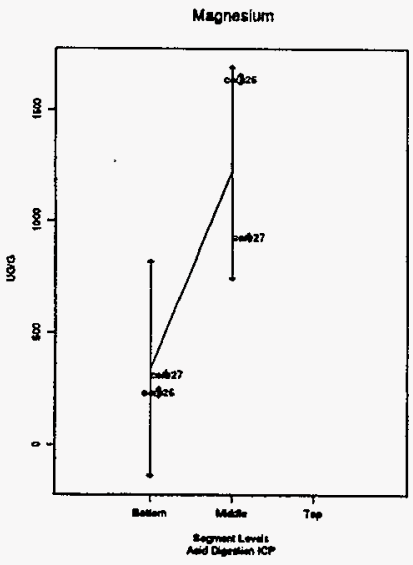

(23)

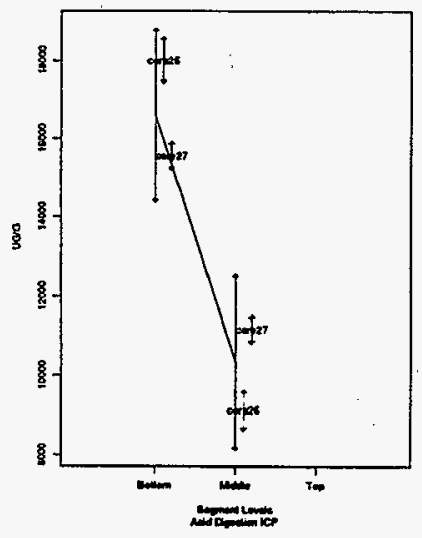

(22)

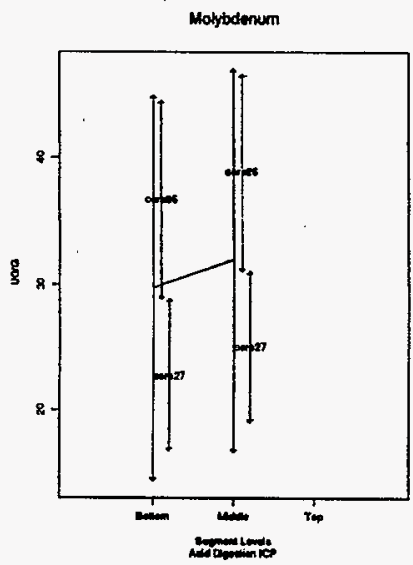

(24) 
Figure C-1. Plots for Best Method of Analysis. (page 7 of 14)

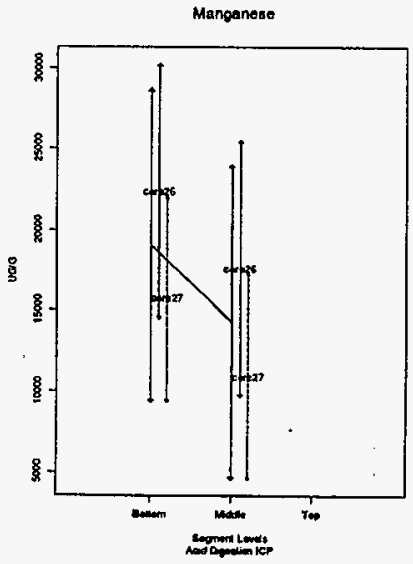

(25)

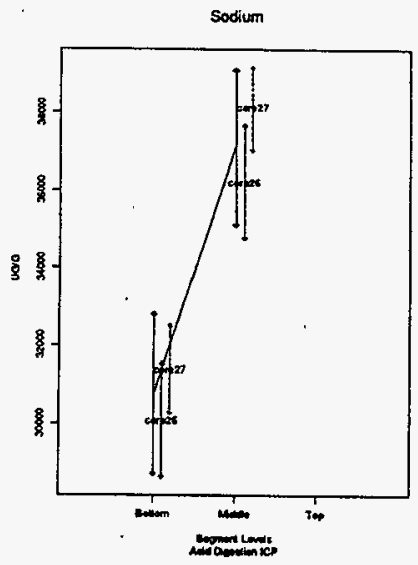

(27)
Methoxytrimethyisilane

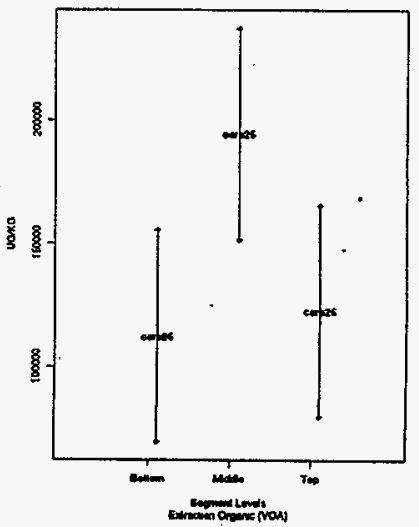

(26)

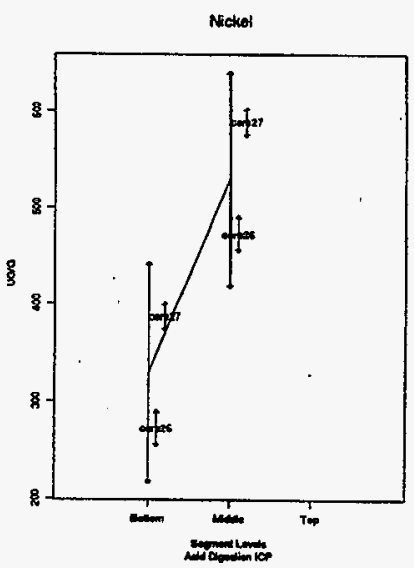

(28) 
Figure C-1. Plots for Best Method of Analysis. (page 8 of 14)

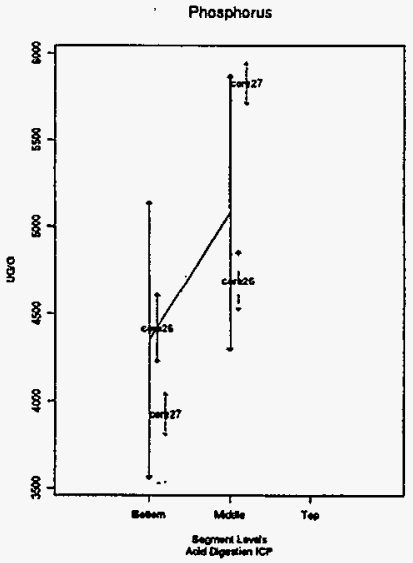

(29)

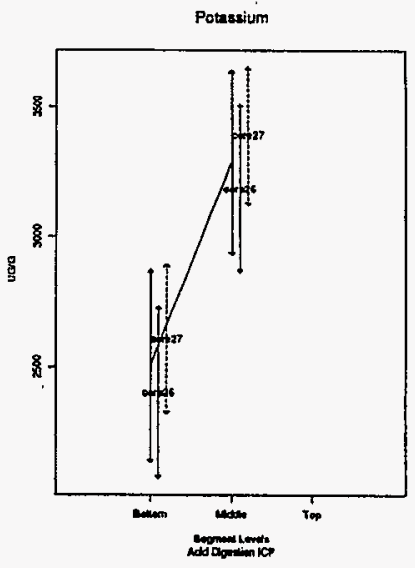

(31)

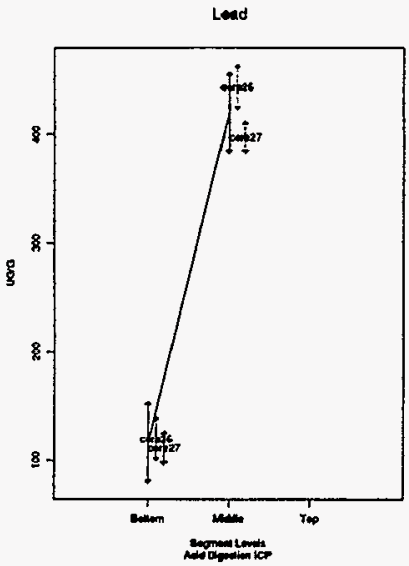

(30)

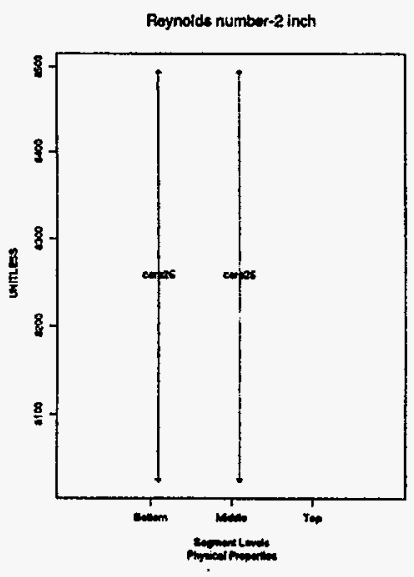

(32) 
Figure C-1. Plots for Best Method of Analysis. (page 9 of 14)

Reynolds number-3 inch

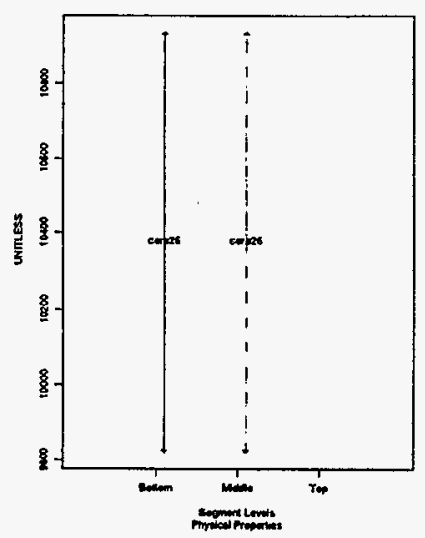

(33)

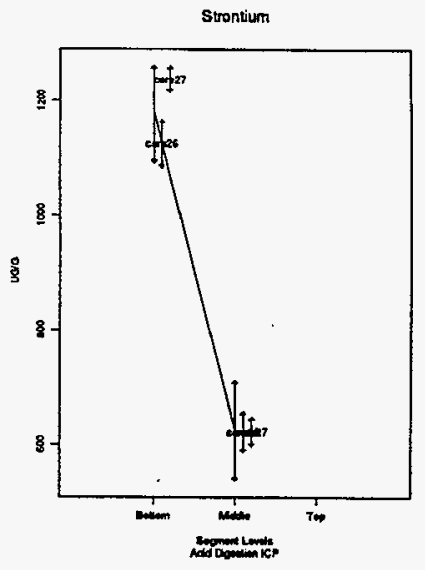

(35)

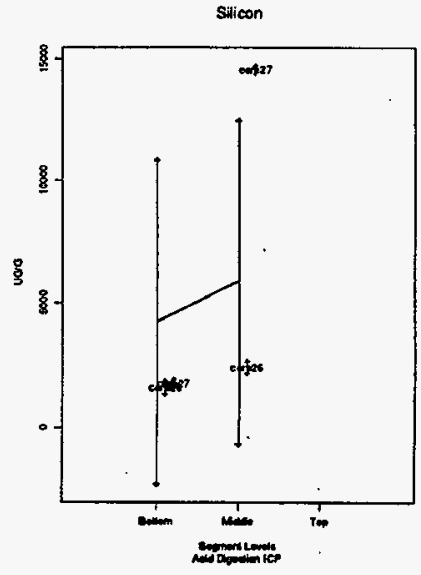

(34)

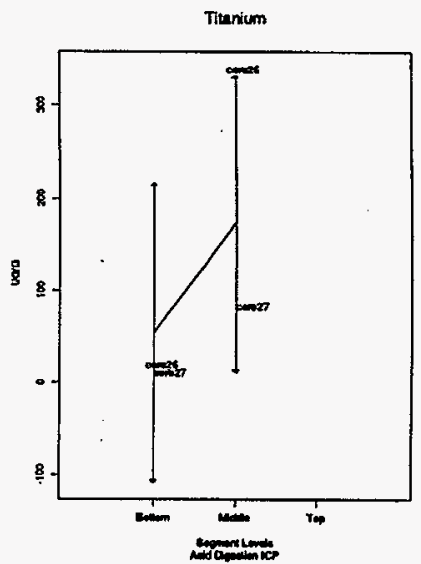

(36) 
Figure C-1. Plots for Best Method of Analysis. (page 10 of 14)

Trimethylsilanol Uranium

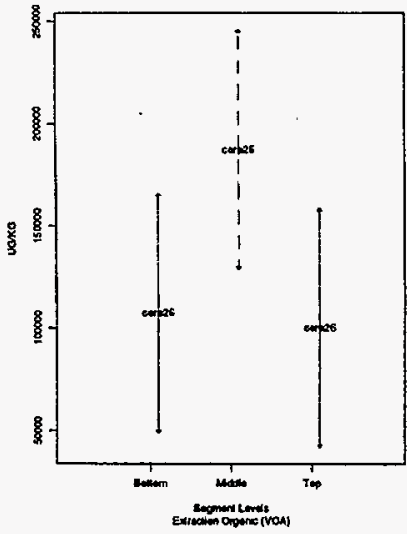

(37)

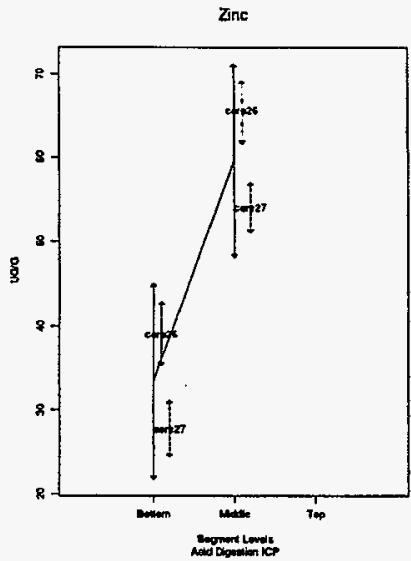

(39)

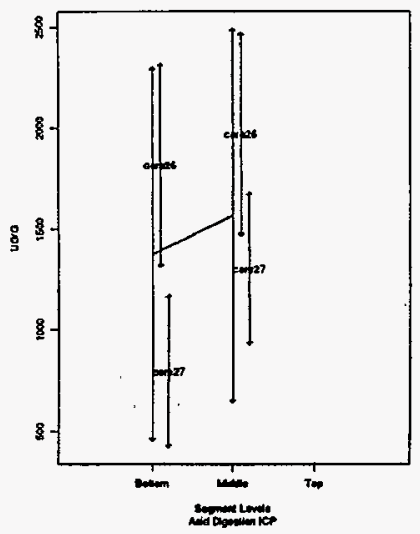

(38)

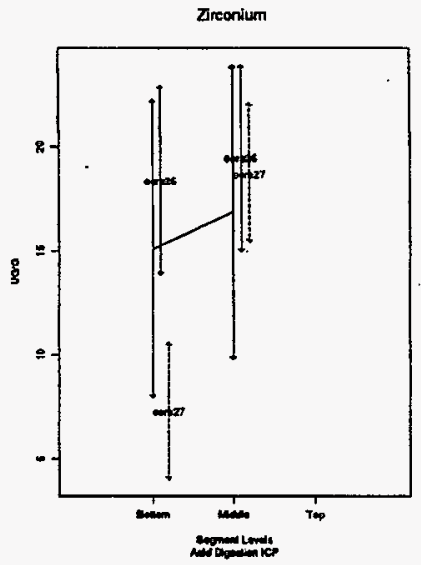

(40) 
Figure C-1. Plots for Best Method of Analysis. (page 11 of 14)

Centrifuged soljds density

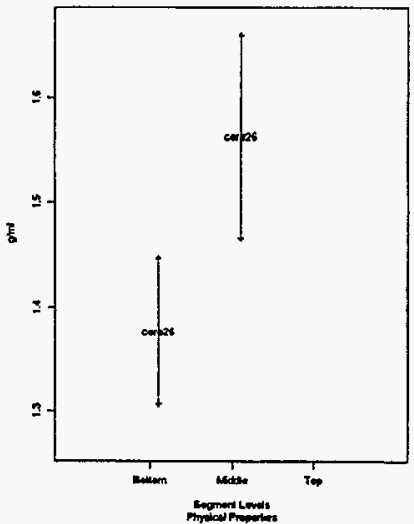

(41)

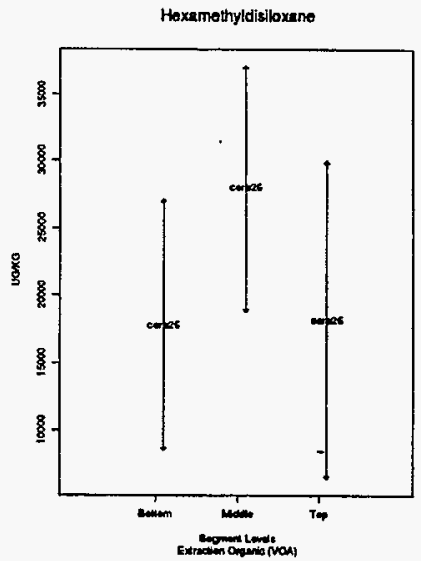

(43)

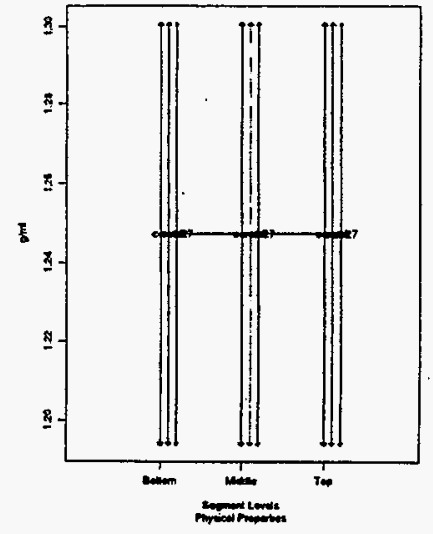

(42)

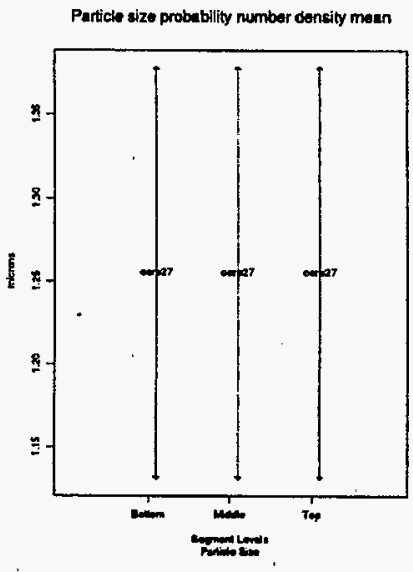

(44) 
Figure C-1. Plots for Best Method of Analysis. (page 12 of 14)

Particle sizo probability volume density mean

Settied solids

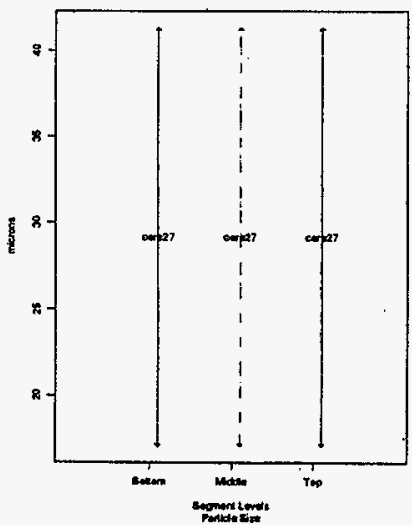

(45)

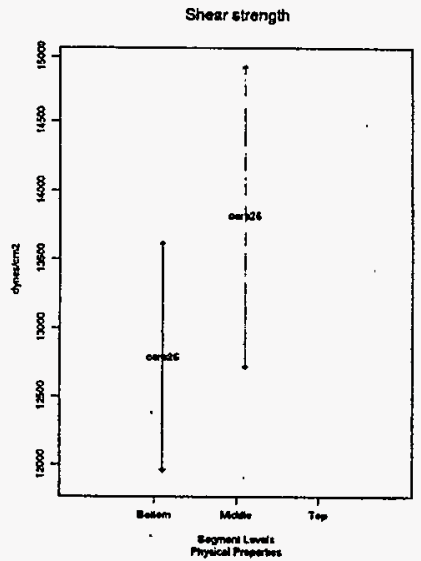

(47)

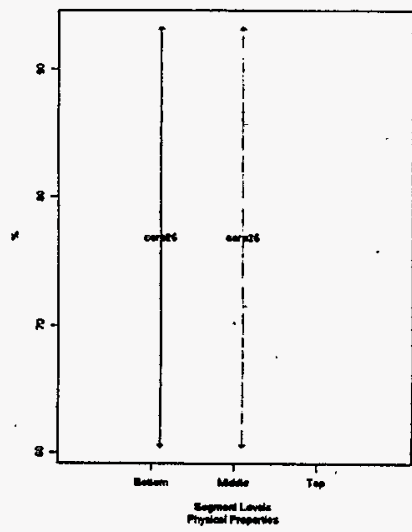

(46)

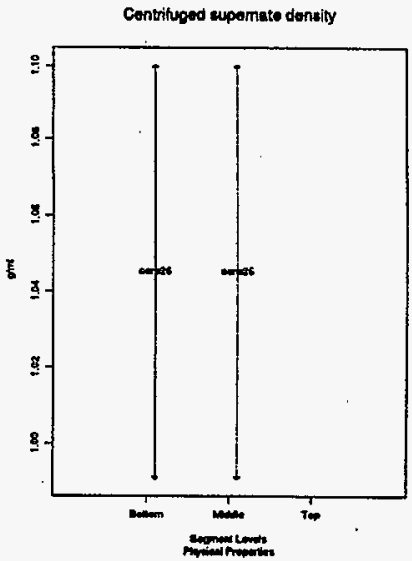

(48) 
Figure C-1. Plots for Best Method of Analysis. (page 13 of 14)

Toluene

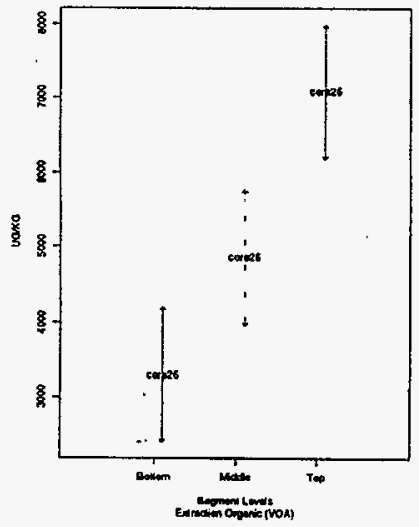

(49)

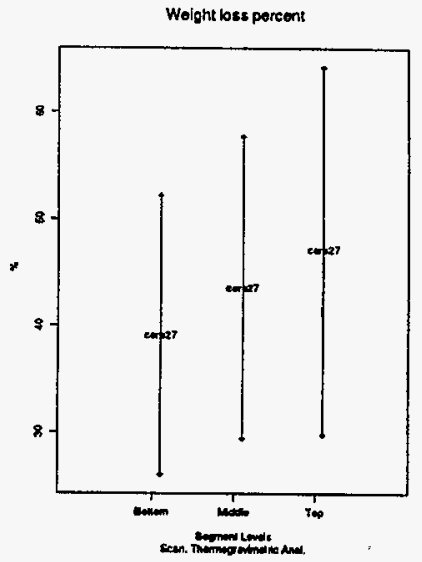

(51)
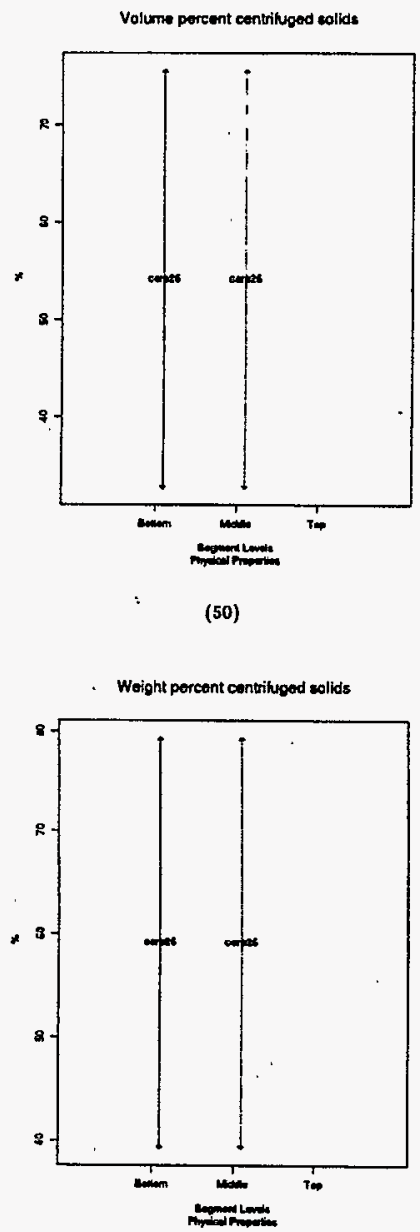

(52) 
Figure C-1. Plots for Best Method of Analysis. (page 14 of 14)

Weight percent solids

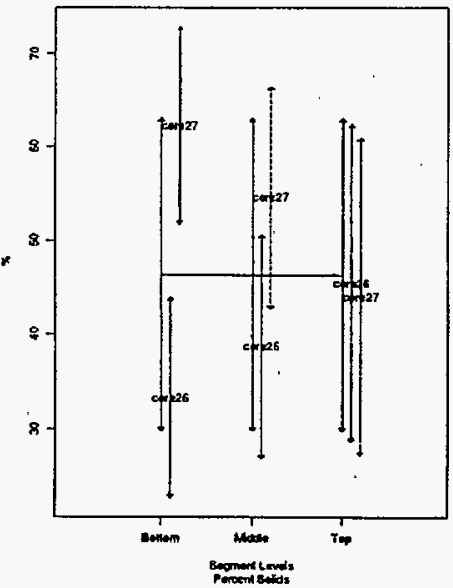

(53)
Yiold point

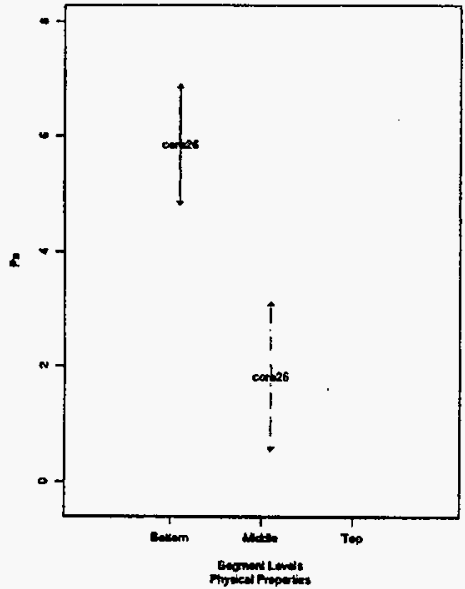

(54) 
WHC-SD-WM-ER-550 Rev 0

This page intentionally left blank. 
WHC-SD-WM-ER-550 Rev 0

APPENDIX D

RAW DATA SET SUMMARY

D-1 
This page intentionally left blank. 


\section{APPENDIX D}

\section{RAW DATA SET SUMMARY}

This appendix describes the format of the B-201 data set used to produce the results discussed in this report. The data set contains chemical measurements made by the 325-A Laboratory on B-201 core samples. The data was originally downloaded from the Tank Characterization Database (TCD). The following changes were made to the data set in preparation for the various statistical analyses:

1. The $\mathrm{Na}_{2} \mathrm{O}_{2}$ Fusion ICP analyses were removed for all constituents with the exception of nickel and potassium.

2. The KOH Fusion ICP analyses were removed from the data set for nickel and potassium.

3. Only 17 of the original $40 \mathrm{TCD}$ fields remain in the data set. The 17 fields remaining in the data set are those that are pertinent to the data analysis.

4. Any sample result that was below the detection limit was replaced with the detection limit value if it was available.

5. All of the TCLP results from the Acid Digestion ICP analysis method were removed to avoid confusion with the standard Acid Digestion ICP analyses.

6. The blanks were subtracted from all constituent sample results for the ICP Fusion analysis method.

7. The organics results were converted from parts per billion to parts per million.

An electronic ASCII copy of the B-201 data set is available upon request. This data set does not include any of the QA data (i.e., matrix spikes and method blanks). The B-201 data set is 5,109 records in length. Table D-1 describes the contents of each field. Reference DOE 1994 contains more information on the format of the data in the TCD. 
Table D-1. Description of B-201 Data Set Fields.

\begin{tabular}{|c|l|}
\hline Field & \multicolumn{1}{|c|}{ Description } \\
\hline 1 & Core Number \\
\hline 2 & Segment or Composite Number \\
\hline 3 & Analytical Method Name \\
\hline 4 & Phase of the Waste Sample (i.e., Solid or Liquid) \\
\hline 5 & Sample Location (TOP and BOTTOM are homogenization samples and TOTAL is the standard sample) \\
\hline 6 & Sample ID Number (assigned by the 325-A Laboratory) \\
\hline 7 & Dilution Factor \\
\hline 8 & Sample Batch Number \\
\hline 9 & Table and Page Number in the Validation Report that contains the sample results \\
\hline 10 & Constituent name \\
\hline 11 & Measured Sample Result \\
\hline 12 & Result Type (e.g., Primary Result, Duplicate Result) \\
\hline 13 & Result Units \\
\hline 14 & Detection Limit \\
\hline 15 & Detection Limit Units \\
\hline 16 & Data Quality Flags assigned by Hanford Analytical Services (HAS) \\
\hline 17 & Field indicating if a result is above the detection limit (T = above DL, F = below DL) \\
\hline
\end{tabular}

Table D-2 contains an example of three records from the B-201 dataset.

Table D-2. Excerpt from B-201 Raw Data Set.

\begin{tabular}{|c|c|c|c|c|}
\hline Field 1 & Field 2 & Field 3 & Field 4 & Field 5 \\
\hline Field 6 & Field 7 & Ficld 8 & Field 9 & Field 10 \\
\hline Field 11 & Field 12 & Field 13 & Field 14 & Field 15 \\
\hline Field 16 & Field 17 & & & \\
\hline core26 & 3 & Extraction Organic (VOA) & s & TOTAL \\
\hline BLANK & 1.0 & & PG.145 & Tetrachloroethane \\
\hline $3.800000 \mathrm{e}+06$ & PRIMARY_RESULT & UG/G & NA & \\
\hline UDR & $\mathbf{F}$ & & & \\
\hline core 26 & 3 & Acid Digestion ICP & $\mathbf{S}$ & TOP \\
\hline $9203238 \mathrm{~A}$ & 10.0 & 21 & $\mathrm{Pg} 67$, Table 2-2e & Tellurium \\
\hline $2.087700 e+02$ & DUPLICATE RESULT & $\mathrm{UG} / \mathrm{G}$ & 208.77000 & $\mathbf{U G} / \mathbf{G}$ \\
\hline $\mathbf{U}$ & $\mathbf{F}$ & & & \\
\hline core 27 & Compl & Fusion ICP & $\mathbf{S}$ & ВOTTOM \\
\hline $9210669 \mathrm{H} 1 \mathrm{~B}$ & 2.0 & 49 & Pg 353, Table 2-lb & Tellurium \\
\hline $4.293200 e+02$ & DUPLICATE RESULT & $\mathrm{UG} / \mathrm{G}$ & 429.32000 & UG/G \\
\hline $\mathrm{U}$ & $\mathrm{F}$ & & & \\
\hline
\end{tabular}


WHC-SD-WM-ER-550 Rev 0

APPENDIX E

RHEOLOGICAL PROPERTIES TABLES AND PLOTS

E-1 
WHC-SD-WM-ER-550 Rev 0

This page intentionally left blank. 


\section{APPENDIX E}

\section{RHEOLOGICAL PROPERTIES TABLES AND PLOTS}

This appendix contains an excerpt from Shaver 1993 on the rheological properties measured on tank B-201, core 26 sample. Shear strength, waste flow properties, and settling behavior are discussed and tables and plots are given.

The shear strength of the three segment samples was measured in at least duplicate. The average values for the shear strength of segments 2, 5, and 8 are reported in Table 5-5.

Shear stress as a function of shear rate was run in duplicate at ambient temperature for each of the segment materials. The shear stress exceeded the maximum value of system being used for this measurement ( $8500 \mathrm{~Pa}$ or $\left.85.000 \mathrm{dynes} / \mathrm{cm}^{2}\right)$. The cone was being rotated at a significantly larger rate than was used for the shear strength measurement; therefore, the shear stress measured would be higher than the shear strength of the material. Some drying of the sample may also have occurred on the plate, causing the shear strength to be higher than expected. Due to drying of the samples at $95^{\circ} \mathrm{C}\left(203^{\circ} \mathrm{F}\right)$, the shear stress of the samples as a function of shear rate could not be measured on the as-received samples or 1:1 dilution at this temperature.

Rheological properties were measured in duplicate on the dilutions of segments 2,5 , and 8 at ambient temperature. A single measurement of shear stress as a function of shear rate was run at a 3:1 dilution at $95{ }^{\circ} \mathrm{C}$. Only one measurement was made because the sample was drying too quickly to make a duplicate measurement which accurately represented the viscosity of the sample.

The 1:1 dilution samples exhibit yield psuedoplastic behavior, and the data was fit to a yield power law expression. The equation to fit this data is given by Equation 6 . The parameters from this fit were input into a model developed by Hanks to predict the flow properties for non-Newtonian fluids. The data-obtained from this model is PNNL QA Impact Level III. The data obtained includes critical velocity for turbulent flow and critical Reynolds number. This data and the fit parameters for each sample are given in Tables E-1 and $\mathrm{E}-2$.

$$
\tau=\tau_{y}+K \gamma^{n}
$$


where:

$\begin{array}{rll}\tau & =\text { Shear stress } \\ \tau_{\mathrm{y}} & =\text { Yield point } \\ K & =\text { Consistency parameter } \\ \gamma & =\text { Shear rate } \\ n & =\text { Flow behavior index. }\end{array}$

Table E-1. Power Law Curve Fit Parameters of 1:1 Dilution Samples.

\begin{tabular}{|c|c|c|c|r|r|}
\hline Segment & Temp. $\left({ }^{\circ} \mathbf{C}\right)$ & Run & $\begin{array}{c}\text { Yield } \\
\text { Point } \\
\text { (Pa) }\end{array}$ & $\begin{array}{c}\text { Consistency } \\
\text { Factor } \\
\text { (Pa sec) }\end{array}$ & $\begin{array}{c}\text { Flow } \\
\text { Behavior } \\
\text { Index }\end{array}$ \\
\hline 2 & 30 & 1 & 1.16 & $6.51 \mathrm{e}-03$ & 0.92 \\
& & 2 & 2.29 & $1.61 \mathrm{e}-02$ & 0.81 \\
\hline 5 & 30 & 2 & 4.77 & $8.89 \mathrm{e}-03$ & 1.00 \\
& & 3 & 6.38 & $2.34 \mathrm{e}-02$ & 0.85 \\
\hline 8 & 29 & 1 & 9.53 & $2.82 \mathrm{e}-02$ & 0.86 \\
& & 2 & 6.60 & $6.66 \mathrm{e}-02$ & 0.73 \\
\hline
\end{tabular}

Table E-2. Flow Properties of 1:1 Dilution Samples.

\begin{tabular}{|c|c|c|c|r|r|r|}
\hline Segment & $\begin{array}{c}\text { Temp } \\
\left({ }^{\circ} \mathbf{C}\right)\end{array}$ & Run & $\begin{array}{c}\text { Pipe } \\
\text { (in.) }\end{array}$ & $\begin{array}{c}\text { Critical } \\
\text { Velocity } \\
\text { (ft/sec) }\end{array}$ & $\begin{array}{c}\text { Critical } \\
\text { Flow } \\
\text { Rate } \\
\text { (gal/min) }\end{array}$ & $\begin{array}{c}\text { Critical } \\
\text { Reynolds } \\
\text { Number }\end{array}$ \\
\hline 2 & 30 & 1 & 2 & 1.9 & 19 & 8,500 \\
& & 2 & 3 & 1.7 & 38 & 10,800 \\
& & 2 & 2.6 & 27 & 8,100 \\
\hline 5 & 30 & 1 & 2 & 2.3 & 54 & 9,900 \\
& & & 3 & 4.1 & 43 & 8,600 \\
& & 2 & 2 & 3.6 & 84 & 11,300 \\
& & & 3 & 4.7 & 49 & 8,100 \\
\hline 8 & 30 & 1 & 2 & 5.2 & 97 & 10,000 \\
\hline & & & 3 & 5.9 & 61 & 8,000 \\
& & 2 & 2 & 5.3 & 122 & 9,900 \\
& & & 3 & 4.9 & 113 & 7,200 \\
\hline
\end{tabular}


The 3:1 dilutions aiso exhibit yield psuedoplastic behavior with a yield point of less than $0.5 \mathrm{~Pa}$. The viscosity and yield point of the samples was so low that no attempt was made to model the data. At shear rates greater than $100 \mathrm{~s}^{-1}$, the viscosity of the $3: 1$ dilution samples was less than $5 \mathrm{cP}$. The viscosity of the $3: 1$ dilution decreases significantly with increasing temperature.

Plots of shear stress and viscosity as a function of shear rate for the dilutions are shown in Figures E-1, E-2, and E-3.

Figure E-1. Settling Behavior of Segment 2 (Core 26).

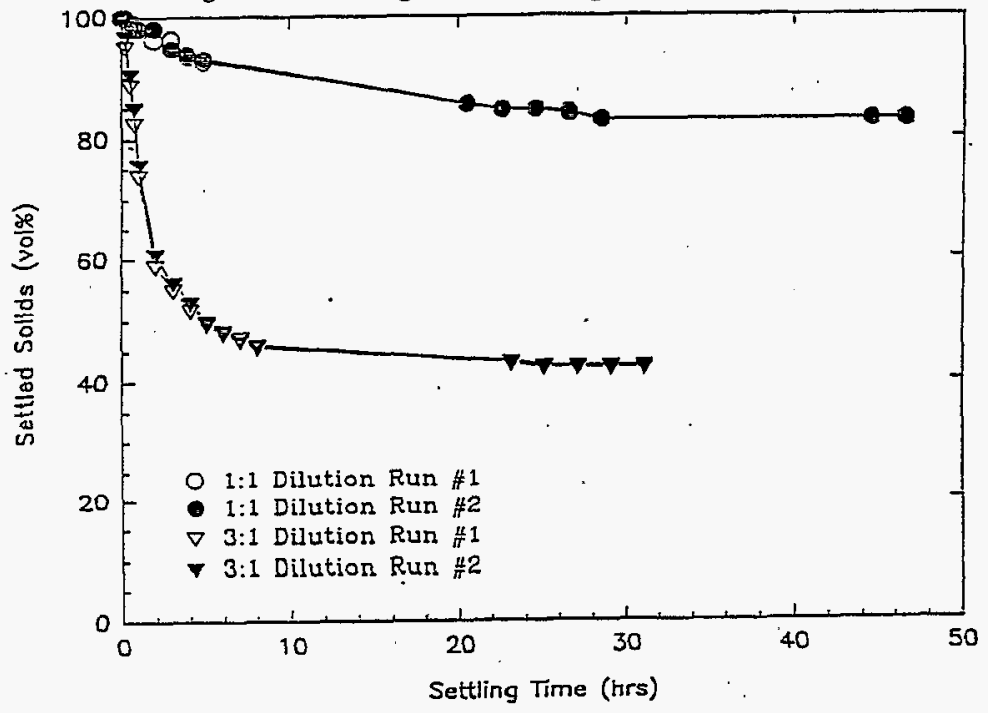


Figure E-2. Settling Behavior of Segment 5 (Core 26).

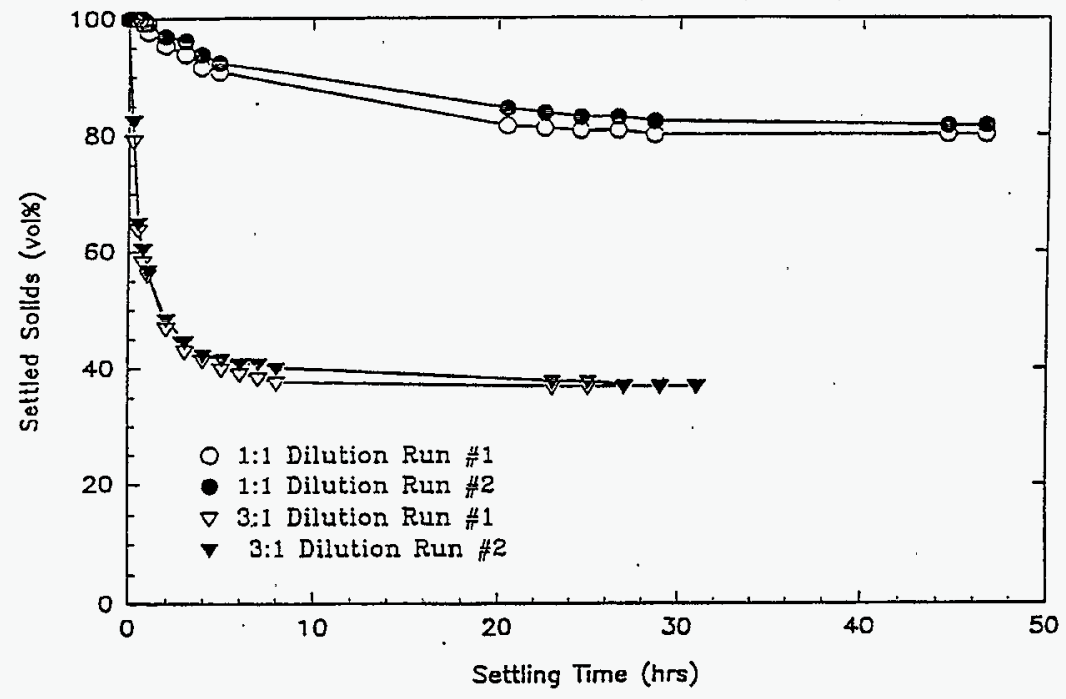


WHC-SD-WM-ER-550 Rev 0

Figure E-3. Settling Behavior of Segment 8 (Core 26).

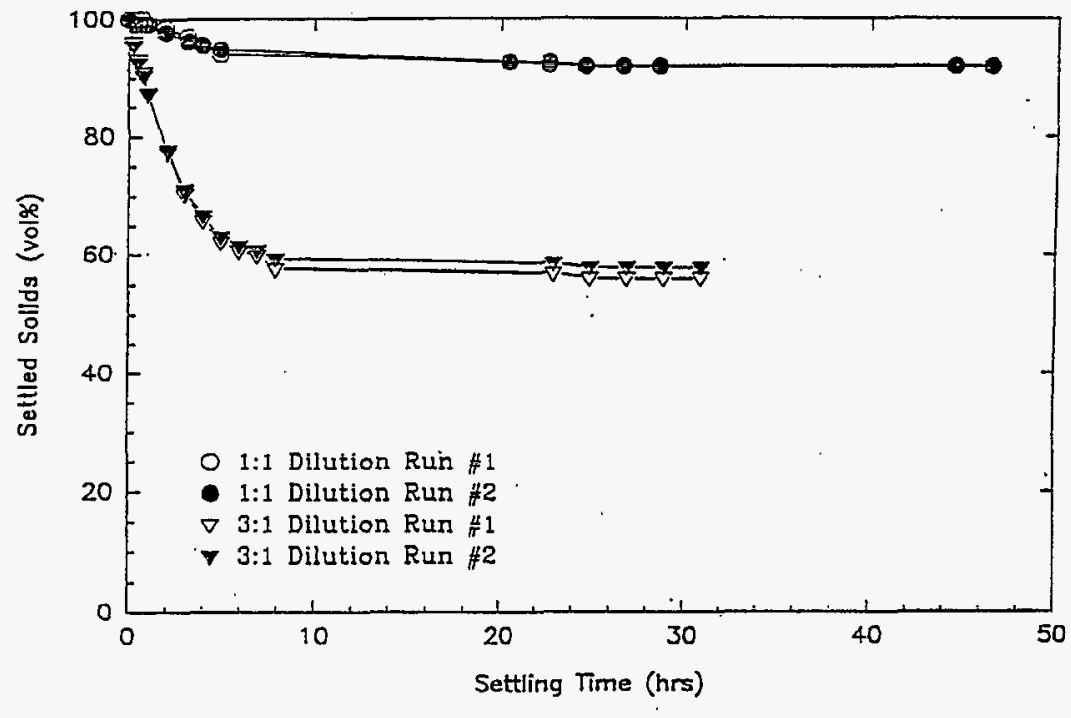


WHC-SD-WM-ER-550 Rev 0

This page intentionally left blank. 


\section{DISTRIBUTION SHEET}

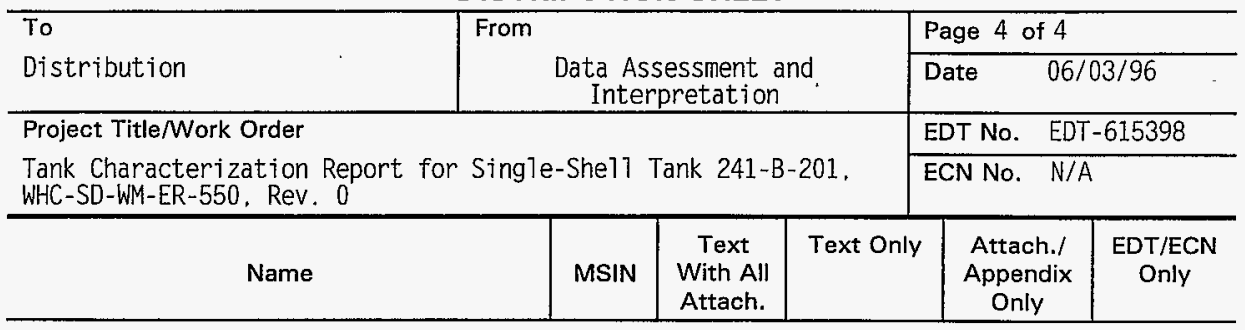

Westinghouse Hanford Company continued

W. C. Miller

C. T: Narquis

D. E. Place

D. A. Reynolds

L. M. Sasaki (2)

L. W. Shelton, Jr.

B. C. Simpson

G. L. Troyer

M. S. Waters

L. R. Webb

K. A. White

FIC (Fank Fam Information Center)

Central Files (D) plus creiginal

ERC (Environmental Resource Center) OSTI (2)

TCRC (10)

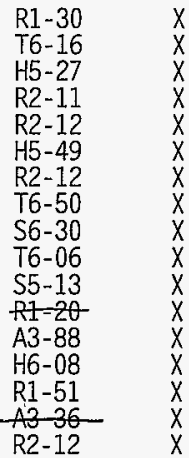




\section{DISTRIBUTION SHEET}

\begin{tabular}{|c|c|c|c|c|c|}
\hline \multirow{2}{*}{$\begin{array}{l}\text { To } \\
\text { Distribution }\end{array}$} & \multirow{2}{*}{\multicolumn{3}{|c|}{$\begin{array}{l}\text { Data Assessment a } \\
\text { Interpretation }\end{array}$}} & \multicolumn{2}{|l|}{ Page 3 of 4} \\
\hline & & & & \multicolumn{2}{|c|}{$06 / 03 / 96$} \\
\hline \multicolumn{6}{|l|}{ Project Title/Work Order } \\
\hline \multicolumn{4}{|c|}{$\begin{array}{l}\text { Tank Characterization Report for Single-She11 Tank 241-B-201. } \\
\text { WHC-SD-WM-ER-550, Rev. } 0\end{array}$} & ECN No. $\mathrm{N} /$ & \\
\hline Name & MSIN & $\begin{array}{c}\text { Text } \\
\text { With All } \\
\text { Attach. }\end{array}$ & Text Only & $\begin{array}{l}\text { Attach./ } \\
\text { Appendix } \\
\text { Only }\end{array}$ & $\begin{array}{l}\text { EDT/ECN } \\
\text { Only }\end{array}$ \\
\hline
\end{tabular}

ONSITE

Department of Ecology
A. B. Stone
B5-18
$x$

Department of Eneray - Richland Operations

J. F. Thompson

W. S. Liou

$57-54$

$57-54$

N. W. WiTlis

$57-54$

$x$
$x$
$x$

ICF-Kaiser Hanford Company

R. L. Newe1l

S3-09

$x$

Pacific Northwest Laboratory

N. G. Colton

J. R. Gormsen

S. A. Hartley

J. G. Hi 11

G. J. Lumetta

A. F. Noonan

K3-75

K $7-28$

$\mathrm{K} 5-12$

K7-94

P7-25

K9-81

Westinghouse Hanford Company

H. Babad

D. A. Barnes

G. R. Bloom

J. M. Conner

W. L. Cowley

G. L. Dunford

E. J. Eberlein

D. B. Engelman

J. S. Garfield

J. D. Guberski

D. L. Herting

G. Jansen

G. D. Johnson

T. J. KeTley

N. W. Kirch

M. J. Kupfer

J. E. Meacham

S7-14

R1-80

H5-61

R2-12

A3-37

S7-81

R2-12

RI- 49

H5- 49

R2-06

T6-09

H6-33

S7-15

S7-21

R2-11

H5- 49

S7-15

$X$
$X$
$X$
$X$
$X$ 


\section{DISTRIBUTION SHEET}

\begin{tabular}{|c|c|c|c|c|c|c|}
\hline To & \multirow{2}{*}{\multicolumn{3}{|c|}{$\begin{array}{l}\text { From } \\
\text { Data Assessment and } \\
\text { Interpretation }\end{array}$}} & \multicolumn{3}{|c|}{ Page 2 of 4} \\
\hline Distribution & & & & Date & \multicolumn{2}{|c|}{$06 / 03 / 96$} \\
\hline \multicolumn{4}{|c|}{ Project Title/Work Order } & \multicolumn{3}{|c|}{ EDT No. EDT-615398 } \\
\hline \multicolumn{4}{|c|}{$\begin{array}{l}\text { Tank Characterization Report for Single-She11 Tank 241-B-201, } \\
\text { WHC-SD-WM-ER-550, Rev. } 0\end{array}$} & \multicolumn{3}{|c|}{ ECN No. $\quad \mathrm{N} / \mathrm{A}$} \\
\hline Name & MSIN & $\begin{array}{l}\text { Text } \\
\text { With All } \\
\text { Attach. }\end{array}$ & Text Only & $\begin{array}{l}\text { Atte } \\
\text { App } \\
\text { O }\end{array}$ & & $\begin{array}{c}\text { EDT/ECN } \\
\text { Only }\end{array}$ \\
\hline
\end{tabular}

SAIC

20300 Century Boulevard, Suite 200-B

Germantown, MD 20874

H. Sutter

$X$

555 Quince Orchard Rd., Suite 500

Gaithersburg. MD, 20878

P. Szerszen

$x$

Los Alamos Laboratory

CST-14 MS-J586

P. 0. Box 1663

Los Alamos, NM 87545

S. F. Agnew (4)

$X$

Los Alamos Technical Associates

T. T. Tran

B1-44 $x$

Ogden Environmenta]

101 East Wel Isian Way

Richland. WA 99352

R. J. Anema

$x$

CH2M Hill

P. 0. BoX 91500

Bellevue, WA 98009-2050

M. McAfee

$X$

Tank Advisory Pane]

102 Windham Road

Oak Ridge, TN 37830

D. O. Campbe17 


\section{DISTRIBUTION SHEET}

\begin{tabular}{|c|c|c|c|c|c|}
\hline To & \multirow{2}{*}{\multicolumn{3}{|c|}{$\begin{array}{l}\text { From } \\
\text { Data Assessment and } \\
\text { Interpretation }\end{array}$}} & \multicolumn{2}{|l|}{ Page 1 of 4} \\
\hline Distribution & & & & Date & $06 / 03 / 96$ \\
\hline \multicolumn{4}{|c|}{ Project Title/Work Order } & \multicolumn{2}{|c|}{ EDT No. EDT-615398 } \\
\hline \multicolumn{4}{|c|}{$\begin{array}{l}\text { Tank Characterization Report for Single-Shel1 Tank 241-B-201, } \\
\text { WHC-SD-WM-ER-550, Rev. } 0\end{array}$} & \multicolumn{2}{|c|}{ ECN No. N/A } \\
\hline Name & MSIN & $\begin{array}{l}\text { Text } \\
\text { With All } \\
\text { Attach. }\end{array}$ & Text Only & $\begin{array}{l}\text { Attach./ } \\
\text { Appendix } \\
\text { Only }\end{array}$ & $\begin{array}{c}\text { EDT/ECN } \\
\text { Only }\end{array}$ \\
\hline
\end{tabular}

\section{OFFSITE}

Sandia National Laboratory

P.0. Box 5800

MS-0744, Dept. 6404

Atbuquerque, NM 87815

D. Powers

$x$

Nuclear Consulting Services Inc.

P. 0. Box 29151

Columbus, OH 43229-01051

J. L. Kovach

$x$

Chemical Reaction Sub-TAP

P.0. Box 271

Lindsborg, KS 67456

B. C. Hudson

$x$

Tank Characterization Panel

Senior Technical Consultant

Contech

7309 Indian School Road

Albuquerque, NM 87110

J. Arvisu

$x$

U. S. Department of Energy - Headquarters

Office of Environmenta? Restoration and Waste Management EM-563 12800 Midd7ebrook Road

Germantown, MD 20874

J. A. Poppitti

$X$

Jacobs Engineering Group

3250 W. CTearwater

Kennewick, WA 99336 